\section{SNF Project \\ 5. Proj/Prog/Dopt/Div.: \\ SNF $W-441$}

2. To: (Recolving Organization)

\section{Orighator Remarka:}

Transmittal of SNE-5331, "ARROW (Version 2) - Commercial Software Validation and Configuration Control"

\section{Recolver Remarks:}

11A. Dedign Baceline Document? O Yes

\section{Related EDT No.: \\ $\mathrm{N} / \mathrm{A}$ \\ 7. Purchase Order No.: \\ N/A \\ 9. Equlp./Component No.: \\ N/A}

10. Syatem/Bldg J Faclity:

CVD 142K

12. Major Aaem. Dwg. No.

N/A

13. Pormily/Pormil Application No.:

$\mathrm{N} / \mathrm{A}$

14. Required Respones Date:

N/A

\begin{tabular}{|c|c|c|c|}
\hline (F) & (G) & (H) & (I) \\
\hline $\begin{array}{l}\text { pproval } \\
\text { nato } \\
\text { nator }\end{array}$ & $\begin{array}{l}\text { Reseor } \\
\text { or Tran } \\
\text { mitial }\end{array}$ & $\begin{array}{l}\text { Orlot } \\
\text { nator } \\
\text { Dispo- } \\
\text { stion }\end{array}$ & $\begin{array}{l}\text { Recolv- } \\
\text { of } \\
\text { Difpo- } \\
\text { eition }\end{array}$ \\
\hline
\end{tabular}

15

DATA TRANSMITTED

\begin{tabular}{|l|l|}
\hline $\begin{array}{l}\text { (A) } \\
\text { lim. } \\
\text { No. }\end{array}$ & (B) Document/Drawing No. \\
\hline 1 & SNF-5331 \\
\hline & \\
\hline & \\
\hline & \\
\hline & \\
\hline & \\
\hline
\end{tabular}

16.

\begin{tabular}{c|c|l}
$\begin{array}{c}\text { (C) Sheet } \\
\text { No. }\end{array}$ & (D) Rov. & (E) Tlite or Description of Date Tranamitted \\
\hline
\end{tabular}

\begin{tabular}{|l|l|}
\hline all & 0 \\
\hline & \\
\hline & \\
\hline & \\
\hline & \\
\hline
\end{tabular}

ARROW (Version 2)

SQN Commercial Software

Validation configuration Control

\begin{tabular}{|c|c|c|c|c|}
\hline Approval Decienctor (F) & \multicolumn{2}{|c|}{ Reason for Tranamital (G) } & \multicolumn{2}{|c|}{ Disposition (H) \& (I) } \\
\hline 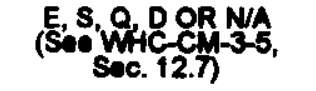 & $\begin{array}{l}\text { 1. Approval } \\
\text { 2. Relacea } \\
\text { 3. Iniomation }\end{array}$ & $\begin{array}{l}\text { 4. Reviow } \\
\text { 5. Podt-Roviow } \\
\text { 6. Dint. (Recelpt Acknow. Required) }\end{array}$ & $\begin{array}{l}\text { 1. Approved } \\
\text { 2. Approved w/comment } \\
\text { 3. Deapproved w/comment }\end{array}$ & $\begin{array}{l}\text { 4. Reviowed no/comment } \\
\text { 5. Revlewed w/comment } \\
\text { 8. Receipt scknowledged }\end{array}$ \\
\hline
\end{tabular}
17. (See Approvil Deaignator for required angetures)

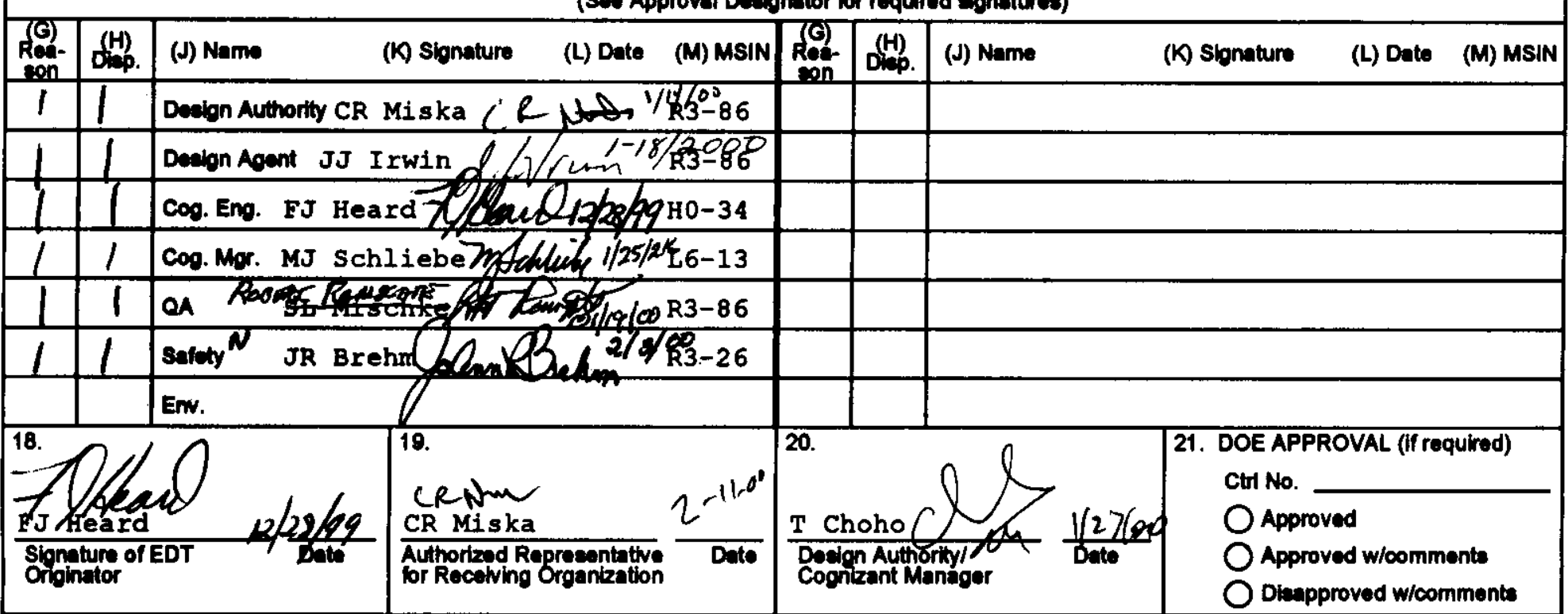




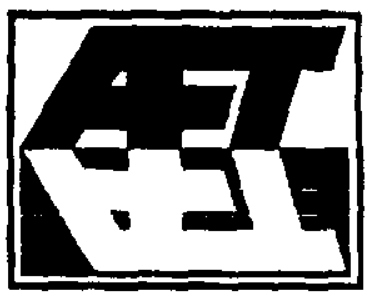

\section{Applied Flow Technology}

Ship: 400 W. Highway 24, Suite 201, Woodland Park, CO 80863 USA Mail: P.O. Box 6358, Woodland Park, CO 80866-6358 USA (719) 686-1000/ FAX (719) 686-1001 INTERNET: www.aft.com, info@aft.com

\section{Facsimile Cover Sheet}

\begin{tabular}{|l|l|l|l|}
\hline To: & Fred Heard & From: & Tom Glassen \\
\hline Company: & Fluor Hanford & & \\
\hline Fax: & $609-372-3655$ & Fax: & (719) 686-1001 \\
\hline Phone: & $509-376-6562$ & Phone: & (719) 686-1000 \\
\hline Pages: & 3 & Date: & Jan. 6, 2000 \\
\hline
\end{tabular}

Subj: Arrow Verification

Dear Fred:

I'm pleased to return the signed "Permission to use Copyrighted Material" form as well as page 8-13 applicable to example 8.14 for verification model 10 .

Best regards,

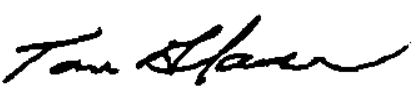

Tom Glassen

Director of Operations \&

Business Development 
Jañ-06-OO 12:10P App 1 1ed Flow Technology

$719686 \quad 1001$

P. 02 U1,utuvi 12:51 FDP

\section{EDT $-627034 p_{S} \cdot 3 / 3$}

\section{APPLICATION FOR PERMISSION TO USE YOUR COPYRICHTED MATERIAL}

To: Tom Glassen, Apelied Flow Technology

Date: January \& 2000

R.0. Box 6358

Nuodiluwn Park, CO B0868-6358

Pormission is requestod to reproduce the following copyrighted material from:

Problem description and result surmary for verlfication provloris 1 through 21 . Supject documents are supplied in electronic form with filenanes: verifyl doc through

verify21.doc. Subtect documents will be printed and attached as a supporting appendix.

Solections from toxt (specify by date of lasue, page. paragraph, or illustration; if desired, attach a copy of the material in question):

Thle of work or project in which this matertal will be included:

ARsoh (Veraion 2) - Conmercial software validation and contrguration control

Estimated publication date: January 2000

Author: E. J. Heard

Publisher (f applicable): Fruer hanferel, inc,

If the copyrighted material is not to be used in a published work, please provide s brief tescription of how $h$ is to be used:

\section{A selfaddressed stamped envelope is enclosed for your reply.}

Name: Prederick $I$ Heard

MSIN: HO-34

Date: January 04, 2000

Shnature:
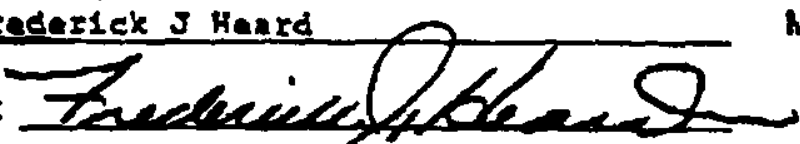

Creath the (fi required):

Applieation approved by:

Name: Thomas Glassen

Company Name: Apelred Flow Techollogy

Signatiurs:

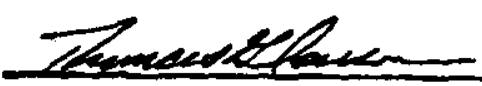

Date: $\angle t=2000$

Sirector of Qperetione

(on behatr os 


\title{
ARROW (Version 2) - Commercial Software Validation and Configuration Control
}

\author{
r. J. Heard \\ Fluor Daniel Hanford, Ire. $m$ \\ Richland, WA 99352 \\ U.S. Department of Energy Contract DE-AC08-98RL13200
}

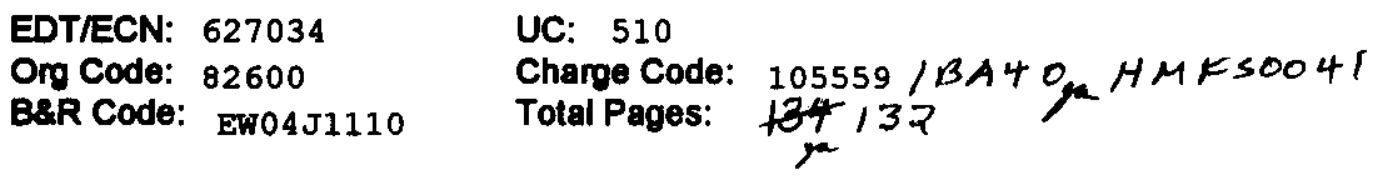

Key Words: software validation, configuration control, computer program flow network, piping, compressible, ARROW, Applied Flow Technology

\begin{abstract}
ARROW (Version 2), a compressible flow piping network modeling and analysis computer program from Applied Flow Technology, was installed for use at the U.S. Department of Energy Hanford Site near Richland, Washington. A series of sample problems were performed to validate the software and confirm proper installation. The sample problems are compared to published results.
\end{abstract}

TRADEMARK DISCLAIMER. Relerence horein to any epecilic commercial product, procees, or service by trade name, tredemerk, manufecturer, or otherwies, does not nocesearity conetitute or imply lis endoreement, recommendation, or fevoring by the United States Government or any agency thereof or the contractors or subcontractors.

Printed in the United States of America. To obtain coples of this document, contact: Document Control Services, P.O. Box 950, Mallotop H6-08, Richland WA 99352, Phono (500) 372-2420; Fax (509) 376-4989.
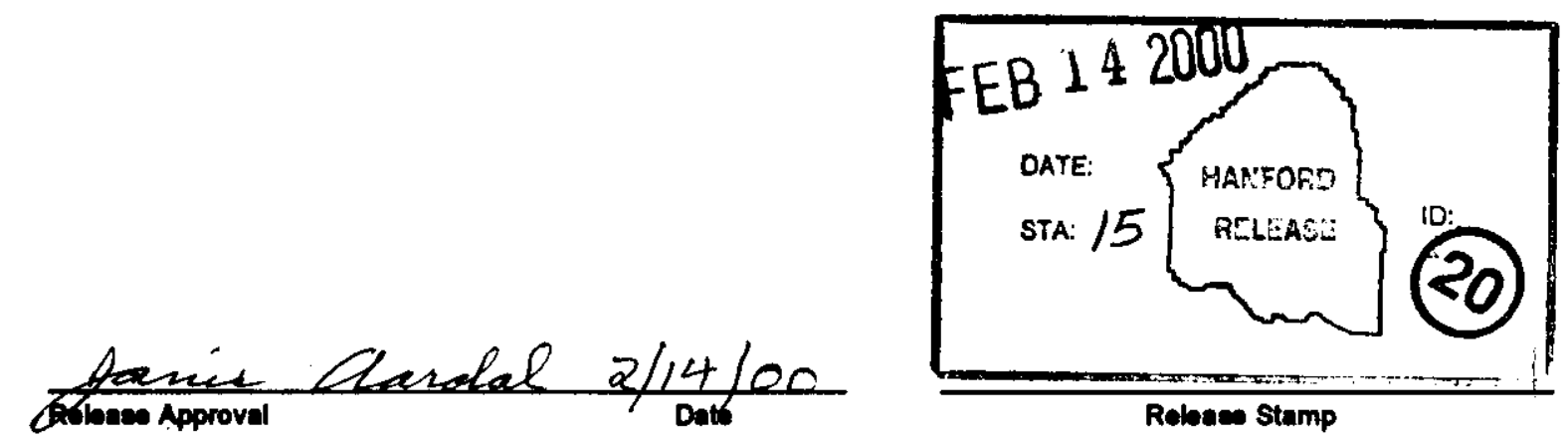

Approved For Public Release 
SNF-5331, Rev. 0

\section{ARROW (Version 2) - Commercial Software Validation and Configuration Control}

Prepared by:

F. J. Heard

Fluor Daniel Hanford, Inc.

December 1999 
SNF-5331, Rev. 0

\section{ABSTRACT}

ARROW (Version 2) a compressible flow piping network modeling and analysis program from Applied Flow Technology was installed for use at the Department of Energy (DOE) Hanford site near Richland, Washington. A series of tests were performed to validate the software and confirm proper installation. The sample problems are compared with published results. 


\section{TABBLE OF CONTENTS}

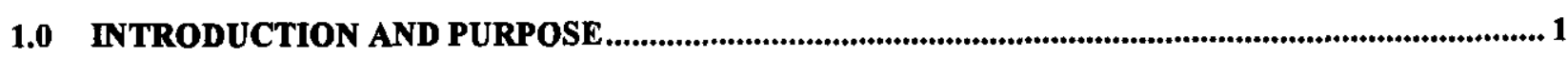

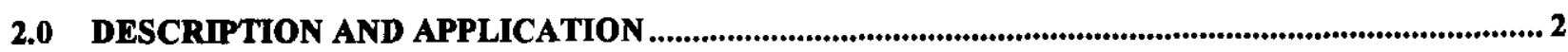

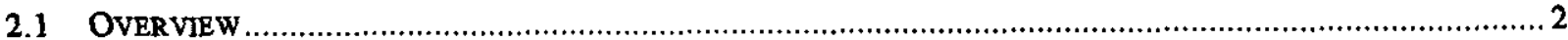

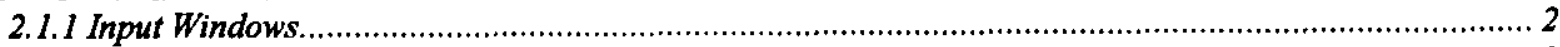

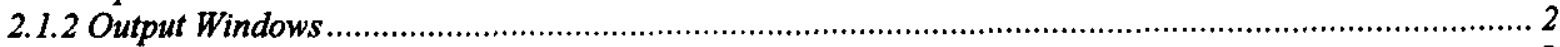

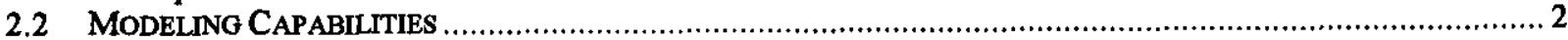

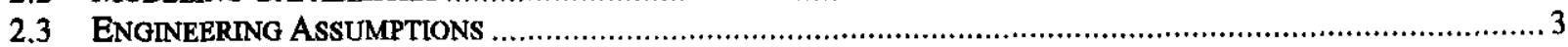

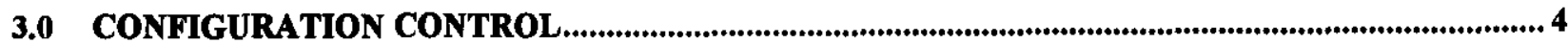

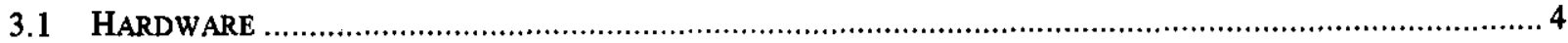

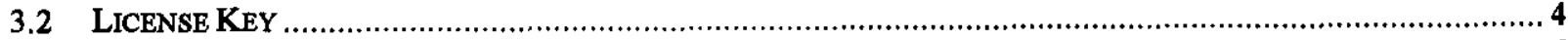

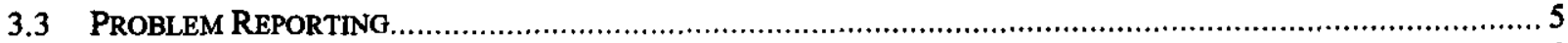

3.4 USER MANUAL

4.0 VALIDATION

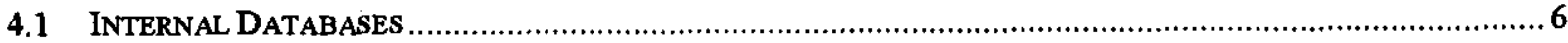

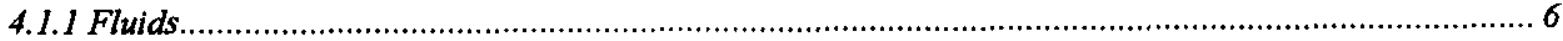

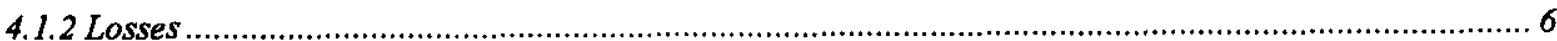

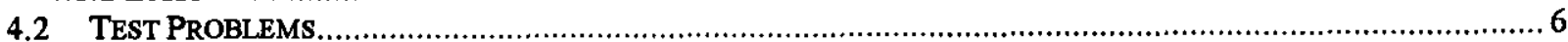

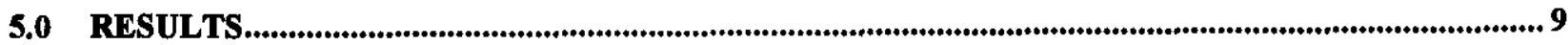

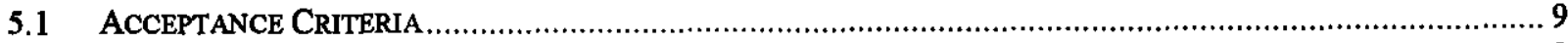

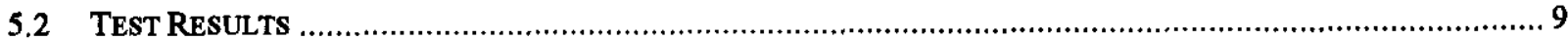

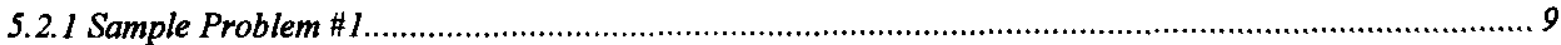

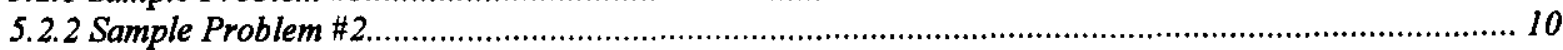

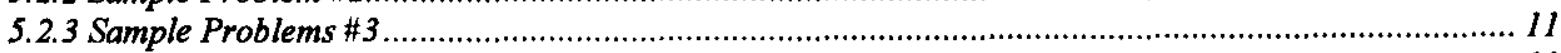

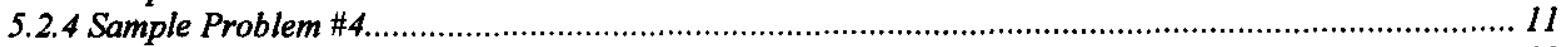

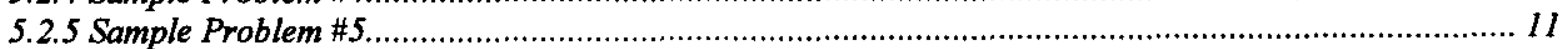

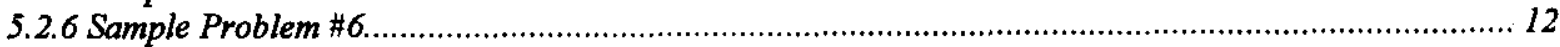

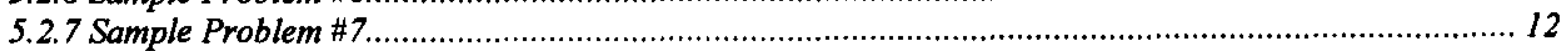

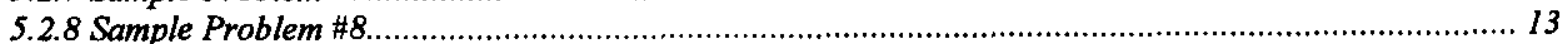

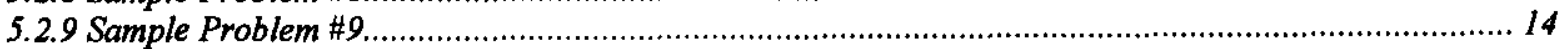

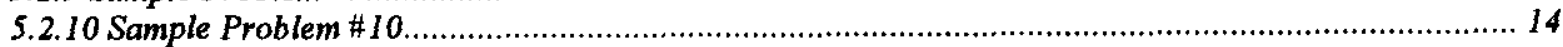

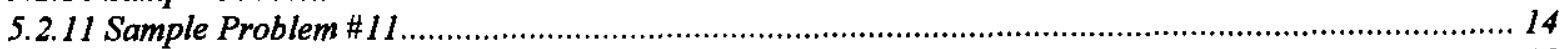

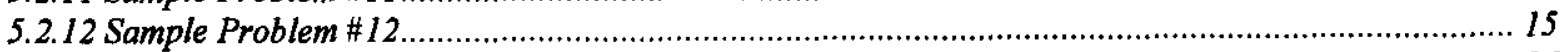

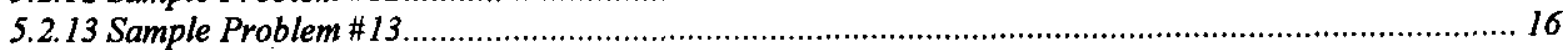

5.2.14 Sample Problem \#14

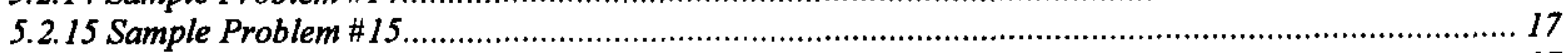

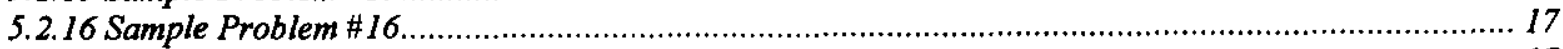

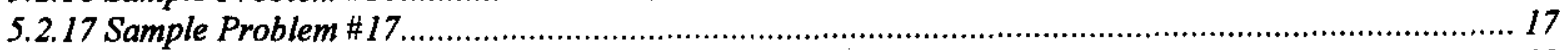

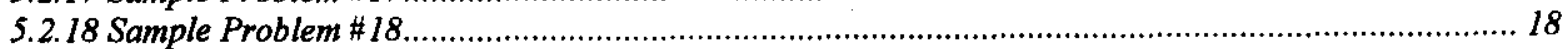

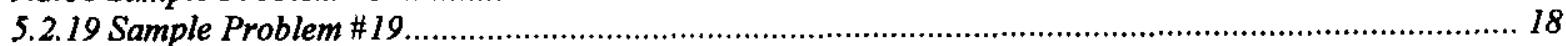

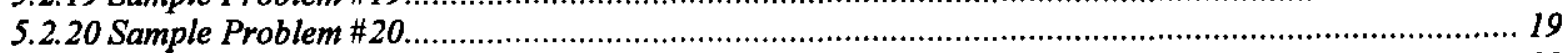

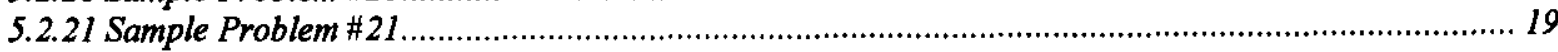

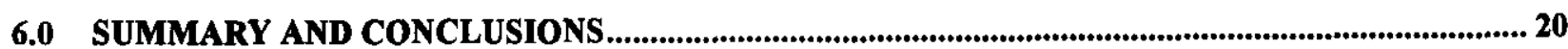

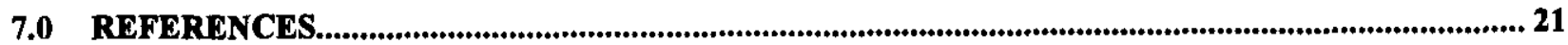

APPENDIX A - SAMPLE PROBLEM DESCRIPTIONS, INPUT, AND OUTPUT ....................................... 22 


\section{TABLES}

Table 3-1 Hardware and System Requirements......................................... 4

Table 4-1 Listing of Sample Problems Supplied with ARROW ............................... 7

Table 5-1 Comparison of Results for Sample Problem \#1 ................................. 10

Table 5-2 Comparison of Results for Sample Problem \#2 …............................. 10

Table 5-3 Comparison of Results for Sample Problem \#3 ................................ 11

Table 5-4 Comparison of Results for Sample Problem \#4 ................................ 11

Table 5-5 Comparison of Results for Sample Problem \#5 .............................. 12

Table 5-6 Comparison of Results for Sample Problem \#6 ................................ 12

Table 5-7 Comparison of Results for Sample Problem \#7 ................................ 13

Table 5-8 Comparison of Results for Sample Problem \#8 …........................... 13

Table 5-9 Comparison of Results for Sample Problem \#9 ................................... 14

Table 5-10 Comparison of Results for Sample Problem \#10 ................................ 14

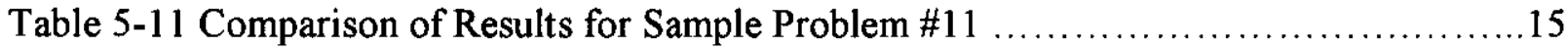

Table 5-12 Comparison of Results for Sample Problem \#12 …........................ 15

Table 5-13 Comparison of Results for Sample Problem \#13 ................................. 16

Table 5-14 Comparison of Results for Sample Problem \#14 ….......................... 16

Table 5-15 Comparison of Results for Sample Problem \#15 ............................. 17

Table 5-16 Comparison of Results for Sample Problem \#16 .............................17

Table 5-17 Comparison of Results for Sample Problem \#17 f............................ 18

Table 5-18 Comparison of Results for Sample Problem \#18 …......................... 18

Table 5-19 Comparison of Results for Sample Problem \#19 _............................ 18

Table 5-20 Comparison of Results for Sample Problem \#20 .............................. 19

Table 5-21 Comparison of Results for Sample Problem \#21 ............................. 19 


\subsection{INTRODUCTION AND PURPOSE}

The purpose of this report is to document the validation of AFT ARROW (Version 2) computer code consistent with the requirements of HNF-PRO-309 (Revision 0) "Computer Software Quality Assurance Requirements" for commercial-off-the-shelf (COTS) software.

AFT ARROW, hereafter referred to as ARROW, was supplied by Applied Flow Technology Corporation in Woodlawn Park, Colorado for use at the Department of Energy's Hanford site near Richland, Washington. "Applied Flow Technology", "AFT", and "AFT ARROW" are registered trademarks of the Applied Flow Technology Corporation.

Software validation is the process of evaluating a system or component during or at the end of the development process to determine whether it satisfies specified requirements. Validation involves running test cases to ensure that the computed output meets specified expectations and requirements, including numerical correctness of the results based on comparisons with alternate calculations (e.g., hand calculations, analytical solutions, or acceptance testing). 
SNF-5331, Rev. 0

\subsection{DESCRIPTION AND APPLICATION}

ARROW is piping network analysis tool for use with compressible flows. ARROW is intended as an aide for network analyses and not as a replacement for other design and analysis methods, including sound judgement, hand calculations, or independent checks. ARROW is supplied by Applied Flow Technology Corporation in Woodlawn Park, Colorado. "Applied Flow Technology", "AFT", and "AFT ARROW" are registered trademarks of the Applied Flow Technology Corporation.

\subsection{Overview}

ARROW uses a graphical interface that is based on drag-and-drop operations inherent to most Windows based applications. (Windows is a registered trademark of the Microsoft Corporation.) This allows the user to build a piping network using selections from a palette of components. Date is entered or edited for each of the components by double-clicking the component of interest. Additional global editing features are available from the menu or tool bars that simplify making large-scale changes tot he model.

ARROW has five integrated subordinate windows. The five windows can be viewed as an integrated environment for entering, processing, analyzing, and documenting the results of a piping network simulation. The user will work from one of the five windows at all times. Of the five windows, two are input windows and three are output windows

\subsubsection{Input Windows}

The two input windows are the Workspace window and the Model Data windows. These two windows, one graphical and the other text-based, process the input data for the model.

\subsubsection{Output Windows}

The three output windows are the Output, the Graph Results, and the Visual Report. The Output window is text-based. The Graph Results and Visual Report windows are graphical. These three windows are essential for reviewing the results of the analysis, spotting analysis errors, and preparing the results for documentation.

\subsection{Modeling Capabilities}

ARROW is a compressible steady-state one-dimensional Newtonian fluid network flow analysis program and can be used to model a variety of engineering systems including:

- Open and closed (recirculating) systems

- Network systems that branch or loop, with no limit on the number of loops

- Pressure driven systems

- Compressor or fan driven systems, including multiple compressors or fans in parallel and/or in series 
- Compressors or fans with variable speed, controlled discharge pressure and controlled flow

- Systems with pressure and/or flow control valves

- Systems with valves closed and compressors/fans turned off

- Systems with non-ideal gases

- Systems that experience sonic choking, including multiple sonic choke points

- Systems with non-reacting flow stream mixing and user defined mixtures (with optional Chempak)

- Systems with elevations changes or rotation such in turbomachinery

ARROW also provides hundreds of standard loss models based on well known references such as; Crane (1988), Idelchik (1994), and Miller (1990), but also allows the user to enter there own loss data. Variable or dynamic loss coefficients are supported. Variable loss coefficients may depend on flow, area ratios, and/or flow splits (such as tee's and wye's), and the angle of the connecting piping. ARROW also provides automatic calculation of friction factors using the well-known Colebrook-White correlation. Also provided is an extensive database of thermophysical properties for numerous fluids and gases

ARROW uses a Newton-Raphson method to solve the fundamental equations of pipe flow that govern conservation of mass, momentum, and energy. Solutions are obtained by iterative matrix methods that have been optimized for speed and convergence.

\subsection{Engineering Assumptions}

ARROW is based on the following fundamental fluid mechanics assumptions:

- Compressible flow

- All gases are superheated

- Steady-state conditions

- One-dimensional flow

- No chemical reactions

- Supersonic flow does not exist in the system 


\subsection{CONFIGURATION CONTROL}

ARROW was purchased from Applied Flow Technology located in Woodlawn, Colorado. ARROW is currently licensed for a single user on a single personal computer within a Windows 9x or NT environment. ARROW is considered commercial-off-the-shelf (COTS) software.

\subsection{Hardware}

ARROW was installed on an AST Bravo with a $200-\mathrm{MHz}$ Pentium processor and $128 \mathrm{MB}$ of main memory. The property tag is WC65744. The operating system is Windows 95 Version 4.00.950B. ARROW was installed by running the automated SETUP program located on the installation disk.

The ARROW installation program is very simple and standard for most Windows based applications. SETUP asks you where to unzip the program and where (i.e., pathname) to install the program. The executable files supplied with ARROW are complied code. No source code is supplied. SETUP handles all the file manipulations, short cuts, desktop icons, and registry entries required by Windows. ARROW was installed using the following default pathname: C: $\backslash$ ARROW.

To install and run AFT ARROW, the computer must meet the following minimum requirements:

Table 3-1. Hardware and System Requirements

\begin{tabular}{|l|l|l|}
\hline \multicolumn{1}{|c|}{ Feature } & \multicolumn{1}{|c|}{ Required } & \multicolumn{1}{c|}{ Recommended } \\
\hline IBM - Compatible Processor & 80486 & Pentium or higher \\
Hard Disk Space & $15 \mathrm{Mb}$ & \\
Floppy Drive & $31 / 2-$ Inch & \\
Monitor Resolution & VGA & SVGA or higher \\
Random Access Memory (RAM) & $32 \mathrm{Mb}$ & $48 \mathrm{Mb}$ \\
Mouse & Yes & \\
Math Co-processor & No & Yes \\
MS-Windows & Windows 95 or 98 & Windows NT \\
\hline
\end{tabular}

\subsection{License Key}

A license key is required to activate the AFT ARROW program for use. The license key is supplied by the vendor on a separate floppy disk. The user must supply the license key when requested during setup. The license key is copied from the floppy and stored within a hidden and encrypted file somewhere on the system. ARROW will check for the existence and content of the license file each time the program is activated.

The license key contains information pertaining to the registered purchaser of the program, authorized number of users, and time remaining for the annual software upgrade maintenance (SUM) and help agreement if purchased with ARROW. 


\subsection{Problem Reporting}

AFT ARROW provides an extensive context searchable online help manual and access to additional help, frequently asked questions (FAQ's), and modeling tips via the internet (www.aft.com).

Modeling problems, bug reports, and general pleas for assistance can be supplied to AFT via support (support@aft.com). A bug report is first verified and then acknowledged usually within two to three business days. The response may or may not solve the problem, but may provide a workaround until the problem is fixed. If requested, a special version of ARROW containing the bug fix (when available) can be supplied to the customer. If the error(s) persist, a special version of ARROW containing an extensive set of debugging tools can be supplied for use by the customers in an attempt to resolve the problem.

Users are notified via email when an update to ARROW is available. Minor updates are received several times a year. Major updates are received approximately once a year. Most of the minor revisions address bugs that have been found and corrected. The major once-a-year revisions usually deal with major enhancements and new capabilities that have been added. Whether or not to update depends on the time remaining in the annual SUM agreement, current workscope, and the nature of the bug fixes or enhancements.

\subsection{User Manual}

The user manual for AFT ARROW is a soft cover booklet running about 250 pages. The user manual is also available via online help. A single copy of the user manual was included with the software and is currently located at 2440 Stevens Center Room 1704. Additional copies can be purchased from Applied Flow Technology. 


\subsection{VALIDATION}

The ARROW user's manual contains a detailed discussion of the fundamental concepts and conventions used in processing piping systems and how to interpret the database information imbedded within ARROW (see Section 2.1). ARROW assumes that the user has a good general understanding of engineering hydraulics. ARROW is designed for use by persons who possess a level of knowledge consistent with that obtained from an undergraduate engineering course in the analysis of piping systems and fluid networks.

\subsection{Internal Databases}

Most engineering analyses involve processing very large amounts of information, some of which is critical, but most of which is of lessor importance. The key to analyze accurately and efficiently is the proper identification of the critical information. Two databases embedded within ARROW manage information associated with the fluid properties and pressure loss factors.

\subsubsection{Fluids}

Density and dynamic viscosity are two fluid properties required for all analyses. Fluid vapor pressure is optional. ARROW provides a fluid database for numerous common fluids. If the working fluid is not in the database, the user can enter fluid properties into a custom database for later recall.

Spot checks of the properties for air, helium, hydrogen, oxygen, nitrogen, and water against the values summarized in HOLMAN (1990) showed very good agreement.

\subsubsection{Losses}

ARROW provides hundreds of standard loss models based on well known references such as; Crane (1988), Idelchik (1994), and Miller (1990), but also allows the user to enter there own loss data. Variable or dynamic loss coefficients are supported. Variable loss coefficients may depend on flow, area ratios, and/or flow splits (such as tee's and wye's), and the angle of the connecting piping. ARROW also provides automatic calculation of friction factors using the well-known Colebrook-White correlation.

Spot checks of representative loss coefficients for valves and fittings against values summarized within Crane (1988) showed very good agreement.

\subsection{Test Problems}

Twenty-one sample problems (input files) are supplied with ARROW. All twenty-one input files are installed in the VERIFY sub-directory below the C: $\backslash A R R O W$ directory. The sample problems model systems that range in size from one pipe to seventy pipes. All of the sample problems are for systems with solutions available in published literature. Sample problem 5 requires the optional Chempak module for modeling mixtures and could not be run. 
Table 4-1 lists the sample problems and provides a brief description of each model. Appendix A summarizes the model, the reference, and the output test results. Appendix A contains copyrighted material. This material is printed with permission from Applied Flow Technology Corporation.

Table 4-1. Listing of Sample Problems Supplied with ARROW

\begin{tabular}{|c|c|c|}
\hline Number & Description & File Name \\
\hline 1 & $\begin{array}{l}1 \text { pipe, } 2 \text { junctions } \\
\text { Compressible air flow in a pipe. }\end{array}$ & Verify 1.aro \\
\hline 2 & $\begin{array}{l}1 \text { pipe, } 2 \text { junctions } \\
\text { Compressible methane flow in a pipe. }\end{array}$ & Verity2.aro \\
\hline 3 & $\begin{array}{l}1 \text { pipe, } 2 \text { junctions } \\
\text { Compressible steam flow in a pipe. }\end{array}$ & Verify3.aro \\
\hline 4 & $\begin{array}{l}1 \text { pipe, } 2 \text { junctions } \\
\text { Compressible air flow in a long pipe. }\end{array}$ & Verify4.aro \\
\hline 5 & $\begin{array}{l}1 \text { pipe, } 2 \text { junctions } \\
\text { Compressible natural gas (mixture) flow in a } 100- \\
\text { mile } 14 \text {-inch pipeline. Requires optional Chempak. } \\
\text { Problem. }\end{array}$ & Verify5.aro \\
\hline 6 & $\begin{array}{l}1 \text { pipe, } 2 \text { junctions } \\
\text { Compressible steam flow in a pipe. }\end{array}$ & Verify6.aro \\
\hline 7 & $\begin{array}{l}1 \text { pipe, } 2 \text { junctions } \\
\text { Compressible air flow in a pipe. }\end{array}$ & Verify 7.aro \\
\hline 8 & $\begin{array}{l}2 \text { pipes, } 3 \text { junctions } \\
\text { Compressible air flow in a branching network. }\end{array}$ & Verify8.aro \\
\hline 9 & $\begin{array}{l}1 \text { pipe, } 2 \text { junctions } \\
\text { Compressible air flow in a pipe. }\end{array}$ & Verify9.aro \\
\hline 10 & $\begin{array}{l}1 \text { pipe, } 2 \text { junctions } \\
\text { Compressible isothermal flow in a pipe. }\end{array}$ & Verify 10 aro \\
\hline 11 & $\begin{array}{l}1 \text { pipe, } 2 \text { junctions } \\
\text { Compressible air flow in a duct. }\end{array}$ & Verifyl1.aro \\
\hline 12 & $\begin{array}{l}1 \text { pipe, } 2 \text { junctions } \\
\text { Compressible air flow in a convergent nozzle. }\end{array}$ & Verify 12 .aro \\
\hline 13 & $\begin{array}{l}2 \text { pipes, } 4 \text { junctions } \\
\text { Compressible natural gas flow in a pipe. } \\
\text { Maximum length to prevent choked flow. }\end{array}$ & Verify 13 aro \\
\hline 14 & $\begin{array}{l}1 \text { pipe, } 2 \text { junctions } \\
\text { Compressible air flow in a pipe. Choked flow rate. }\end{array}$ & Verify 14 .aro \\
\hline 15 & $\begin{array}{l}1 \text { pipes, } 2 \text { junctions } \\
\text { Compressible air flow in a pipe. }\end{array}$ & Verify 15 .aro \\
\hline 16 & $\begin{array}{l}1 \text { pipes, } 2 \text { junctions } \\
\text { Compressible air flow in a pipe. Discharge pressure. }\end{array}$ & Verify 16.aro \\
\hline
\end{tabular}


SNF-5331, Rev. 0

\begin{tabular}{|c|l|c|}
\hline Number & \multicolumn{1}{|c|}{ Description } & File Name \\
\hline 17 & $\begin{array}{l}1 \text { pipes, 2 junctions } \\
\text { Compressible air flow in a pipe. Discharge pressure. }\end{array}$ & Verify 17.aro \\
\hline 18 & $\begin{array}{l}1 \text { pipes, 2 junctions } \\
\text { Compressible air flow in a pipe. Discharge pressure. }\end{array}$ & Verify18.aro \\
\hline 19 & $\begin{array}{l}1 \text { pipes, 2 junctions } \\
\text { Compressible air flow in a pipe. Discharge pressure. }\end{array}$ & Verify19.aro \\
\hline 20 & $\begin{array}{l}1 \text { pipes, 2 junctions } \\
\text { Compressible air flow in a pipe. Discharge pressure. }\end{array}$ & $\begin{array}{l}\text { Verify21.aro } \\
\text { Compressible air flow in a pipe. Discharge pressure. }\end{array}$ \\
\hline
\end{tabular}




\subsection{RESULTS}

Twenty-one sample problems were successfully executed and inspected. The input and output files are attached in Appendix A.

\subsection{Acceptance Criteria}

The following acceptance criteria were used to evaluate the results of the sample runs:

1) Each of the sample problems must execute and converge using the default or input supplied convergence criteria.

2) Each of the sample problems, with the exception of sample \#5, was compared to the published solutions.

\subsection{Test Results}

The pertinent results from each of the sample problems are summarized within the following tables and discussed within the following paragraphs. Most of the differences in flow rates and head losses were due to a combination of items: tighter tolerances (both global and individual pipes/junctions), friction factor models, and the rigorous treatment of pump heads (fitted polynomial versus linear interpolation). These differences were judged to reflect the improvements associated with the general state of knowledge now available for use within ARROW. These differences should not reflect adversely given the relatively simple hand calculations and techniques that were used to derive the published solutions.

\subsubsection{Sample Problem \#1}

Sample problem \# 1 consists of one pipe and two junctions. Sample problem \#1 models simple compressible adiabatic flow in a pipe from ANDERSON (1982). Air is the working gas.

The pipe is 30 meters in length with a hydraulic diameter of 0.15 meters. The friction factor is supplied as an explicit $\mathrm{f}(0.02)$. The problem states that the inlet mach number is 0.3 , the inlet pressure $P_{1}$ is 1 atm, and the inlet temperature $T_{1}$ is $273^{\circ} \mathrm{K}$. From the Ideal Gas Law: density, sonic speed, and mass flow rate can be calculated. Once the mass flow rate is known, it can be applied as an exit flow demand.

The results for sample problem \#1 are presented in Table 5-1. The corresponding results from ANDERSON (1982) are shown. Appendix A contains a detailed description of the sample problem and listings of both the input and output results. 
Table 5-1. Comparison of Results for Sample Problem \#1

\begin{tabular}{|l|c|c|}
\hline \multicolumn{1}{|c|}{ Parameter } & Anderson & AFT A rrow 2.0 \\
\hline$M_{2}$ - Mach number at exit & 0.475 & 0.4757 \\
\hline$P_{2}-$ Static pressure at exit (atm) & 0.624 & 0.6239 \\
\hline$T_{2}-$ Static temperature at exit $\left({ }^{\circ} \mathrm{K}\right)$ & 265.8 & 266.0 \\
\hline$P_{02}-$ Stagnation pressure at exit (atm) & 0.728 & 0.7279 \\
\hline
\end{tabular}

\subsubsection{Sample Problem \#2}

Sample problem \# 2 consists of one pipe and two junctions. Sample problem \#2 models compressible flow in a pipe from SAAD (1993). Methane is the working gas.

As specified, inlet conditions are known and the outlet conditions are sonic. The pipe length that yields sonic flow is the objective. With the known inlet conditions, an implied mass flow rate exists. To pose the problem in AFT Arrow terms; a few simple calculations are needed to obtain the mass flow rate. Once obtained, it is applied as a flow demand at the exit.

The problem states that the inlet velocity $V_{1}$ is $30 \mathrm{~m} / \mathrm{s}$, the inlet pressure $P_{1}$ is $0.8 \mathrm{MPa}$, and the inlet temperature $T_{1}$ is $320^{\circ} \mathrm{K}$. From the ideal gas law, density, sonic speed and mass flow rate can be determined. Once determine the mass flow rate can be applied as an exit demand. AFT ARROW does not solve for pipe length. Therefore, several manual iterations are required with different pipe lengths to obtain sonic flow $(M=1)$ at the exit.

The results for sample problem \#2 are presented in Table 5-2. The corresponding results from SAAD (1993) are shown. Appendix A contains a detailed description of the sample problem and listings of both the input and output results.

Table 5-2. Comparison of Results for Sample Problem \#2

\begin{tabular}{|l|c|c|}
\hline \multicolumn{1}{|c|}{ Parameter } & Saad & ArI A r row 2.0 \\
\hline Maximum pipe length $(\mathrm{m})$ & 4024 & 4029.49 \\
\hline$P_{2}-$ Static pressure at exit $(\mathrm{kPa})$ & 48.2 & 48.10 \\
\hline$V_{2}-$ Velocity at exit $(\mathrm{m} / \mathrm{s})$ & 433.17 & 433.13 \\
\hline$T_{2}-$ Static temperature at exit $\left({ }^{\circ} \mathrm{K}\right)$ & 278.43 & 277.88 \\
\hline
\end{tabular}




\subsubsection{Sample Problems \#3}

Sample problem \#3 consists of one pipe and two junctions. Sample problem \#3 models 600-psig steam at $850^{\circ} \mathrm{F}$ flowing through a system with 400 feet of horizontal 6-inch Schedule 80 pipe at a rate of 90,000 pounds per hour.

The results for sample problem \#3 are presented in Table 5-3. Comparison is made to the published results from CRANE (1988). Appendix A contains a detailed description of the sample problem and listings of both the input and output results.

Table 5-3. Comparison of Results for Sample Problem \#3

\begin{tabular}{|l|c|c|}
\hline Parameter & Crane & AFI Arrow 2,0 \\
\hline Static pressure drop (psi) & 40.1 & 41.59 \\
\hline
\end{tabular}

\subsubsection{Sample Problem \#4}

Sample problem \#4 consists of one pipe and two junctions. Sample Problem \#4 models air flowing at 65 -psig steam and $110^{\circ} \mathrm{F}$ through 75 feet of 1 -inch Schedule 40 pipe at a rate of 100 standard cubic feet per minute (scfm).

The results for sample problem \#4 are presented in Table 5-4. Comparison is made to the published results from CRANE (1988). Appendix A contains a detailed description of the sample problem and listings of both the input and output results.

Table 5-4. Comparison of Results for Sample Problem \#4

\begin{tabular}{|c|c|c|}
\hline Parameter & Crane & APT A mov 20 \\
\hline Static pressure drop (psi) & 2.61 & 2.712 \\
\hline Volumetric flow rate at inlet (ft3/min) & 20.2 & 20.21 \\
\hline Volumetric flow rate at outlet (ft3/min) & 20.9 & 20.93 \\
\hline Velocity inlet (ft/min) & 3,367 & 3,368 \\
\hline Velocity inlet (ft/min) & 3,483 & 3,487 \\
\hline
\end{tabular}

\subsubsection{Sample Problem \#5}

Sample Problem 5 could not be run because it requires the optional Chempak module. Table 5-5 was purposely left blank. 
The results for sample problem \#5, as supplied by Applied Flow Technology, are presented in Appendix A. Comparison is made to the published results from CRANE (1988).

\section{Table 5-5. Comparison of Results for Sample Problem \#5}

\subsubsection{Sample Problem \#6}

Sample problem \#6 consists of one pipe and two junctions. Sample Problem \#6 models saturated steam at 170-psia feeding a pulp digester through and 30 feet of 1-inch Schedule 40.

The results for sample problem \#6 are presented in Table 5-6. Comparison is made to the published results from CRANE (1988). Appendix A contains a detailed description of the sample problem and listings of both the input and output results.

Table 5-6. Comparison of Results for Sample Problem \#6

\begin{tabular}{|c|c|c|c|}
\hline Parameter & $\begin{array}{l}\text { Crane Modined } \\
\text { Darcy Morinula }\end{array}$ & $\begin{array}{l}\text { Crame Sonic } \\
\text { Velocity } \text { Pormula }\end{array}$ & KOHT \\
\hline Mass flow rate (lbm/hr) & 11,780 & 11,180 & 11,042 \\
\hline Exit static enthalpy (Btu/lbm) & $\mathrm{N} / \mathrm{A}$ & 1,196 & $1,147.6$ \\
\hline Exit static temperature $\left({ }^{\circ} \mathrm{F}\right)$ & N/A & 317 & 240.7 \\
\hline
\end{tabular}

\subsubsection{Sample Problem \#7}

Sample problem \#7 consists of one pipe and two junctions. Air supplied at a pressure of 19.3pisg and a temperature of $100^{\circ} \mathrm{F}$ is measured at a point 10 feet from the outlet of $1 / 2$-inch Schedule 80 pipe discharging to the atmosphere.

The results for sample problem \#7 are presented in Table 5-7. Comparison is made to the published results from CRANE (1988). The Crane prediction indicates that this pipe will have subsonic velocity at the exit and hence will not choke. However, a more proper formulation of this problem shows that sonic choking will occur. This is a good example of the limitations of simplified methods such as Crane. The discrepancy comes from how to handle the exit loss of the air as it discharges to atmosphere. The Crane solution takes the appropriate $\mathrm{K}$ factor, equal to 1 , and lumps it together with the pipe friction to obtain an overall $\mathrm{K}$ factor of 7.04 . However, if the $\mathrm{K}$ factor is applied at the discharge tank and not averaged along the pipe, the flow chokes. The predicted choked flow rate is $64.3 \mathrm{scfm}$. This is not drastically different from Crane's prediction, and well within typical engineering uncertainty. However, the difference, which is small here, could be larger in other applications.

Appendix A contains a detailed description of the sample problem and listings of both the input and output results. 
Table 5-7. Comparison of Results for Sample Problem \#7

\begin{tabular}{|c|c|c|}
\hline Parameter & Crane & AFT Arrow 2.0 \\
\hline Mass flow rate (scfm) & 62.7 & 63.3 \\
\hline
\end{tabular}

\subsubsection{Sample Problem \#8}

Sample problem \#8 consists of two pipes and three junctions. Sample problem \#8 represents a branching system where the two pipe lengths necessary to obtain a measured pressure and choked flow are unknown. Air is the working gas.

ARROW does not solve for pipe length. However, the length of Pipe \#1 can be manually changed to obtain the measured pressure. The resulting mass flow rate is then used as input for Pipe \#2.

The results are presented in Table 5-8. Comparison is made to the published results from FOX (1985). The results agree very closely. Appendix A contains a detailed description of the sample problem and listings of both the input and output results.

Table 5-8. Comparison of Results of Sample Problem \#8

\begin{tabular}{|l|c|c|}
\hline \multicolumn{1}{|c|}{ Parameter } & Fox $\alpha$ McDonald & AFT Arrow 2.0 \\
\hline$L_{1-3}-$ length to choking $(\mathrm{m})$ & 4.99 & 4.994 \\
\hline$V_{1}-$ Velocity at point $1(\mathrm{~m} / \mathrm{s})$ & 65.3 & 65.2 \\
\hline$T_{1}-$ Temperature at point $1\left({ }^{\circ} \mathrm{K}\right)$ & 294 & 293.9 \\
\hline$M_{1}-$ Mach number at point 1 & 0.19 & 0.19 \\
\hline$M_{2}$ - Mach number at point 2 & 0.40 & 0.40 \\
\hline$P_{2}-$ Pressure at point $2(\mathrm{~mm} \mathrm{Hg}$ gauge) & -18.9 & $-18.86^{*}$ \\
\hline$L_{1-2}$ - length to measured pressure $(\mathrm{m})$ & 4.29 & $4.29^{*}$ \\
\hline
\end{tabular}

* AFT Arrow does not have the ability to solve for pipe length, so the length was manually input. With this known length, the AFT Arrow Mach number at $\mathbf{M}_{2}$ should agree with Fox \& McDonald's, and it does. The resulting mass flow rate is then used as input for AFT Arrow pipe \#2. 


\subsubsection{Sample Problem \#9}

Sample problem \#9 consists of one pipe and two junctions. As specified, inlet conditions are known and outlet conditions need to be determined. With the known inlet conditions, an implied mass flow rate exists. To pose the problem in AFT Arrow terms a few simple calculations are needed to obtain the mass flow rate. Once obtained, it is applied as a flow demand at the exit.

The results are presented in Table 5-9. Comparison is made to the published results from LINDEBURG (1984). Appendix A contains a detailed description of the sample problem and listings of both the input and output results.

Table 5-9. Comparison of Results of Sample Problem \#9

\begin{tabular}{|c|c|c|}
\hline Parameter & Lindeburg & AFT Arrow 2.0 \\
\hline$M_{2}$ - Mach number at exit & 0.35 & 0.356 \\
\hline$P_{2}-$ Pressure at exit (psia) & 10.26 & 10.09 \\
\hline
\end{tabular}

\subsubsection{Sample Problem \#10}

Sample problem \#10 consists of one pipe and two junctions. Methane is the working gas. The problem states that the pipe length is 75 miles and 40 inches in diameter with an explicit friction factor of 0.00936 . The inlet pressure $P_{1}$ is 650 -psia. The outlet pressure $P_{2}$ is 450-psia. Determine the mass flow rate.

The results are presented in Table 5-10. Comparison is made to the published results from LINDEBURG (1984). Appendix A contains a detailed description of the sample problem and listings of both the input and output results.

Table 5-10. Comparison of Results for Sample Problem \#10

\begin{tabular}{|c|c|c|}
\hline Parameter & Lindeburg & AFT A rrow 2.0. \\
\hline Mass flow rate $(\mathrm{lbm} / \mathrm{s})$ & 456 & 478 \\
\hline
\end{tabular}

\subsubsection{Sample Problem \#11}

Sample problem \#11 consists of one pipe and two junctions. Air is the working gas.

Exit conditions are known. The inlet conditions need to be determined for the specified volumetric flow. With the known exit conditions, a few simple calculations are needed to obtain the implied mass flow rate. Once obtained, it is applied as an inlet flow demand.

In AFT Arrow, the discharge pressure can be specified. The temperature can be specified at the exit junction, but the actual discharge is what is displayed for the pipe exit. The pipe exit 
temperature depends on the inlet temperature and the thermodynamic process in the pipe, which is adiabatic. Therefore, to solve this problem the inlet static temperature at $\mathrm{J} 1$ must be guessed until the pipe delivers $293 \mathrm{~K}$ at the exit.

The results are presented in Table 5-11. Comparison is made to the published results from SAAD (1993). Appendix A contains a detailed description of the sample problem and listings of both the input and output results.

Table 5-11. Comparison of Results for Sample Problem \#11

\begin{tabular}{|l|c|c|}
\hline Parameter & Saad & A F T A row 2.0 \\
\hline$M_{2}-$ Mach number at exit & 0.685 & 0.689 \\
\hline$M_{1}-$ Mach number at inlet & 0.347 & 0.347 \\
\hline$P_{1}-$ Static pressure at inlet $(\mathrm{kPa})$ & 306 & 308.1 \\
\hline$T_{1}-$ Static temperature at inlet $\left({ }^{\circ} \mathrm{K}\right)$ & 312.76 & 312.9 \\
\hline
\end{tabular}

\subsubsection{Sample Problem \#12}

Sample problem \#12 consists of one pipe and two junctions. Air is the working gas. Inlet conditions are known. Air flows isentropically through a convergent nozzle which feeds an insulated constant are duct.

The results are presented in Table 5-12. Comparison is made to the published results from SAAD (1993). Appendix A contains a detailed description of the sample problem and listings of both the input and output results.

Table 5-12. Comparison of Results for Sample Problem \#12

\begin{tabular}{|c|c|c|}
\hline Parameter & Saad & A AP I Irow 20 \\
\hline Mass flow rate when choked $(\mathrm{kg} / \mathrm{s})$ & 2.11 & 2.10 \\
\hline$M_{1}-$ Mach number at inlet & 0.603 & 0.604 \\
\hline$P_{1}-$ Static pressure at inlet $(\mathrm{MPa})$ & 2.106 & 2.114 \\
\hline$T_{1}-$ Static temperature at inlet $\left({ }^{\circ} \mathrm{K}\right)$ & 427.8 & 429.6 \\
\hline$P_{2, \text { choke }}-$ Static back pressure for choking (MPa) & 1.203 & 1.217 \\
\hline
\end{tabular}




\subsubsection{Sample Problem \#13}

Sample problem \#13 consists of two pipes and four junctions. Natural gas is the working gas. Inlet conditions are known. Outlet conditions are to be determined. With the known inlet conditions, a few simple calculations are needed to obtain the implied mass flow rate. Once obtained, it is applied as an outlet flow demand.

The first pipe represents the pipe from point 1 to 2 . The second pipe represents the pipe from point 1 to the choking pint. AFT Arrow does not explicitly solve for pipe length. To obtain the maximum pipe length, different lengths must be guessed with lengths that exceed sonic flow discarded.

The results are presented in Table 5-13. Comparison is made to the published results from SAAD (1993). All results agree closely. The AFT Arrow static pressure below which choking occurs is the pipe exit static pressure. Appendix A contains a detailed description of the sample problem and listings of both the input and output results.

Table 5-13. Comparison of Results for Sample Problem \#13

\begin{tabular}{|l|c|c|}
\hline \multicolumn{1}{|c|}{ Parameter } & Saad & AFT Arrow 2.0 \\
\hline$P_{2}-$ Static pressure at exit $(\mathrm{kPa})$ & 263.16 & 262.0 \\
\hline$M_{2}-$ Mach number at exit & 0.38 & 0.3818 \\
\hline$L_{1-2}-$ Length to reach $500 \mathrm{kPa}(\mathrm{m})$ & 544.79 & 558.5 \\
\hline$L_{\mathrm{T}}{ }^{*}-$ Length to reach sonic choke point $(\mathrm{m})$ & 710 & 709.8 \\
\hline$P_{\mathrm{T}}{ }^{*}-$ Static pressure choke point $(\mathrm{kPa})$ & 114.46 & 114.9 \\
\hline$M_{\mathrm{T}}{ }^{*}-$ Mach number at isothermal choke point & 0.874 & 0.8704 \\
\hline
\end{tabular}

\subsubsection{Sample Problem \#14}

Sample problem \#14 consists of one pipe and two junctions. The objective is to solve for the choked flow rate. Air is the working gas.

The results are presented in Table 5-14. Comparison is made to the published results from PERRY. Appendix A contains a detailed description of the sample problem and listings of both the input and output results.

Table 5-14. Comparison of Results for Sample Problem \#14

\begin{tabular}{|c|c|c|}
\hline Parameter & Perry & AFT Krow 20 \\
\hline Mass flow rate $(\mathrm{kg} / \mathrm{s})$ & 2.7 & 2.716 \\
\hline
\end{tabular}




\subsubsection{Sample Problem \#15}

Sample problem \#15 consists of one pipe and two junctions. Air is the working gas. Inlet conditions are known. Outlet conditions need to be determined. With the known inlet conditions, a few simple calculations are needed to obtain the implied mass flow rate. Once obtained, it is applied as an outlet flow demand.

The results are presented in Table 5-15. Comparison is made to the published results from JANNA (1983). Appendix A contains a detailed description of the sample problem and listings of both the input and output results.

Table 5-15. Comparison of Results for Sample problem \#15

\begin{tabular}{|c|c|c|}
\hline Parameter & $14 \min$ & 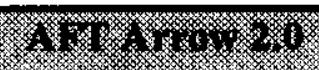 \\
\hline$M_{2}-$ Mach number at exit & 0.14 & 0.1355 \\
\hline$P_{2}-$ Pressure at exit (psia) & 9.63 & 9.836 \\
\hline$T_{2}-$ Temperature at exit $\left({ }^{\circ} \mathrm{R}\right)$ & 528.8 & 528.9 \\
\hline
\end{tabular}

\subsubsection{Sample Problem \#16}

Sample problem \#16 consists of one pipe and two junctions. Air is discharged from a highpressure tank through a 4-inch (Schedule 40) vent line into the atmosphere. The mass flow is given as $72,000 \mathrm{lbm} / \mathrm{hr}$. Stagnation conditions within the tank are $P_{0}=600$-psig (constant) and $\mathrm{T}_{\mathrm{o}}=120^{\circ} \mathrm{F}$. The equivalent length of the pipe is 90 feet.

The results are presented in Table 5-16. Comparison is made to the published results from NAYYAR (1992). Appendix A contains a detailed description of the sample problem and listings of both the input and output results.

Table 5-16. Comparison of Results for Sample Problem \#16

\begin{tabular}{|c|c|c|}
\hline Parameter & (1) & . \\
\hline$M_{1}-$ Mach number at valve & 0.317 & 0.318 \\
\hline$P_{1}-$ Pressure at valve (psia) & 128.46 & 128.66 \\
\hline
\end{tabular}

\subsubsection{Sample Problem \#17}

Sample problem \#17 is identical to sample problem \#16, except that the mass flow rate is doubled. 
The results are presented in Table 5-17. Comparison is made to the published results from SAAD (1992). Appendix A contains a detailed description of the sample problem and listings of both the input and output results.

Table 5-17. Comparison of Results for Sample Problem \#17

\begin{tabular}{|l|c|c|}
\hline$M_{1}-$ Mach number at valve & Nay & A PT Arrow 20 \\
\hline$P_{1}-$ Pressure at valve (psia) & 0.317 & 0.318 \\
\hline
\end{tabular}

\subsubsection{Sample Problem \#18}

Sample problem \#18 is identical to sample problem \#16, except that the pipe length is doubled.

The results are presented in Table 5-18. Comparison is made to the published results from SAAD (1992). Appendix A contains a detailed description of the sample problem and listings of both the input and output results.

Table 5-18. Comparison of Results for Sample Problem \#18

\begin{tabular}{|l|c|c|}
\hline$M_{1}-$ Mach number at valve & Nayyar & AFI A r row 20 \\
\hline$P_{1}-$ Pressure at valve (psia) & 0.243 & 0.243 \\
\hline
\end{tabular}

\subsubsection{Sample Problem \#19}

Sample problem \#19 is identical to sample problem \#16, except that the air temperature is 500 ${ }^{\circ} \mathrm{F}$.

The results are presented in Table 5-19. Comparison is made to the published results from SAAD (1992). Appendix A contains a detailed description of the sample problem and listings of both the input and output results.

Table 5-19. Comparison of results for Sample Problem \#19

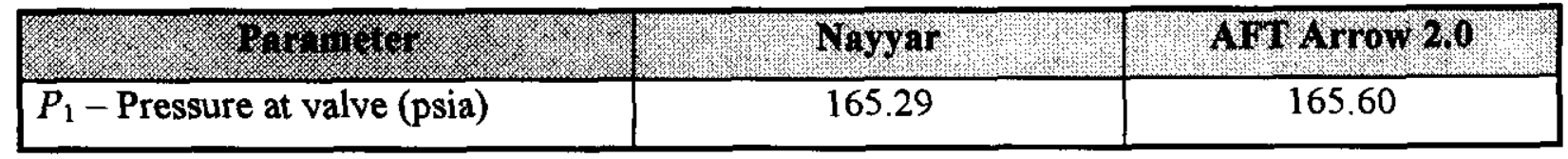




\subsubsection{Sample Problem \#20}

Sample problem $\# 20$ is identical to sample problem $\# 16$, except that the pipe diameter has been decreased to 2 -inches.

The results are presented in Table 5-20. Comparison is made to the published results from SAAD (1992). Appendix A contains a detailed description of the sample problem and listings of both the input and output results.

Table 5-20. Comparison of Results for Sample problem \#20

\begin{tabular}{|l|c|c|}
\hline \multicolumn{1}{|c|}{ Parameter } & Naygar & APT A row 2.0 \\
\hline$M_{1}$ - Mach number at valve & 0.235 & 0.235 \\
\hline$P_{1}-$ Pressure at valve (psia) & 661.96 & 662.56 \\
\hline
\end{tabular}

\subsubsection{Sample Problem \#21}

Sample problem \#21 is identical to sample problem \#16, except that the pipe diameter has been increased to 8-inches.

The results are presented in Table 5-21. Comparison is made to the published results from SAAD (1992). Appendix A contains a detailed description of the sample problem and listings of both the input and output results.

Table 5-21. Comparison of Results for Sample Problem \#21

\begin{tabular}{|l|c|c|}
\hline \multicolumn{1}{|c|}{ Parameter } & Nayyar & AFT A rrow 2.0 \\
\hline$M_{1}$ - Mach number at valve & 0.4096 & 0.4142 \\
\hline$P_{1}-$ Pressure at valve (psia) & 25.15 & 24.95 \\
\hline
\end{tabular}




\subsection{SUMMARY AND CONCLUSIONS}

The ARROW (Version 2) compressible flow network analysis program has been installed and successfully validated for use. Based on a direct comparison of twenty of twenty-one sample problems to published results, the ARROW computer program is judged acceptable for use at the Hanford site. Furthermore, the use of an encrypted license key to assures proper installation and configuration control

The pertinent results from each of the sample problems have been summarized within the preceding tables. It must be noted that in most cases very good agreement was found. However, a few cases of significant differences between ARROW and the published results were found. Most of the differences in flow rates and pressures were due to a combination of items: tighter tolerances (both global and individual pipes/junctions), friction factor models, and the rigorous treatment component losses

These differences were judged to reflect the improvements associated with the general state of knowledge now automated and available for use within ARROW. These differences should not reflect adversely given the relatively simple hand calculations and techniques that were used to derive the published solutions. 


\subsection{REFERENCES}

Anderson, J. D., "Modern Compressible Flow”, McGraw-Hill, 1982.

"Flow of Fluids Through Valves, Fittings, and Pipe", Technical Paper Number 410, Crane Company, Chicago, Illinois, 1991.

Fox, R. W. and A. T. McDonald, "Introduction to Fluid Mechanics", Third Edition, 1985, p. 376.

Holman, J. P., "Heat Transfer", Seventh Edition, McGraw-Hill, Inc., New York, New York, 1990.

Idelchik, I. E., "Handbook of Hydraulic Resistance", Third Edition, CRC Press, Ann Arbor, Michigan, 1994.

Janna, W. S., "Introduction to Fluid Mechanics", PWS Publishers, Belmont, CA.

Lindeburg, M. R., "Mechanical Engineering Review Manual for the P. E. Exam", 7th Ed., 1984.

Miller, D. S., "Internal Flow Systems", Second Edition, Published by BHRA (Information Services), Cranfield, UK, 1990.

Marks Standard Handbook for Mechanical Engineers, 8th ed., 1978, T. Baumeister, editor.

Nayyar, M. L., "Piping Handbook", Sixth Edition, McGraw-Hill, New York, 1992.

Perry, R. H. and D. W. Green, "Perry's Chemical Engineer's Handbook", Seventh Edition.

Saad, M. A., "Compressible Fluid Flow", Second Edition, Prentice Hall, Englewood Cliffs, New Jersey, 1993. 
SNF-5331, Rev. 0

\section{APPENDIX A - SAMPLE PROBLEM DESCRIPTIONS, INPUT, AND OUTPUT}

Appendix A contains copyright material. Problem descriptions were printed with permission from Applied Flow Technology Corporation. 
TITLE: VERIFY1.ARO

REFERENCE: John D. Anderson, Modern Compressible Flow, 1982, McGraw-Hill, page 76, example . 3.4

GAS: Air

ASSUMPTIONS: 1) Adiabatic, 2) Perfect gas

RESULTS:

\begin{tabular}{|l|r|r|}
\hline \multicolumn{1}{|c|}{ Parameter } & Anderson & AFT Arrow 2.0 \\
\hline$M_{2}$ - Mach number at exit & 0.475 & 0.4757 \\
\hline$P_{2}-$ Static pressure at exit (atm) & 0.624 & 0.6239 \\
\hline$T_{2}$ - Static temperature at exit (deg. K) & 265.8 & 266.0 \\
\hline$P_{\mathrm{o} 2}-$ Stagnation pressure at exit (atm) & 0.728 & 0.7279 \\
\hline
\end{tabular}

\section{DISCUSSION:}

As specified, inlet conditions are known and outlet conditions need to be determined. With the known inlet conditions, an implied mass flow rate exists. To pose the problem in AFT Arrow terms, a few simple calculations are needed to obtain the mass flow rate. Once obtained, it is applied as a flow demand at the exit.

The problem states that the inlet Mach number is $0.3, P_{1}=1 \mathrm{~atm}, T_{1}=273 \mathrm{~K}$. From the ideal gas law, density, sonic speed and mass flow rate are:

$$
\begin{aligned}
& \rho_{1}=\frac{P_{1}}{R T_{1}}=\frac{1 \mathrm{~atm}}{\left(.2868 \frac{\mathrm{kJ}}{\mathrm{kg} \cdot \mathrm{K}}\right)(273 \mathrm{~K})}=1.293 \mathrm{~kg} / \mathrm{m}^{3} \\
& a_{1}=\sqrt{\gamma R T_{1}}=\sqrt{1.4\left(287 \frac{\mathrm{J}}{\mathrm{kg} \cdot \mathrm{K}}\right) 273 \mathrm{~K}}=331.2 \mathrm{~m} / \mathrm{s} \text { (sonic velocity) } \\
& \dot{m}=\rho_{1} V_{1} A=\rho_{1}\left(M_{1} a_{1}\right) A=\left(1.293 \frac{\mathrm{kg}}{\mathrm{m}^{3}}\right)(0.3)\left(331.2 \frac{\mathrm{m}}{\mathrm{s}}\right)\left(\frac{\pi}{4} 0.15^{2} \mathrm{~m}^{2}\right)=2.27 \mathrm{~kg} / \mathrm{s}
\end{aligned}
$$

Note that the friction factor in Anderson is the Fanning friction factor. To obtain the Darcy-Weisbach friction factor used in AFT Arrow, multiply the Fanning friction factor by 4 .

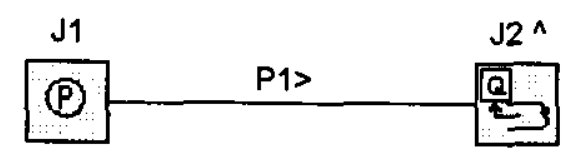

Copyright 01999 Applied Flow Technology Corp. All rights reserved. 
John D. Anderson

Modern Compressible Flow, 1982, McGraw-Hill

page 76 , example 3.4

Air flow in a pipe - Example assumes adiabatic flow and air is a perfect gas

See Verify1.doc MS Word file for comparison with published results

Title: Verify1.aro - Modern Compressible Flow, Example 3.4, pg 76

Number Of Pipes $=1$

Number Of Junctions $=2$

Length March Solution Method with Mach Number Limits

Segments Per Pipe= 2

Mach Number Increment= .01

Pressure Tolerance $=.0001$ relative change

Mass Flow Rate Tolerance $=.0001$ relative change

Enthalpy Tolerance $=.0001$ relative change

Concentration Tolerance $=.0001$ relative change

Flow Relaxation= (Automatic)

Pressure Relaxation= (Automatic)

Fluid Database: AFT Standard

Fluid $=$ Air

Max Fluid Temperature Data $=1000$ deg. $K$

Min Fluid Temperature Data $=200$ deg. $\mathrm{K}$

Molecular Weight $=28.97 \mathrm{amu}$

Gas Constant $=0.2868 \mathrm{~kJ} / \mathrm{kg}-\mathrm{K}$

Critical Pressure $=37.25 \mathrm{~atm}$

Critical Temperature $=132.41 \mathrm{deg} . \mathrm{K}$

Acentric Factor $=.021$

Equation of State $=\mid$ deal Gas

Enthalpy Model $=$ Reference

Specific Heat Ratio Accuracy= High

Atmospheric Pressure $=1 \mathrm{~atm}$

Gravitational Acceleration $=1 \mathrm{~g}$ 's

Standard Pressure $=14.696$ psia

Standard Temperature $=60 \mathrm{deg} . F$

Turbulent Flow Above Reynolds Number $=4000$

Laminar Flow Below Reynolds Number $=2300$

Specific Heat Ratio Accuracy $=$ High

Atmospheric Pressure $=1$ atm

Gravitational Acceleration $=1 \mathrm{~g}$

Standard Pressure $=14.696$ psia

Standard Temperature $=60 \mathrm{deg} . \mathrm{F}$

Turbulent Flow Above Reynolds Number $=4000$

Laminar Flow Below Reynolds Number $=2300$

\section{Pipe Input Table}

\begin{tabular}{|c|c|r|r|r|r|r|r|r|r|r|}
\hline Pipe & Name & $\begin{array}{c}\text { Pipe } \\
\text { Defined }\end{array}$ & Length & $\begin{array}{c}\text { Length } \\
\text { Units }\end{array}$ & $\begin{array}{c}\text { Hydraulic } \\
\text { Diameter }\end{array}$ & $\begin{array}{c}\text { Hydraulic } \\
\text { Diam. Units }\end{array}$ & Roughness & $\begin{array}{c}\text { Roughness } \\
\text { Units }\end{array}$ & Losses (K) & Initial Flow \\
\hline 1 & Pipe & Yes & 30 & meters & 0.15 & meters & 0.02 & Explicit $f$ & 0 & \\
\hline
\end{tabular}

\begin{tabular}{|c|c|c|c|c|c|c|c|}
\hline Pipe & $\begin{array}{c}\text { Initial Flow } \\
\text { Units }\end{array}$ & $\begin{array}{c}\text { Junctions } \\
\text { (Up,Down) }\end{array}$ & Geometry & Material & Size & Type & $\begin{array}{c}\text { Special } \\
\text { Condition }\end{array}$ \\
\hline 1 & & 1,2 & Cylindrical Pipe & Unspecified & & & None \\
\hline
\end{tabular}

\section{Assigned Flow Table}


SNF-5331, Rov. 0

AFT Arrow 2.0 Input

Numatec Hanford Corporation
(2)

$12 \pi / 99$ 10:10 AM

Verify1.aro - Modern Compressible Flow, Example 3.4, pg 76

\begin{tabular}{|c|c|c|c|c|c|c|c|c|c|c|}
\hline Assigned Flow & Name & $\begin{array}{l}\text { Object } \\
\text { Defined }\end{array}$ & $\begin{array}{c}\text { Database } \\
\text { Source }\end{array}$ & $\begin{array}{c}\text { Special } \\
\text { Condition } \\
\end{array}$ & Fluid & Type & Flow & $\begin{array}{l}\text { Flow } \\
\text { Units }\end{array}$ & Temperature & $\begin{array}{c}\text { Temperature } \\
\text { Units }\end{array}$ \\
\hline 2 & Assigned Flow & Yes & & None & Air & Outflow & 2.27 & $\mathrm{~kg} / \mathrm{sec}$ & 273 & $\operatorname{deg} . \mathrm{K}$ \\
\hline
\end{tabular}

\begin{tabular}{|c|r|}
\hline Assigned Flow & $\begin{array}{c}\text { Loss } \\
\text { Model }\end{array}$ \\
\hline 2 & 0 \\
\hline
\end{tabular}

Assigned Pressure Table

\begin{tabular}{|c|c|r|r|r|r|r|r|r|}
\hline Assigned Pressure & Name & $\begin{array}{c}\text { Object } \\
\text { Defined }\end{array}$ & $\begin{array}{c}\text { Database } \\
\text { Source }\end{array}$ & $\begin{array}{c}\text { Special } \\
\text { Condition }\end{array}$ & Fluid & Pressure & $\begin{array}{c}\text { Pressure } \\
\text { Units }\end{array}$ & \begin{tabular}{c} 
Temperature \\
\hline 1
\end{tabular} \\
\hline 1 & Assigned Pressure & Yes & & N/A & Air & 1 & atm & 273 \\
\hline
\end{tabular}

\begin{tabular}{|c|r|r|}
\hline Assigned Pressure & $\begin{array}{c}\text { Temperature } \\
\text { Units }\end{array}$ & $\begin{array}{c}\text { Loss } \\
\text { Factor }\end{array}$ \\
\hline 1 & deg. K & 0 \\
\hline
\end{tabular}


John D. Anderson

Modern Compressible Flow, 1982, McGraw-Hill

page 76, example 3.4

Air flow in a pipe - Example assumes adiabatic flow and air is a perfect gas

See Verify1.doc MS Word file for comparison with published reaults

Title: Verify1.aro - Modern Compressible Flow, Example 3.4, pg 76

Analysis run on: 12/799 10:11:29 AM

Input File: C:VFT ProductsLAFT ArrowlVerificationlverify1,aro

Execution Timex 0.27 seconds

Total Number Of Pressure Iterations= 0

Total Number Of Flow Iterations= 2

Total Number Of Enthalpy Iterations= 2

Number Of Pipes= 1

Number Of Junctions $=2$

Length March Solution Method with Mach Number Limits

Segments Per Pipe= 2

Mach Number Increment $=.01$

Pressure Tolerance= .0001 relative change

Mass Flow Rate Tolerance $=.0001$ relative change

Enthalpy Tolerance $=.0001$ relative change

Flow Relaxation= (Automatic)

Pressure Relaxation= (Automatic)

Fluid Database: AFT Standard

Fluid= Air

Max Fluid Temperature Data $=1000$ deg. $K$

Min Fluid Temperature Data $=200$ deg. $\mathrm{K}$

Molecular Weight $=28.97$ amu

Gas Constant $=0.2868 \mathrm{~kJ} / \mathrm{kg}-\mathrm{K}$

Critical Pressure $=37.25 \mathrm{~atm}$

Critical Temperature= $132.41 \mathrm{deg} . \mathrm{K}$

Acentric Factor $=.021$

Equation of State= Ideal Gas

Enthalpy Model= Reference

Specific Heat Ratio Accuracy= High

Atmospheric Pressure= $1 \mathrm{~atm}$

Gravitational Acceleration $=1 \mathrm{~g} / \mathrm{s}$

Standard Pressure= 14.696 psia

Standard Temperature $=60$ deg. $F$

Turbulent Flow Above Reynolds Number $=\mathbf{4 0 0 0}$

Laminar Flow Below Reynolds Number $=\mathbf{2 3 0 0}$

Specific Heat Ratio Accuracy= High

Atmospheric Pressure $=1 \mathrm{~atm}$

Gravitational Acceleration $=1 \mathrm{~g}$

Standard Pressure $=14.696$ psia

Standard Temperature $=60 \mathrm{deg} . F$

Turbulent Flow Above Reynolds Number $=4000$

Laminar Flow Below Reynolds Number $=2300$

Overall Delta Pressure $=-0.03409 \mathrm{MPa}$

Total Inflow $=2.270 \mathrm{~kg} / \mathrm{sec}$

Total Outflow $=2.270 \mathrm{~kg} / \mathrm{sec}$

Total Energy Inflow $=992.7 \mathrm{~kW}$

Total Energy Outflow $=992.7 \mathrm{~kW}$

Total Heat Transferred Into Systern $=0.000 \mathrm{~kW}$

Maximum Pressure is $0.1013 \mathrm{MPa}$ at Junction 1 Inlet

Minimum Pressure is $0.06322 \mathrm{MPa}$ at Junction 2 Inlet

Maximum Static Temperature is $4.703 \mathrm{deg}$. $C$ at Junction 2 Inlet

Minimum Static Temperature is $4.699 \mathrm{deg}$. C at Junction 1 Inlet

\section{Pipe Output Table}


SNF-5331, Rev. 0

AFT Arrow 2.0 Output

(2)

Verify1.aro - Modern Compressible Flow, Example 3.4, pg 76

\begin{tabular}{|c|c|c|c|c|c|c|c|c|c|c|}
\hline Pipe & $\begin{array}{c}\text { Mach } \\
\text { \# Out }\end{array}$ & $\begin{array}{c}\text { P Static } \\
\text { Out } \\
\text { (atm) }\end{array}$ & $\begin{array}{c}\text { P Stag. } \\
\text { Out } \\
(\mathrm{atm})\end{array}$ & $\begin{array}{c}\text { T Stag. } \\
\text { Out } \\
\text { (deg. K) }\end{array}$ & $\begin{array}{c}\text { T Static } \\
\text { Out } \\
\text { (deg. K) }\end{array}$ & $\begin{array}{c}\text { P Stag. } \\
\text { In } \\
(\mathrm{atm})\end{array}$ & $\begin{array}{c}\text { Mass } \\
\text { Flow } \\
(\mathrm{kg} / \mathrm{sec})\end{array}$ & $\begin{array}{c}\text { Rho } \\
\text { Static In } \\
(\mathrm{kg} / \mathrm{m} 3)\end{array}$ & $\begin{array}{c}\text { Vel. } \\
\text { In } \\
(\mathrm{meters} / \mathrm{sec})\end{array}$ & $\begin{array}{c}\text { Vel. } \\
\text { Sonic In } \\
(\mathrm{meters} / \mathrm{sec})\end{array}$ \\
\hline 1 & 0.4757 & 0.6239 & 0.7279 & 277.9 & 266.0 & 1.064 & 2.270 & 1.293 & 99.33 & 330.4 \\
\hline
\end{tabular}

All Junction Table

\begin{tabular}{|c|c|c|r|r|}
\hline Jct & $\begin{array}{c}\text { P Stag. } \\
\text { In } \\
\text { (atm) }\end{array}$ & $\begin{array}{c}\text { P Static } \\
\text { In } \\
\text { (atm) }\end{array}$ & $\begin{array}{c}\text { T Static } \\
\text { In } \\
\text { (deg. K) }\end{array}$ & $\begin{array}{c}\text { T Stag. } \\
\text { In } \\
\text { (deg. K) }\end{array}$ \\
\hline 1 & 1.0644 & 1.0000 & 273.0 & 277.8 \\
\hline 2 & 0.7279 & 0.6239 & 266.0 & 277.9 \\
\hline
\end{tabular}




\section{TITLE: VERIFY2.ARO}

REFERENCE: Michel A. Saad, Compressible Fluid Flow, 2nd Edition, Prentice-Hall, Englewood Cliffs, NJ, 1993, Page 215, example 5.3

GAS: Methane

ASSUMPTIONS: 1) Adiabatic, 2) Perfect gas

RESULTS:

\begin{tabular}{|l|r|r|}
\hline \multicolumn{1}{|c|}{ Parameter } & \multicolumn{1}{c|}{ Saad } & AFT Arrow 2.0 \\
\hline Maximum pipe length $(\mathrm{m})$ & 4024 & 4029.49 \\
\hline$P_{2}-$ Static pressure at exit $(\mathrm{kPa})$ & 48.2 & 48.10 \\
\hline$V_{2}-$ Velocity at exit $(\mathrm{m} / \mathrm{s})$ & 433.17 & 433.13 \\
\hline$T_{2}-$ Static temperature at exit (deg. K) & 278.43 & 277.88 \\
\hline
\end{tabular}

\section{DISCUSSION:}

As specified, inlet conditions are known and the outlet conditions are sonic. The pipe length that yields sonic flow is the objective. With the known inlet conditions, an implied mass flow rate exists. To pose the problem in AFT Arrow terms, a few simple calculations are needed to obtain the mass flow rate. Once obtained, it is applied as a flow demand at the exit.

The problem states that the inlet velocity $V_{1}$ is $30 \mathrm{~m} / \mathrm{s}, P_{1}=0.8 \mathrm{MPa}, T_{1}=320 \mathrm{~K}$. From the ideal gas law, density, sonic speed and mass flow rate are:

$$
\begin{aligned}
& \rho_{1}=\frac{P_{1}}{R T_{1}}=\frac{0.8 \mathrm{MPa}}{\left(.5179 \frac{\mathrm{kJ}}{\mathrm{kg} \cdot \mathrm{K}}\right)(320 \mathrm{~K})}=4.823 \mathrm{~kg} / \mathrm{m}^{3} \\
& \dot{m}=\rho_{1} V_{1} A=\left(4.823 \frac{\mathrm{kg}}{\mathrm{m}^{3}}\right)\left(30 \frac{\mathrm{m}}{\mathrm{s}}\right)\left(\frac{\pi}{4} 0.3^{2} \mathrm{~m}^{2}\right)=10.2265 \mathrm{~kg} / \mathrm{s}
\end{aligned}
$$

AFT Arrow does not solve for pipe length. To obtain the maximum pipe length, different lengths must be guessed with lengths that exceed sonic flow discarded.

Note that the friction factor in Saad is the Fanning friction factor. To obtain the Darcy-Weisbach friction factor used in AFT Arrow, multiply the Fanning friction factor by 4.

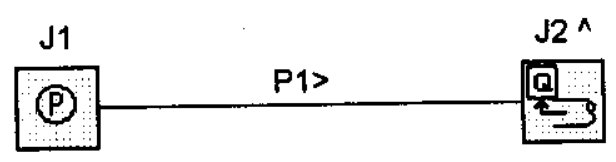

Copyright @ 1999 Applied Flow Technology Corp. All rights reserved.

* Printed with permission. 
Michel A. Saad

Compressible Fluid Flow, 2nd Edition, Prentice-Hall, Englewood Cliffs, NJ, 1993

page 215, example 5.3

Methane flow - Example assumes methane is a perfect gas and flow is adiabatic

See Verify2.doc MS Word file for comparison with published results

Title: Verify2.aro - Methane Example from Saad pp. 215-216

Number of Pipes= 1

Number of Junctions $=2$

Length March Solution Method with Mach Number Limits

Segments Per Pipe $=50$

Mach Number Increment= .01

Pressure Tolerance= .0001 relative change

Mass Flow Rate Tolerance $=.0001$ relative change

Enthalpy Tolerance $=.0001$ relative change

Concentration Tolerance $=.0001$ relative change

Flow Relaxation $=$ (Automatic)

Pressure Relaxation= (Automatic)

Fluid Database: AFT Standard

Fluid= Methane

Max Fluid Temperature Datar 400 deg. K

Min Fluid Temperature Data $=150$ deg. $K$

Molecular Weight $=16.043 \mathrm{amu}$

Gas Constant $=0.51790380 \mathrm{~kJ} / \mathrm{kg}-\mathrm{K}$

Critical Pressure $=4.5988 \mathrm{MPa}$

Critical Temperature $=190.555$ deg. $K$

Acentric Factor $=.008$

Equation of Statex Ideal Gas

Enthalpy Model= Reference

Specific Heat Ratio Accuracy= High

Atmospheric Pressure= $1 \mathrm{~atm}$

Gravitational Acceleration $=1 \mathrm{~g}$ 's

Standard Pressure $=14.696$ psia

Standard Temperature $=60$ deg. $F$

Turbulent Flow Above Reynolds Number $=\mathbf{4 0 0 0}$

Laminar Flow Below Reynolds Number $\mathbf{2 3 0 0}$

Specific Heat Ratio Accuracy= High

Atmospheric Pressure $=1 \mathrm{~atm}$

Gravitational Acceleration $=1 \mathrm{~g}$

Standard Pressure= 14.696 psia

Standard Temperature $=60 \mathrm{deg} . F$

Turbulent Flow Above Reynolds Number $=4000$

Laminar Flow Below Reynolds Number $=\mathbf{2 3 0 0}$

\section{Pipe Input Table}

\begin{tabular}{|c|r|r|r|r|r|r|r|r|r|r|}
\hline Pipe & Name & $\begin{array}{c}\text { Pipe } \\
\text { Defined }\end{array}$ & Length & $\begin{array}{c}\text { Length } \\
\text { Units }\end{array}$ & $\begin{array}{r}\text { Hydraulic } \\
\text { Diameter }\end{array}$ & $\begin{array}{c}\text { Hydraulic } \\
\text { Diam. Units }\end{array}$ & Roughness & $\begin{array}{c}\text { Roughness } \\
\text { Units }\end{array}$ & Losses (K) & Initial Flow \\
\hline 1 & Pipe & Yes & 4029.49 & meters & 0.3 & meters & 0.00015 & Eps/D & 0 & \\
\hline
\end{tabular}

\begin{tabular}{|c|c|c|c|c|c|c|c|}
\hline Pipe & $\begin{array}{c}\text { Initial Flow } \\
\text { Units }\end{array}$ & $\begin{array}{c}\text { Junctions } \\
\text { (Up,Down) }\end{array}$ & Geometry & Material & Size & Type & $\begin{array}{c}\text { Special } \\
\text { Condition }\end{array}$ \\
\hline 1 & & 1,2 & Cylindrical Pipe & Ungpecified & & & None \\
\hline
\end{tabular}

\section{Assigned Flow Table}


SNF-5331, Rev. 0

AFT Arrow 2.0 Input

(2)

12/7/99 10:13 AM

Numatec Hanford Corporation

Verify2.aro - Methane Example from Saad pp. 215-216

\begin{tabular}{|c|c|c|c|c|c|c|c|c|c|}
\hline Assigned Flow & Name & $\begin{array}{c}\text { Object } \\
\text { Defined }\end{array}$ & $\begin{array}{c}\text { Database } \\
\text { Source }\end{array}$ & $\begin{array}{c}\text { Special } \\
\text { Condition }\end{array}$ & Fluid & Type & Flow & $\begin{array}{c}\text { Flow } \\
\text { Units }\end{array}$ & Temperature \\
\hline 2 & Assigned Flow & Yes & & None & Methane & Outflow & 10.2265 & $\mathrm{~kg} / \mathrm{sec}$ & \\
\hline
\end{tabular}

\begin{tabular}{|c|r|r|}
\hline Assigned Flow & $\begin{array}{c}\text { Temperature } \\
\text { Units }\end{array}$ & $\begin{array}{c}\text { Loss } \\
\text { Model }\end{array}$ \\
\hline 2 & deg. K & 0 \\
\hline
\end{tabular}

\section{Assigned Pressure Table}

\begin{tabular}{|c|c|c|c|c|c|c|c|c|}
\hline Assigned Pressure & Name & $\begin{array}{l}\text { Object } \\
\text { Defined }\end{array}$ & $\begin{array}{c}\text { Database } \\
\text { Source }\end{array}$ & $\begin{array}{l}\text { Special } \\
\text { Condition }\end{array}$ & Fluid & Pressure & $\begin{array}{c}\text { Pressure } \\
\text { Units }\end{array}$ & Temperature \\
\hline 1 & Assigned Pressure & Yes & & N/A & Methane & 0.8 & $\mathrm{MPa}$ & 320 \\
\hline
\end{tabular}

\begin{tabular}{|c|c|r|}
\hline Assigned Pressure & $\begin{array}{c}\text { Temperature } \\
\text { Units }\end{array}$ & $\begin{array}{c}\text { Loss } \\
\text { Factor }\end{array}$ \\
\hline 1 & deg. K & 0 \\
\hline
\end{tabular}


Michel A. Saad

Compressible Fluid Flow, 2nd Edition, Prentice-Hall, Englewood Cliffs, NJ, 1993

page 215, example 5.3

Methane flow - Example assumes methane is a perfect gas and flow is adiabatic

See Verify2.doc MS Word file for comparison with published results

Title: Verify2.aro - Methane Example from Saad pp. 215-216

Analysis run on: $12 / 7 / 99$ 10:13:19 AM

Input File: C:LAFT ProductsLAFT ArrowlVerificationiverify2.aro

Execution Time $=0.83$ seconds

Total Number Of Pressure Iterations $=0$

Total Number Of Flow Iterations $=2$

Total Number Of Enthalpy Iterations $=\mathbf{2}$

Number Of Pipes $=1$

Number Of Junctions $=2$

Length March Solution Method with Mach Number Limits

Segments Per Pipe $=50$

Mach Number Increment $=.01$

Pressure Tolerance $=.0001$ relative change

Mass Flow Rate Tolerance $=.0001$ relative change

Enthalpy Tolerance $=.0001$ relative change

Flow Relaxation $=$ (Automatic)

Pressure Relaxation= (Automatic)

Fluid Database: AFT Standard

Fluid= Methane

Max Fluid Temperature Data $=400$ deg. $K$

Min Fluid Temperature Data $=150 \mathrm{deg} . \mathrm{K}$

Molecular Weight $=16.043 \mathrm{amu}$

Gas Constant $=0.51790380 \mathrm{~kJ} / \mathrm{kg}-\mathrm{K}$

Critical Pressure $=4.5988 \mathrm{MPa}$

Critical Temperature $=190.555 \mathrm{deg} . \mathrm{K}$

Acentric Factor $=.008$

Equation of State $=$ Ideal Gas

Enthalpy Model= Reference

Specific Heat Ratio Accuracy $=$ High

Atmospheric Pressure $=1 \mathrm{~atm}$

Gravitational Acceleration $=1 \mathrm{~g}$ 's

Standard Pressure $=14.696$ psia

Standard Temperature $=60$ deg. F

Turbulent Flow Above Reynolds Number $=4000$

Laminar Flow Below Reynolds Number $=2300$

Specific Heat Ratio Accuracy= High

Atmospheric Pressure $=1 \mathrm{~atm}$

Gravitational Acceleration $=1 \mathrm{~g}$

Standard Pressure $=14.696 \mathrm{psia}$

Standard Temperature $=60$ deg. $F$

Turbulent Flow Above Reynolds Number $=4000$

Laminar Flow Below Reynolds Number $=2300$

Overall Delta Pressure $=-0.71410561 \mathrm{MPa}$

Total Inflow $=10.226500 \mathrm{~kg} / \mathrm{sec}$

Total Outflow $=10.226500 \mathrm{~kg} / \mathrm{sec}$

Total Energy Inflow $=10,536.053 \mathrm{~kW}$

Total Energy Outflow $=10,536.053 \mathrm{~kW}$

Total Heat Transferred Into System $=0.0000000 \mathrm{~kW}$

Maximum Pressure is $0.80000001 \mathrm{MPa}$ at Junction 1 Inlet

Minimum Pressure is $0.048102446 \mathrm{MPa}$ at Junction 2 Inlet

Maximum Static Temperature is $48.621952 \mathrm{deg}$. $C$ at Junction 2 Inlet

Minimum Static Temperature is $47.045990 \mathrm{deg}$. $C$ at Junction 1 Inlet

Pipe Output Table 
SNF-5331, Rev. 0

Verify2.aro - Methane Example from Saad pp. 215-216

\begin{tabular}{|c|c|c|c|c|c|c|c|c|c|}
\hline Pipe & $\begin{array}{c}\text { Length } \\
\text { (meters) }\end{array}$ & $\begin{array}{c}\text { Mass Flow } \\
(\mathrm{kg} / \mathrm{sec})\end{array}$ & $\begin{array}{c}\text { Vel. In } \\
\text { (metera/sec) }\end{array}$ & $\begin{array}{c}\text { Vel. Out } \\
\text { (metera/sec) }\end{array}$ & Mach \# In & Mach \# Out & $\begin{array}{c}\text { P Static In } \\
\text { P Static } \\
\text { Out } \\
(\mathrm{kPa})\end{array}$ & $\begin{array}{c}\text { T Static In } \\
\text { (deg. K) }\end{array}$ \\
\hline 1 & 4029.4900 & 10.226500 & 29.991524 & 433.13260 & 0.064798974 & 0.99336141 & 800.00006 & 48.102448 & 320.00003 \\
\hline
\end{tabular}

\begin{tabular}{|c|c|}
\hline Pipe & $\begin{array}{c}\text { T Static } \\
\text { Out } \\
\text { (deg. K) }\end{array}$ \\
\hline 1 & 277.87515 \\
\hline
\end{tabular}

\section{All Junction Table}

\begin{tabular}{|c|c|c|c|c|}
\hline Jet & $\begin{array}{c}\text { P Static In } \\
(\mathrm{KPa})\end{array}$ & $\begin{array}{c}\text { P Static Out } \\
(\mathrm{kPa})\end{array}$ & $\begin{array}{c}\text { T Static In } \\
(\text { deg. K) }\end{array}$ & $\begin{array}{c}\text { T Static Out } \\
\text { (deg. F) }\end{array}$ \\
\hline 1 & 800.000061 & 800.000061 & 320.00000 & 116.330002 \\
\hline 2 & 48.102448 & 48.102448 & 277.87512 & 40.505234 \\
\hline
\end{tabular}


TITLE: VERIFY3.ARO

REFERENCE: Crane Co., Flow of Fluids Through Valves, Fittings, and Pipe, Technical Paper No. 410, Crane Co., Joliet, IL, 1988, Page 4-6, example 4-10

GAS: Steam

ASSUMPTIONS: Example does not specify the heat transfer conditions, so it was assumed adiabatic in this model.

RESULTS:

\begin{tabular}{|c|r|r|}
\hline Parameter & Crane & AFT Arrow 2.0 \\
\hline Static pressure drop (psi) & 40.1 & 41.59 \\
\hline
\end{tabular}

\section{DISCUSSION:}

Crane does not make a distinction between static and stagnation pressure, and it appears that static pressure is usually assumed. Therefore, the inlet pressure of $600 \mathrm{psig}$ was assumed to be static pressure.

To make a one-to-one comparison, the $\mathrm{K}$ factors and friction factor used in Crane was used directly in AFT Arrow. The $\mathrm{K}$ factors were modeled as Additional Losses, which evenly spreads the effect of resistance across the entire pipe. In practice, the velocity changes in the pipe can yield different answers for fitting pressure losses depending on where they are actually located.

The AFT Arrow model uses the Redlich-Kwong real gas equation of state for steam. Note that the Crane formula underpredicts the pressure drop (by about $4 \%$ ), which in most applications is not conservative.

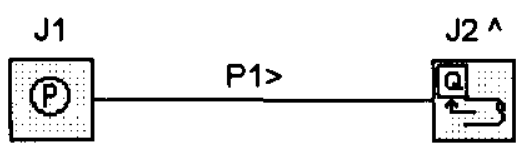

Copyright $\bigcirc 1999$ Applied Flow Technology Corp. All rights reserved. 
SNF-5331, Rev. 0

AFT Arrow 2.0 Input

Crane Co.

Flow of Fluids Through Valves, Fittings, and Pipe, Technical Paper No. 410, Crane Co., Joliet, IL, 1988

Page 4-6, example 4-10

Steam flow - Example does not specify the heat transfer conditions, so it was assumed adiabatic in this model

See Verify3.doc MS Word file for comparison with published results

Title: Verify3.aro - Steam flow in a pipe, Example 4-10 from Crane page 4-6

Number Of Pipes $=1$

Number Of Junctions $=2$

Length March Solution Method with Mach Number Limits

Segments Per Pipe $=10$

Mach Number Increment $=.01$

Pressure Tolerance $=.0001$ relative change

Mass Flow Rate Tolerance $=.0001$ relative change

Enthalpy Tolerance $=0001$ relative change

Flow Relaxation $=.5$

Pressure Relaxation $=.5$

Fluid Database: AFT Standard

Fluid= Steam (Simplified)

Max Fluid Temperature Data $=1500$ deg. $F$

Min Fluid Temperature Data $=200$ deg. F

Molecular Weight $=18.016 \mathrm{amu}$

Gas Constant $=0.1102 \mathrm{Btu} / \mathrm{bm}-\mathrm{R}$

Critical Pressure $=3208.22$ psia

Critical Temperature $=1165.09$ deg. $R$

Acentric Factor $=.344$

Equation of State $=$ Redlich $-\mathrm{K}$ wong

Enthalpy Model= Generalized

Specific Heat Ratio Accuracy= High

Atmospheric Pressure= $1 \mathrm{~atm}$

Gravitational Acceleration $=1 \mathrm{~g}$ 's

Standard Pressure $=14.696$ psia

Standard Temperature $=60 \mathrm{deg} . F$

Turbulent Flow Above Reynolds Number $=4000$

Laminar Flow Below Reynolds Number $=2300$

Specific Heat Ratio Accuracy $=$ High

Atmospheric Pressure $=1 \mathrm{~atm}$

Gravitational Acceleration $=1 \mathrm{~g}$

Standard Pressure $=14.696$ psia

Standard Temperature $=60 \mathrm{deg} . F$

Turbulent Flow Above Reynolds Number $=4000$

Laminar Flow Below Reynolds Number $=\mathbf{2 3 0 0}$

\section{Pipe Input Table}

\begin{tabular}{|c|r|r|r|r|r|r|r|r|r|r|}
\hline Pipe & Name & $\begin{array}{c}\text { Pipe } \\
\text { Defined }\end{array}$ & Length & $\begin{array}{c}\text { Length } \\
\text { Units }\end{array}$ & $\begin{array}{c}\text { Hydraulic } \\
\text { Diameter }\end{array}$ & $\begin{array}{c}\text { Hydraulic } \\
\text { Diam. Units }\end{array}$ & Roughness & $\begin{array}{c}\text { Roughness } \\
\text { Units }\end{array}$ & Losses (K) & Initial Flow \\
\hline 1 & Pipe & Yes & 400 & feet & 5.761 & inches & 0.015 & Explicit f & 3.5 & \\
\hline
\end{tabular}

\begin{tabular}{|c|c|r|c|r|c|c|c|}
\hline Pipe & $\begin{array}{c}\text { Initial Flow } \\
\text { Units }\end{array}$ & $\begin{array}{c}\text { Junctions } \\
\text { (Up,Down) }\end{array}$ & Geometry & Material & Size & Type & $\begin{array}{c}\text { Special } \\
\text { Condition }\end{array}$ \\
\hline 1 & & 1,2 & Cylindrical Pipe & Steel & 6 inch & schedule 80 & None \\
\hline
\end{tabular}

\section{Assigned Flow Table}


SNF-5331, Rev. 0

AFT Arrow 2.0 Input Numatec Hanford Corporation
(2)

$12 / / 9910: 08 \mathrm{AM}$

Verify 3.aro - Steam flow in a pipe, Example 4-10 from Crane page 4-6

\begin{tabular}{|c|r|r|}
\hline Assigned Flow & $\begin{array}{c}\text { Temperature } \\
\text { Units }\end{array}$ & $\begin{array}{c}\text { Loss } \\
\text { Model }\end{array}$ \\
\hline 2 & deg. F & 0 \\
\hline
\end{tabular}

Assigned Pressure Table

\begin{tabular}{|c|c|r|r|r|r|r|r|}
\hline Assigned Pressure & Name & $\begin{array}{c}\text { Object } \\
\text { Defined }\end{array}$ & $\begin{array}{c}\text { Database } \\
\text { Source }\end{array}$ & $\begin{array}{c}\text { Special } \\
\text { Condition }\end{array}$ & Fluid & $\begin{array}{r}\text { Pressure } \\
\text { Pressure } \\
\text { Units }\end{array}$ & \begin{tabular}{c} 
Temperature \\
\hline 1
\end{tabular} \\
\hline
\end{tabular}

\begin{tabular}{|c|r|r|}
\hline Assigned Pressure & $\begin{array}{c}\text { Temperature } \\
\text { Units }\end{array}$ & $\begin{array}{c}\text { Loss } \\
\text { Factor }\end{array}$ \\
\hline 1 & deg. F & 0 \\
\hline
\end{tabular}

35 
Crane Co.

Flow of Fluids Through Valves, Fittings, and Pipe, Technical Paper No. 410, Crane Co., Joliet, IL, 1988

Page 4-6, example 4-10

Steam flow - Example does not specify the heat transfer conditions, 80 it was assumed adiabatic in this model

See Verify3.doc MS Word file for comparison with published results

Title: Verify3.aro - Steam flow in a pipe, Example 4-10 from Crane page 4-6

Analysis run on: 12/7/99 10:04:11 AM

Input File: C:MFT ProductsLAFT ArrowlVerificationiverify3.aro

Execution Time $=0.39$ seconds

Total Number Of Pressure lterations $=0$

Total Number Of Flow Iterations $=2$

Total Number of Enthalpy Iterations= 2

Number Of Pipes $=1$

Number Of Junctions= 2

Length March Solution Method with Mach Number Limits

Segments Per Pipe $=10$

Mach Number Increment= 01

Pressure Tolerance $=.0001$ relative change

Mass Flow Rate Tolerance $=.0001$ relative change

Enthalpy Tolerance $=.0001$ relative change

Flow Relaxation $=.5$

Pressure Relaxation $=.5$

Fluid Database: AFT Standard

Fluid= Steam (Simplified)

Max Fluid Temperature Data $=1500 \mathrm{deg} . F$

Min Fluid Temperature Data $=200$ deg. F

Molecular Weight= $18.016 \mathrm{amu}$

Gas Constant $=0.1102$ Btu/bm-R

Critical Pressure $=3208.22$ psia

Critical Temperature $=1165.09 \mathrm{deg} . \mathrm{R}$

Acentric Factor $=.344$

Equation of State $=$ Redlich-Kwong

Enthalpy Model= Generalized

Specific Heat Ratio Accuracy= High

Atmospheric Pressure $=1 \mathrm{~atm}$

Gravitational Acceleration $=1 \mathrm{~g}$ 's

Standard Pressure $=14.696$ psia

Standard Temperature $=60 \mathrm{deg}$. F

Turbulent Flow Above Reynolds Number $=4000$

Laminar Flow Below Reynolds Number $\mathbf{2 3 0 0}$

Specific Heat Ratio Accuracy $=$ High

Atmospheric Pressure $=1 \mathrm{~atm}$

Gravitational Acceleration $=1 \mathrm{~g}$

Standard Pressure $=14.696$ psia

Standerd Temperature $=60$ deg. $F$

Turbulent Flow Above Reynolds Number 4000

Laminar Flow Below Reynolds Number $=2300$

Overall Delta Pressure $=-41.41$ psia

Total Intlow $=25.00 \mathrm{lbm} / 8 e \mathrm{c}$

Total Outflow $=25.00 \mathrm{lbm} / \mathrm{sec}$

Total Energy Inflow= 35,663 Btu/s

Total Energy Outflow= 35,663 Btu/s

Total Heat Transferred Into System $=0.000 \mathrm{Btu} / \mathrm{s}$

Maximum Pressure is 614.7 psia at Junction 1 Inlet Minimum Pressure is 573.1 psia at Junction 2 Inlet

Maximum Static Temperature is 851.0 deg. F at Junction 1 Inlet

Minimum Static Temperature is $848.0 \mathrm{deg}$. F at Junction 2 Inlet 
SNF-5331, Rev. 0

Verify3.aro - Steam flow in a pipe, Example 4-10 from Crane page 4-6

\begin{tabular}{|c|c|c|c|c|c|c|c|c|c|c|c|c|}
\hline Pipe & $\begin{array}{c}\text { dP Static } \\
\text { Total } \\
\text { (psid) } \\
\end{array}$ & $\begin{array}{c}\text { Mass } \\
\text { Flow } \\
(\mathrm{lbm} / \mathrm{hr})\end{array}$ & $\begin{array}{c}\text { Vel. } \\
\text { In } \\
\text { (feet/sec) }\end{array}$ & $\begin{array}{c}\text { Vel. } \\
\text { Out } \\
\text { (feet/sec) }\end{array}$ & $\begin{array}{l}\text { P Static } \\
\text { In } \\
\text { (paig) }\end{array}$ & $\begin{array}{c}\text { P Static } \\
\text { Out } \\
\text { (psia) }\end{array}$ & $\begin{array}{l}\text { T Static } \\
\text { In } \\
\text { (deg. F) }\end{array}$ & $\begin{array}{c}\text { T Static } \\
\text { Out } \\
\text { (deg. F) }\end{array}$ & $f$ & K & fL/D & $\begin{array}{c}f \cup \\
D+K\end{array}$ \\
\hline 1 & 41.59 & 90,000 & 167.0 & 179.2 & 600.0 & 558.4 & 850.0 & 846.8 & 0.01500 & 3.500 & 12.50 & 16.00 \\
\hline
\end{tabular}

\section{All Junction Table}

\begin{tabular}{|c|c|c|c|c|}
\hline Jet & $\begin{array}{c}\text { P Static } \\
\text { In } \\
\text { (psia) }\end{array}$ & $\begin{array}{c}\text { P Static } \\
\text { Out } \\
\text { (psia) }\end{array}$ & $\begin{array}{c}\text { T Static } \\
\text { In } \\
\text { (deg. F) }\end{array}$ & $\begin{array}{c}\text { T Static } \\
\text { Out } \\
\text { (deg. F) }\end{array}$ \\
\hline 1 & 614.7 & 614.7 & 850.0 & 850.0 \\
\hline 2 & 573.1 & 573.1 & 846.8 & 846.8 \\
\hline
\end{tabular}


TITLE: VERIFY4.ARO

REFERENCE: Crane Co., Flow of Fluids Through Valves, Fittings, and Pipe, Technical Paper No. 410, Crane Co., Joliet, IL, 1988, Page 4-9, example 4-16

GAS: Air

ASSUMPTIONS: Example does not specify the heat transfer conditions, so it was assumed isothermal in this model.

RESULTS:

\begin{tabular}{|c|c|c|}
\hline S & Crane & AFT Arrow 2.0 \\
\hline Static pressure drop (psi) & 2.61 & 2.712 \\
\hline Volumetric flow rate at inlet ( $\mathrm{ft} 3 / \mathrm{min})$ & 20.2 & 20.21 \\
\hline Volumetric flow rate at outlet ( $\mathrm{ft} 3 / \mathrm{min})$ & 20.9 & 20.93 \\
\hline Velocity inlet (ft/min) & 3,367 & 3,368 \\
\hline Velocity inlet (ft/min) & 3,483 & 3,487 \\
\hline
\end{tabular}

\section{DISCUSSION:}

Crane does not make a distinction between static and stagnation pressure, and it appears that static pressure is usually assumed. Therefore, the inlet pressure of $65 \mathrm{psig}$ was assumed to be static pressure.

Note that the Crane formula underpredicts the pressure drop (by about $4 \%$ ), which in most applications is not conservative.

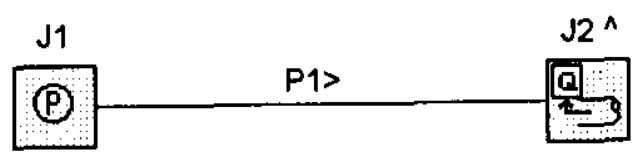

Copyright 1999 Applied Flow Technology Corp. All rights reserved. 
AFT Arrow 2.0 Input

Numatec Hanford Corporation
(1)

12/7/99 10:24 AM

Verify4.aro - Air flow in a pipe, Example 4-16 from Crane page 4-9

Crane Co.

Flow of Fluids Through Valves, Fittings, and Pipe, Technical Paper No. 410, Crane Co., Joliet, IL, 1988

Page 4-9, example 4-16

Air flow - Example does not specify the heat transfer conditions, but it appears to use isothermal so this model uses isothermal

See Verify4.doc MS Word file for comparison with published results

Title: Verify4.aro - Air flow in a pipe, Example 4-16 from Crane page 4-9

Number Of Pipes $=1$

Number Of Junctions $=2$

Length March Solution Method with Mach Number Limits

Segments Per Pipe $=2$

Mach Number Increment $=.01$

Pressure Tolerance $=.0001$ relative change

Mass Flow Rate Tolerance $=.0001$ relative change

Enthalpy Tolerance $=.0001$ relative change

Concentration Tolerance $=.0001$ relative change

Flow Relaxation $=$ (Automatic)

Pressure Relaxation= (Automatic)

Fluid Database: AFT Standard

Fluid = Air

Max Fluid Temperature Data $=1000$ deg. $K$

Min Fluid Temperature Data $=200$ deg. K

Molecular Weight $=28.97 \mathrm{amu}$

Gas Constant $=0.06855 \mathrm{Btu} / \mathrm{bm}-\mathrm{R}$

Critical Pressure $=37.25$ atm

Critical Temperature $=132.41$ deg. $\mathrm{K}$

Acentric Factor $=.021$

Equation of State $=$ Ideal Gas

Enthalpy Model= Reference

Specific Heat Ratio Accuracy $=$ High

Atmospheric Pressure $=1 \mathrm{~atm}$

Gravitational Acceleration $=1 \mathrm{~g}$ 's

Standard Pressure $=14.696 \mathrm{psia}$

Standard Temperature $=60$ deg. F

Turbulent Flow Above Reynolds Number $=4000$

Laminar Flow Below Reynolds Number $=\mathbf{2 3 0 0}$

Specific Heat Ratio Accuracy= High

Atmospheric Pressure $=1 \mathrm{~atm}$

Gravitational Acceleration $=1 \mathrm{~g}$

Standard Pressure $=14.696 \mathrm{psia}$

Standard Temperature $=60$ deg. $F$

Turbulent Flow Above Reynolds Number $=4000$

Laminar Flow Below Reynolds Number $=\mathbf{2 3 0 0}$

\section{Pipe Input Table}

\begin{tabular}{|r|r|r|r|r|r|r|r|r|r|r|}
\hline Pipe & Name & $\begin{array}{c}\text { Pipe } \\
\text { Defined }\end{array}$ & Length & $\begin{array}{r}\text { Length } \\
\text { Units }\end{array}$ & $\begin{array}{r}\text { Hydraulic } \\
\text { Diameter }\end{array}$ & $\begin{array}{c}\text { Hydraulic } \\
\text { Diam. Units }\end{array}$ & Roughness & $\begin{array}{c}\text { Roughness } \\
\text { Units }\end{array}$ & Losses (K) & Initial Flow \\
\hline 1 & Pipe & Yes & 75 & feet & 1.049 & inches & 0.00015 & feet & 0 & \\
\hline
\end{tabular}

\begin{tabular}{|c|r|r|c|r|c|c|c|}
\hline Pipe & $\begin{array}{c}\text { Initial Flow } \\
\text { Units }\end{array}$ & $\begin{array}{c}\text { Junctions } \\
\text { (Up,Down) }\end{array}$ & Geometry & Material & Size & Type & $\begin{array}{c}\text { Special } \\
\text { Condition }\end{array}$ \\
\hline 1 & & 1,2 & Cylindrical Pipe & Steel & 1 inch & schedule 40 & None \\
\hline
\end{tabular}

\section{Assigned Flow Table}


SNF-5331, Rev. 0

AFT Arrow 2.0 Input

12//99 10:24 AM

Numatec Hanford Corporation

Verify4.aro - Air flow in a pipe, Example 4-16 from Crane page 4-9

\begin{tabular}{|c|r|r|r|r|r|r|r|r|r|r|}
\hline Assigned Flow & Name & $\begin{array}{c}\text { Object } \\
\text { Defined }\end{array}$ & $\begin{array}{c}\text { Database } \\
\text { Source }\end{array}$ & $\begin{array}{c}\text { Special } \\
\text { Condition }\end{array}$ & Fluid & Type & Flow & $\begin{array}{c}\text { Flow } \\
\text { Units }\end{array}$ & $\begin{array}{c}\text { Temperature } \\
\text { Temperature } \\
\text { Units }\end{array}$ \\
\hline 2 & Assigned Flow & Yes & & None & Air & Outflow & 100 & scfm & 110 & deg. F \\
\hline
\end{tabular}

\begin{tabular}{|c|r|}
\hline Assigned Flow & $\begin{array}{c}\text { Loss } \\
\text { Model }\end{array}$ \\
\hline 2 & 0 \\
\hline
\end{tabular}

Assigned Pressure Table

\begin{tabular}{|c|c|r|r|r|r|r|r|r|}
\hline Assigned Pressure & Name & $\begin{array}{c}\text { Object } \\
\text { Defined }\end{array}$ & $\begin{array}{c}\text { Database } \\
\text { Source }\end{array}$ & $\begin{array}{c}\text { Special } \\
\text { Condition }\end{array}$ & Fluid & Pressure & $\begin{array}{c}\text { Pressure } \\
\text { Units }\end{array}$ & \begin{tabular}{c} 
Temperature \\
\hline 1
\end{tabular} \\
\hline
\end{tabular}

\begin{tabular}{|c|c|c|}
\hline Assigned Pressure & $\begin{array}{c}\text { Temperature } \\
\text { Units }\end{array}$ & $\begin{array}{c}\text { Loss } \\
\text { Factor }\end{array}$ \\
\hline 1 & deg. $F$ & 0 \\
\hline
\end{tabular}


Crane Co.

Flow of Fluids Through Valves, Fittings, and Pipe, Technical Paper No. 410, Crane Co., Joliet, IL, 1988

Page 4-9, example 4-16

Air flow - Example does not specify the heat transfer conditions, but it appears to use isothermal so this model uses isothermal

See Verify4.doc MS Word file for comparison with published results

Title: Verify4.aro - Air flow in a pipe, Example 4-16 from Crane page 4-9

Analysis run on: $12 / 7 / 99$ 10:22:33 AM

Input File: C:LAFT ProductsLAFT ArrowlVerificationlverify4.aro

Execution Time $=0.17$ seconds

Total Number Of Pressure Iterations $=0$

Total Number Of Flow lterations= 2

Total Number Of Enthalpy Iterations $=2$

Number Of Pipes $=1$

Number of Junctions $=2$

Length March Solution Method with Mach Number Limits

Segments Per Pipe= 2

Mach Number Increment= .01

Pressure Tolerance $=.0001$ relative change

Mass Flow Rate Tolerance $=.0001$ relative change

Enthalpy Tolerance $=.0001$ relative change

Flow Relaxation= (Automatic)

Pressure Relaxation= (Automatic)

Fluid Database: AFT Standard

Fluid= Air

Max Fluid Temperature Data $=1000$ deg. K

Min Fluid Temperature Data $\mathbf{2 0 0}$ deg. K

Molecular Weight $=28.97 \mathrm{amu}$

Gas Constant $=0.06855$ Btu/bm-R

Critical Pressure $=37.25 \mathrm{~atm}$

Critical Temperature $=132.41 \mathrm{deg} . \mathrm{K}$

Acentric Factor $=.021$

Equation of State $=$ Ideal Gas

Enthalpy Model= Reference

Specific Heat Ratio Accuracy= High

Atmospheric Pressure= $1 \mathrm{~atm}$

Gravitational Acceleration $=1 \mathrm{~g}$ 's

Standard Pressure= 14.696 psia

Standard Temperature $=60 \mathrm{deg} . F$

Turbulent Flow Above Reynolds Number $=4000$

Laminar Flow Below Reynolds Number $=\mathbf{2 3 0 0}$

Specific Heat Ratio Accuracy $=$ High

Atmospheric Pressure $=1 \mathrm{~atm}$

Gravitational Acceleration $=1 \mathrm{~g}$

Standard Pressure $=14.696$ psia

Standard Temperature $=60$ deg. $F$

Turbulent Flow Above Reynolds Number $=4000$

Laminar Flow Below Reynolds Number $=2300$

Overall Delta Pressure $=-\mathbf{2 . 7 0 7}$ psia

Total Inflow: $0.1272 \mathrm{lbm} / \mathrm{sec}$

Total Outflow $=0.1272 \mathrm{lbm} / \mathrm{sec}$

Total Energy Inflow $=26.10 \mathrm{Btu} / \mathrm{s}$

Total Energy Outflow= 26.10 Btu/s

Total Heat Transferred Into System $=5.740 E-04$ Btu/s

Maximum Pressure is 79.70 psia at Junction 1 Inlet

Minimum Pressure is 76.98 psia at Junction 2 Inlet

Maximum Static Temperature is $110.3 \mathrm{deg} . F$ at Junction 2 Inlet

Minimum Static Temperature is $110.3 \mathrm{deg}$. F at Junction 1 Inlet

\section{Pipe Output Table}


Verify4.aro - Air flow in a pipe, Example 4-16 from Crane page 4-9

\begin{tabular}{|c|c|c|c|c|c|c|c|c|c|c|}
\hline Pipe & $\begin{array}{c}\text { dP Static } \\
\text { Total } \\
\text { (psid) }\end{array}$ & $\begin{array}{c}\text { Vol. } \\
\text { Flow In } \\
\text { (ft3/min) }\end{array}$ & $\begin{array}{c}\text { Vol. } \\
\text { Flow Out } \\
\text { (ft3/min) }\end{array}$ & $\begin{array}{c}\text { Vel. } \\
\text { In } \\
\text { (feet/min) }\end{array}$ & $\begin{array}{c}\text { Vel. } \\
\text { Out } \\
\text { (feet/min) }\end{array}$ & $\begin{array}{c}\text { Mass } \\
\text { Flow } \\
\text { (scfm) }\end{array}$ & $\begin{array}{c}\text { P Static } \\
\text { In } \\
\text { (psig) }\end{array}$ & $\begin{array}{c}\text { P Static } \\
\text { Out } \\
\text { (psig) }\end{array}$ & $\begin{array}{c}\text { T Static } \\
\text { In } \\
\text { (deg. F) }\end{array}$ & $\begin{array}{c}\text { T Static } \\
\text { Out } \\
\text { (deg. F) }\end{array}$ \\
\hline 1 & 2.712 & 20.21 & 20.93 & 3368. & 3487. & 100.0 & 65.00 & 62.29 & 110.0 & 110.0 \\
\hline
\end{tabular}

\section{All Junction Table}

\begin{tabular}{|c|c|c|r|r|}
\hline Jet & $\begin{array}{c}\text { P Static } \\
\text { In } \\
\text { (psig) }\end{array}$ & $\begin{array}{c}\text { P Static } \\
\text { Out } \\
\text { (psig) }\end{array}$ & $\begin{array}{c}\text { T Static } \\
\text { in } \\
\text { (deg. F) }\end{array}$ & $\begin{array}{c}\text { T Static } \\
\text { Out } \\
\text { (deg. F) }\end{array}$ \\
\hline 1 & 65.00 & 65.00 & 110.0 & 110.0 \\
\hline 2 & 62.29 & 62.29 & 110.0 & 110.0 \\
\hline
\end{tabular}


TITLE: VERIFY5.ARO

REFERENCE: Crane Co., Flow of Fluids Through Valves, Fittings, and Pipe, Technical Paper No. 410 , Crane Co., Joliet, IL, 1988, Page 4-11, example 4-18

GAS: Natural Gas (mole fractions: $75 \%$ methane, $21 \%$ ethane, and $4 \%$ propane)

ASSUMPTIONS: Isothermal flow at 40 degrees $\mathrm{F}$

RESULTS:

\begin{tabular}{|l|r|r|}
\hline \multicolumn{1}{|c|}{ Parameter } & Crane & AFT Arrow 2.0 \\
\hline Mass flow rate using standard friction (MMscfd) $\dagger$ & 107.8 & 123.1 \\
\hline Mass flow rate using Weymouth (MMscfd) & 105.1 & $120.0^{*}$ \\
\hline Mass flow rate using Panhandle (MMscfd) & 133.7 & $118.5^{*}$ \\
\hline
\end{tabular}

† Crane's calculation uses the "Simplified Compressible Flow Formula"

* Crane uses the actual Weymouth and Panhandle equations. AFT Arrow does have these equations, but instead solves the governing mass and momentum equations over pipe sections. The AFT Arrow Weymouth and Panhandle solutions above were obtained using the Weymouth and Panhandle friction factor correlation options in AFT Arrow rather than the standard Darcy-Weisbach friction factor (as used in the first case above).

\section{DISCUSSION:}

The mixture properties for this example offer an opportunity to use the Chempak mixture capabilities. The problem statement does not say whether the fractions are on a mass or mole basis, but it does say in Crane that the mixture molecular weight is 20.1. This is consistent with mole fraction. AFT Arrow's output indicates the molecular weight of the mixture is 20.11 .

As noted above $\left({ }^{*}\right)$, AFT Arrow has optional friction factor models for Weymouth and Panhandle (see Crane, page 1-8 or AFT Arrow documentation). These were used for the second and third cases above. However, regardless of what friction factor model is used, AFT Arrow differs from any of the three methods above in that it directly solves the governing equations and it does so over pipe segments. In this model, the pipe was broken into 100 segments.

Since it can be easily demonstrated that AFT Arrow's solution satisfies the mass and momentum equations for this pipe, and the solution differs from the Crane solutions, the Crane solutions do not offer a mass and/or momentum balance.

Copyright $\mathbb{C} 1999$ Applied Flow Technology Corp. All rights reserved. 
TITLE: VERIFY6.ARO

REFERENCE: Crane Co., Flow of Fluids Through Valves, Fittings, and Pipe, Technical Paper No. 410 , Crane Co., Joliet, IL, 1988, Page 4-13, example 4-20

GAS: Steam

ASSUMPTIONS: Example does not specify the heat transfer conditions, but it appears from the calculation procedure that adiabatic flow is assumed. The AFT Arrow model assumes adiabatic.

RESULTS:

\begin{tabular}{|l|r|r|r|}
\hline \multicolumn{1}{|c|}{ Parameter } & $\begin{array}{c}\text { Crane Modified } \\
\text { Darcy Formula }\end{array}$ & $\begin{array}{c}\text { Crane Sonic } \\
\text { Velocity Formula }\end{array}$ & AFT Arrow 2.0 \\
\hline Mass flow rate (lbm/hr) & 11,780 & 11,180 & 11,042 \\
\hline Exit static enthalpy (But/lbm) & N/A & 1,196 & $1,147.6$ \\
\hline Exit static temperature (deg. F) & N/A & 317 & 240.7 \\
\hline
\end{tabular}

\section{DISCUSSION:}

Crane does not make a distinction between static and stagnation pressure, and it appears that static pressure is usually assumed. However, the problem statement is that the source steam comes from a header, where conditions are more likely stagnation. Therefore, stagnation pressure was assumed in the AFT Arrow model.

The Crane Sonic Velocity Formula yields a flow rate prediction that agrees well with the AFT Arrow prediction. The Crane Modified Darcy Formula appears to yield flow rates that are too high. If the inlet conditions in the AFT Arrow model are changed to static, the AFT Arrow flow rate prediction increases slightly to $11,359(\mathrm{lbm} / \mathrm{hr})$. AFT Arrow uses real gas properties for the steam as specified in the System Properties window.

The Crane sonic calculation assumes an isenthalpic process, which is why the exit static enthalpy is assumed to be constant at $1,196 \mathrm{Btu} / \mathrm{lbm}$. With this assumption, the exit temperature is $317 \mathrm{deg} . \mathrm{F}$.

However, an isenthalpic assumption turns out to be poor when the Energy Equation is applied. From the First Law,

$$
\dot{q}=\dot{m}\left(h_{1}+\frac{V_{1}^{2}}{2}-h_{2}-\frac{V_{2}^{2}}{2}\right)
$$

If the process is adiabatic, the heat transfer is zero and therefore,

$$
h_{2}=h_{1}+\frac{V_{1}^{2}}{2}-\frac{V_{2}^{2}}{2}
$$

Thus, the static enthalpy will drop because of the velocity increase. With this is accounted for, the exit static enthalpy decreases to 1148 . This yields an exit static temperature of $240.7 \mathrm{deg}$. F, which is 76 degrees cooler.

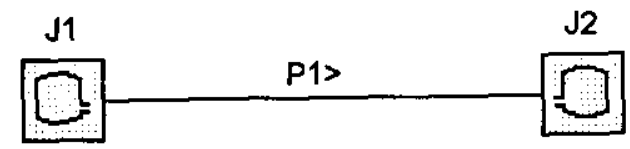

Copyright $\mathcal{O} 1999$ Applied Flow Technology Corp. All rights reserved.

* Printed with permission. 
Crane Co.

Flow of Fluids Through Valves, Fittings, and Pipe, Technical Paper No. 410, Crane Co., Joliet, IL, 1988

Page 4-13, example 4-20

Sonic choking comparison

Steam flow - Example assumes adiabatic flow

See Verify6.doc MS Word file for comparison with published results

Title: Verify6.aro - Steam flow in a pipe, Example 4-20 from Crane page 4-13

Number Of Pipes= 1

Number Of Junctions= 2

Length March Solution Method with Mach Number Limits

Segments Per Pipex 10

Mach Number Increment= .01

Pressure Tolerance $=0001$ relative change

Mass Flow Rate Tolerances .0001 relative change

Enthalpy Tolerances .0001 relative change

Flow Relaxation= .5

Pressure Relaxation $=.5$

Fluid Database: AFT Standard

Fluid= Steam (Simplified)

Max Fluid Temperature Data $=1500 \mathrm{deg} . \mathrm{F}$

Min Fluid Temperature Data $=\mathbf{2 0 0}$ deg. F

Molecular Weight= $18.016 \mathrm{amu}$

Gas Constant= 0.110227 Btu/lbm-R

Critical Presesure $=3208.22$ psia

Critical Temperature= 1165.09 deg. $R$

Acentric Factora .344

Equation of State= Redlich-Kwong

Enthalpy Model= Generalized

Specific Heat Ratio Accuracy= High

Atmospheric Pressure= $1 \mathrm{~atm}$

Gravitational Acceleration $=1 \mathrm{~g} / \mathrm{s}$

Standard Pressure= $14.696 \mathrm{psia}$

Standard Temperature $=60 \mathrm{deg} . F$

Turbulent Flow Above Reynolds Number $=4000$

Laminar Flow Below Reynolds Number $=\mathbf{2 3 0 0}$

Specific Heat Ratio Accuracy= High

Atmospheric Pressure= 1 atm

Gravitational Acceleration $=1 \mathrm{~g}$

Standard Pressure $=14.696$ psia

Standard Temperature $=60 \mathrm{deg} . F$

Turbulent Flow Above Reynolds Number $=\mathbf{4 0 0 0}$

Laminar Flow Below Reynolds Number $=2300$

\section{Pipe input Table}

\begin{tabular}{|c|r|r|r|r|r|r|r|r|r|r|}
\hline Pipe & Name & $\begin{array}{c}\text { Pipe } \\
\text { Defined }\end{array}$ & Length & $\begin{array}{r}\text { Length } \\
\text { Units }\end{array}$ & $\begin{array}{r}\text { Hydraulic } \\
\text { Diameter }\end{array}$ & $\begin{array}{c}\text { Hydraulic } \\
\text { Diam. Units }\end{array}$ & Roughness & $\begin{array}{c}\text { Roughness } \\
\text { Units }\end{array}$ & Losses (K) & Initial Flow \\
\hline 1 & Pipe & Yes & 30 & feet & 2.067 & inches & 0.00015 & feet & 8.53 & \\
\hline
\end{tabular}

\begin{tabular}{|c|c|c|c|c|c|c|c|}
\hline Pipe & $\begin{array}{c}\text { Initial Flow } \\
\text { Units }\end{array}$ & $\begin{array}{c}\text { Junctions } \\
\text { (Up,Down) }\end{array}$ & Geometry & Material & Size & Type & $\begin{array}{c}\text { Special } \\
\text { Condition }\end{array}$ \\
\hline 1 & & 1,2 & Cylindrical Pipe & Steel & $\mathbf{2}$ inch & schedule 40 & None \\
\hline
\end{tabular}

\section{Tank Table}


SNPF-S331, Rov. 0

AFT Arrow 2.0 Input

(2)

$127 / 99$ 11:01 AM

Numatec Hanford Corporation

Verify6.aro - Steam flow in a pipe, Example 4-20 from Crane page 4-13

\begin{tabular}{|c|c|c|c|c|c|c|c|c|c|c|}
\hline Tank & Name & $\begin{array}{l}\text { Object } \\
\text { Defined }\end{array}$ & $\begin{array}{c}\text { Database } \\
\text { Source }\end{array}$ & $\begin{array}{l}\text { Special } \\
\text { Condition }\end{array}$ & Fluid & Pressure & $\begin{array}{c}\text { Pressure } \\
\text { Units }\end{array}$ & Temperature & $\begin{array}{c}\text { Temperature } \\
\text { Units }\end{array}$ & $\begin{array}{l}\text { Balance } \\
\text { Energy }\end{array}$ \\
\hline 1 & Tank & Yes & & N/A & Steam (Simplified) & 170 & psia & 368.48 & $\operatorname{deg} . F$ & No \\
\hline 2 & Tank & Yes & & N/A & Steam (Simplified) & 14.7 & psia & 368.48 & $\operatorname{deg} . F$ & No \\
\hline
\end{tabular}

\begin{tabular}{|c|c|c|c|c|c|c|c|c|c|}
\hline Tank & $\begin{array}{c}\text { Balance } \\
\text { Concentration }\end{array}$ & $\begin{array}{c}\text { (Pipe "1) } \\
K \ln , \mathrm{K} \text { Out }\end{array}$ & $\begin{array}{c}\text { (Pipe \#2) } \\
\text { KIn, K Out }\end{array}$ & $\begin{array}{c}\text { (Pipe :3) } \\
\text { KIn, K Out }\end{array}$ & $\begin{array}{l}\text { (Pipe \#4) } \\
\text { KIn, K Out }\end{array}$ & $\begin{array}{c}\text { (Pipe "5) } \\
\text { K In, K Out }\end{array}$ & $\begin{array}{c}\text { (Pipe *6) } \\
K \ln , K \text { Out }\end{array}$ & $\begin{array}{c}\text { (Pipe \#7) } \\
K \ln , K \text { Out }\end{array}$ & $\begin{array}{c}\text { (Pipe *8) } \\
\text { KIn, K Out }\end{array}$ \\
\hline 1 & No & (1) 0,0 & & & & & & & \\
\hline 2 & No & (1) 0,0 & & & & & & & \\
\hline
\end{tabular}

\begin{tabular}{|c|c|c|}
\hline Tank & $\begin{array}{c}\text { (Pipe *9) } \\
\text { KIn, K Out }\end{array}$ & $\begin{array}{c}\text { (Pipe *10) } \\
\text { KIn, K Out }\end{array}$ \\
\hline 1 & & \\
\hline 2 & & \\
\hline
\end{tabular}


Crane Co.

Flow of Fluids Through Valves, Fittings, and Pipe, Technical Paper No. 410, Crane Co., Joliet, IL, 1988

Page 4-13, example 4-20

Sonic choking comparison

Steam flow - Example assumes adiabatic flow

See Verify6.doc MS Word file for comparison with published results

Title: Verify6.aro - Steam flow in a pipe, Example 4-20 from Crane page 4-13

Analysis run on: 12/7/99 11:00:22 AM

Input File: C:AFT Products LAFT ArrowlVerificationiverify6.aro

Execution Times 8.41 seconds

Total Number Of Pressure lterations $=0$

Total Number of Flow lterations= 3

Total Number of Enthalpy Iterations $=3$

Number Of Pipes= 1

Number Of Junctions $=2$

Length March Solution Method with Mach Number Limits

Segments Per Pipe= 10

Mach Number Increment= 01

Preasure Tolerances, 0001 relative change

Mass Flow Rate Tolerancex .0001 relative change

Enthalpy Tolerance $=.0001$ relative change

Flow Relaxation $=.5$

Pressure Relaxation $=.5$

Fluid Database: AFT Standard

Fluid= Steam (Simplified)

Max Fluid Temperature Data 1500 deg. F

Min Fluid Temperature Data $\mathbf{2 0 0}$ deg. F

Molecular Woight $=18.016 \mathrm{amu}$

Gas Constant= 0.110227 Btu/lbm-R

Critical Pressure $=3208.22$ psia

Critical Temperature $=1165.09 \mathrm{deg} . R$

Acentric Factor $=.344$

Equation of State= Redlich-Kwong

Enthalpy Model= Generalized

Specific Heat Ratio Accuracy= High

Atmospheric Pressure= $1 \mathrm{~atm}$

Gravitational Acceleration $=1 \mathrm{~g}$ 's

Standard Pressure= 14.696 psia

Standard Temperature $=60$ deg. $F$

Turbulent Flow Above Reynolds Number $=4000$

Laminar Flow Below Reynolds Number $\mathbf{2 3 0 0}$

Specific Heat Ratio Accuracy= High

Atmospheric Pressure= $1 \mathrm{~atm}$

Gravitational Acceleration $=1 \mathrm{~g}$

Standard Pressure= 14.696 psia

Standard Temperature $=60 \mathrm{deg} . \mathrm{F}$

Turbulent Flow Above Reynolds Number= 4000

Laminar Flow Below Reynolds Number $=\mathbf{2 3 0 0}$

Overall Delta Pressure $=-155.300$ peia

Total Inflow $=3.06717 \mathrm{lbm} / \mathrm{sec}$

Total Outflow= $3.06717 \mathrm{lbm} / \mathrm{sec}$

Total Energy Inflow= 3666.42 Btw/s

Total Energy Outilow= 3666.42 Btuts

Total Heat Transferred Into System $=0.00000 \mathrm{Btu} / \mathrm{s}$

Maximum Pressure is 170.000 paia at Junction 1 Inlet

Minimum Pressure is 14.7000 paia at Junction 2 Outlet

Maximum Static Temperature is 368.480 deg. F at Junction 1 Inlet

Minimum Stetic Temperature is $368.480 \mathrm{deg}$. F at Junction 1 Inlet

Sonic Choking Exists at Junction 2 (Tank)

Pipe Output Table 
SNF-S331, Rov. 0

Verify6.aro - Steam flow in a pipe, Example 4-20 from Crane page 4-13

\begin{tabular}{|c|c|c|c|c|c|c|c|c|c|c|c|c|}
\hline Pipe & $\begin{array}{c}\text { Mass } \\
\text { Flow } \\
\text { (lbm/hr) }\end{array}$ & $\begin{array}{l}\text { Vel. Out } \\
\text { (foet/sec) }\end{array}$ & $\begin{array}{l}\text { Mach } \\
\text { \#Out }\end{array}$ & $\begin{array}{c}\text { P Stag. } \\
\text { In } \\
\text { (pria) }\end{array}$ & $\begin{array}{c}\text { P Stag. } \\
\text { Out } \\
\text { (paia) }\end{array}$ & $\begin{array}{c}\text { P Static } \\
\text { In } \\
\text { (paia) }\end{array}$ & $\begin{array}{c}\text { P Static } \\
\text { Out } \\
\text { (psia) }\end{array}$ & $\begin{array}{c}\text { T Static } \\
\text { In } \\
\text { (deg. F) }\end{array}$ & $\begin{array}{c}\text { T Static } \\
\text { Out } \\
\text { (deg. F) }\end{array}$ & $\begin{array}{c}\text { H Stag. } \\
\text { In } \\
\text { (Btu/bm) }\end{array}$ & $\begin{array}{c}\text { H Stag. } \\
\text { Out } \\
\text { (Btu/hbm) }\end{array}$ & $\begin{array}{c}\text { H Static } \\
\text { In } \\
(B t u / b m)\end{array}$ \\
\hline 1 & $11,041.8$ & 1547.38 & 0.994833 & 170.000 & 63.0569 & 164.638 & 34.9468 & 362.238 & 240.738 & 1195.37 & 1195.37 & 1192.60 \\
\hline
\end{tabular}

\begin{tabular}{|c|c|c|c|c|c|}
\hline Pipe & $\begin{array}{c}\text { H Static } \\
\text { Out } \\
(\text { Btu/bm) }\end{array}$ & $\begin{array}{c}\text { fU } \\
\text { D }+K\end{array}$ & K & fL/D & $f$ \\
\hline 1 & 1147.56 & 11.8597 & 8.53000 & 3.32974 & 0.0191182 \\
\hline
\end{tabular}

All Junction Table

\begin{tabular}{|c|c|c|c|c|c|c|c|c|}
\hline Jet & $\begin{array}{c}\text { P Stag. } \\
\text { In } \\
\text { (psia) }\end{array}$ & $\begin{array}{c}\text { P Stag. } \\
\text { Out } \\
\text { (psia) }\end{array}$ & $\begin{array}{c}\text { P Static } \\
\text { In } \\
\text { (paia) }\end{array}$ & $\begin{array}{c}\text { P Static } \\
\text { Out } \\
\text { (paia) }\end{array}$ & $\begin{array}{c}\text { T Stag. } \\
\text { In } \\
\text { (deg. F) }\end{array}$ & $\begin{array}{c}\text { T Stag. } \\
\text { Out } \\
\text { (deg. F) }\end{array}$ & $\begin{array}{c}\text { T Static } \\
\text { In } \\
\text { (deg. F) }\end{array}$ & $\begin{array}{c}\text { T Static } \\
\text { Out } \\
\text { (deg. F) }\end{array}$ \\
\hline 1 & 170.0000 & 170.0000 & 170.0000 & 170.0000 & 368.480 & 368.480 & 368.480 & 368.480 \\
\hline 2 & 63.0569 & 14.7000 & 63.0569 & 14.7000 & 368.480 & 368.480 & 368.480 & 368.480 \\
\hline
\end{tabular}




\section{TITLE: VERIFY7.ARO}

REFERENCE: Crane Co., Flow of Fluids Through Valves, Fittings, and Pipe, Technical Paper No. 410, Crane Co., Joliet, IL, 1988, Page 4-14, example 4-22

GAS: Air

ASSUMPTIONS: Example does not specify the heat transfer conditions. The AFT Arrow model assumes adiabatic.

RESULTS:

\begin{tabular}{|c|r|r|}
\hline Parameter & Crane & AFT Arrow 2.0 \\
\hline Mass flow rate $(\mathrm{scfm})$ & 62.7 & 63.3 \\
\hline
\end{tabular}

\section{DISCUSSION:}

Crane does not make a distinction between static and stagnation pressure, and it appears that static pressure is usually assumed. From the problem description, the static pressure is clearly appropriate.

The predicted flow rates agree very closely.

The Crane prediction indicates that this pipe will have subsonic velocity at the exit and hence will not choke. However, a more proper formulation of this problem shows that sonic choking will occur. This is a good example of the limitations of simplified methods such as Crane. The discrepancy comes from how to handle the exit loss of the air as it discharges to atmosphere. The Crane solution takes the appropriate $\mathrm{K}$ factor, equal to 1 , and lumps it together with the pipe friction to obtain an overall $\mathrm{K}$ factor of 7.04 .

However, if the $\mathrm{K}$ factor is applied at the discharge tank and not averaged along the pipe, the flow chokes. The predicted choked flow rate is $64.3 \mathrm{scfm}$. This is not drastically different from Crane's prediction, and well within typical engineering uncertainty. But the difference, which is small here, could be larger in other applications.

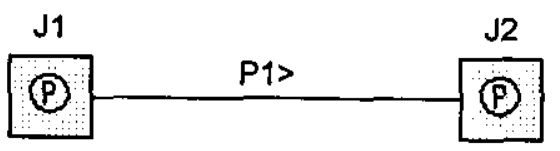

Copyright $\odot 1999$ Applied Flow Technology Corp. All rights reserved. 
SNF-5331, Rev. 0

AFT Arrow 2.0 Input

(1)

12/7/99 11:08 AM

Numatec Hanford Corporation

Verify7.aro - Air flow in a pipe, Example 4-22 from Crane page 4-14

Crane Co.

Flow of Fluids Through Valves, Fittings, and Pipe, Technical Paper No. 410, Crane Co., Joliet, IL, 1988

Page 4-14, example 4-22

Air flow - Example does not specify the heat transfer conditions, so it was assumed adiabatic in this model

See Verify7.doc MS Word file for comparison with published results

Title: Verify7 aro - Air flow in a pipe, Example 4-22 from Crane page 4-14

Number Of Pipes $=1$

Number Of Junctions $=2$

Length March Solution Method with Mach Number Limits

Segments Per Pipe $=10$

Mach Number Increment= .01

Pressure Tolerance $=.0001$ relative change

Mass Flow Rate Tolerance $=.0001$ relative change

Enthalpy Tolerance $=.0001$ relative change

Flow Relaxation $=.5$

Pressure Relaxation $=.5$

Fluid Database: AFT Standard

Fluid $=$ Air

Max Fluid Temperature Data $=1000$ deg. $K$

Min Fluid Temperature Data $=200$ deg. $\mathrm{K}$

Molecular Weight $=28.97 \mathrm{amu}$

Gas Constant $=0.0685486 \mathrm{Btu} / \mathrm{bm}-\mathrm{R}$

Critical Pressure $=37.25 \mathrm{~atm}$

Critical Temperature $=132.41$ deg. $\mathrm{K}$

Acentric Factor $=.021$

Equation of State $=$ Redlich-Kwong

Enthalpy Model= Generalized

Specific Heat Ratio Accuracy= High

Atmospheric Pressure $=1 \mathrm{~atm}$

Gravitational Acceleration $=1 \mathrm{~g}$ 's

Standard Pressure $=14.696 \mathrm{psia}$

Standard Temperature $=60 \mathrm{deg} . F$

Turbulent Flow Above Reynolds Number $=4000$

Laminar Flow Below Reynolds Number $=2300$

Specific Heat Ratio Accuracy $=$ High

Atmospheric Pressure $=1 \mathrm{~atm}$

Gravitational Acceleration $=1 \mathrm{~g}$

Standard Pressure $=14.696$ psia

Standard Temperature $=60$ deg. $F$

Turbulent Flow Above Reynolds Number $=4000$

Laminar Flow Below Reynolds Number $=2300$

Pipe Input Table

\begin{tabular}{|c|r|r|r|r|r|r|r|r|r|r|}
\hline Pipe & Name & $\begin{array}{c}\text { Pipe } \\
\text { Defined }\end{array}$ & Length & $\begin{array}{c}\text { Length } \\
\text { Units }\end{array}$ & $\begin{array}{r}\text { Hydraulic } \\
\text { Diameter }\end{array}$ & $\begin{array}{c}\text { Hydraulic } \\
\text { Diam. Units }\end{array}$ & Roughness & $\begin{array}{c}\text { Roughness } \\
\text { Units }\end{array}$ & Losses (K) & Initial Flow \\
\hline 1 & Pipe & Yes & 10 & feet & 0.546 & inches & 0.00015 & feet & 1 & \\
\hline
\end{tabular}

\begin{tabular}{|c|c|c|c|c|c|c|c|}
\hline Pipe & $\begin{array}{c}\text { Initial Flow } \\
\text { Units }\end{array}$ & $\begin{array}{c}\text { Junctions } \\
(\mathrm{Up}, \text { Down })\end{array}$ & Geometry & Material & Size & Type & $\begin{array}{c}\text { Special } \\
\text { Condition }\end{array}$ \\
\hline 1 & & 1,2 & Cylindrical Pipe & Steel & $1 / 2$ inch & schedule 80 & None \\
\hline
\end{tabular}

\section{Assigned Pressure Table}


SNF-5331, Rev. 0

AFT Arrow 2.0 Input

(2)

12/7/99 11:08 AM

Numatec Hanford Corporation

Verify7.aro - Air flow in a pipe, Example 4-22 from Crane page 4-14

\begin{tabular}{|c|r|r|r|r|r|r|r|r|}
\hline Assigned Pressure & Name & $\begin{array}{c}\text { Object } \\
\text { Defined }\end{array}$ & $\begin{array}{c}\text { Database } \\
\text { Source }\end{array}$ & $\begin{array}{c}\text { Special } \\
\text { Condition }\end{array}$ & Fluid & Pressure & $\begin{array}{c}\text { Pressure } \\
\text { Units }\end{array}$ & Temperature \\
\hline 1 & Assigned Pressure & Yes & & N/A & Air & 19.3 & psig & 100 \\
\hline 2 & Assigned Pressure & Yes & & N/A & Air & 14.7 & psia & 100 \\
\hline
\end{tabular}

\begin{tabular}{|c|r|r|}
\hline Assigned Pressure & $\begin{array}{r}\text { Temperature } \\
\text { Units }\end{array}$ & $\begin{array}{c}\text { Loss } \\
\text { Factor }\end{array}$ \\
\hline 1 & deg. F & 0 \\
\hline 2 & deg. F & 0 \\
\hline
\end{tabular}


Crane Co.

Flow of Fluids Through Valves, Fittings, and Pipe, Technical Paper No. 410, Crane Co., Joliet, IL, 1988

Page 4-14, example 4-22

Air flow - Example does not specify the heat tranofer conditions, 80 lt was assumed adiabatic in this model

See Verify7.doc MS Word file for comparison with published results

Title: Verify7.aro - Air flow in a pipe, Example 4-22 from Crane page 4-14

Analysis run on: 12/7/99 11:07:18 AM

input File: C:LFT ProductsLFT ArrowlVerificationiverify7.aro

Execution Time $=0.88$ seconds

Total Number Of Pressure Iterations $=0$

Total Number Of Flow Iterations $=5$

Total Number Of Enthalpy Iterations= 5

Number Of Pipes= 1

Number Of Junctions $=2$

Length March Solution Method with Mach Number Limits

Segments Per Pipe $=10$

Mach Number Increment= .01

Pressure Tolerance $=0001$ relative change

Mass Flow Rate Tolerance= .0001 relative change

Enthalpy Tolerance $=.0001$ relative change

Flow Relaxation $=.5$

Pressure Relaxation $=.5$

Fluid Database: AFT Standard

Fluid= Air

Max Fluid Temperature Data $=1000$ deg. $K$

Min Fluid Temperature Data $=\mathbf{2 0 0}$ deg. K

Molecular Weight $=28.97 \mathrm{amu}$

Gas Constant $=0.0685486$ Btu/lbm-R

Critical Pressure= $37.25 \mathrm{~atm}$

Critical Temperature $=132.41 \mathrm{deg} . \mathrm{K}$

Acentric Factor= .021

Equation of State= Redlich-Kwong

Enthalpy Model= Generalized

Specific Heat Ratio Accuracy= High

Atmospheric Pressure $=1 \mathrm{~atm}$

Gravitational Acceleration $=1 \mathrm{~g}$ 's

Standard Pressure $=14.696$ psia

Standard Temperature $=60 \mathrm{deg} . F$

Turbulent Flow Above Reynolds Number= 4000

Laminar Flow Below Reynolds Number= 2300

Specific Heat Ratio Accuracy= High

Atmospheric Pressure= $1 \mathrm{~atm}$

Gravitational Acceleration= 1 g

Standard Preasure= 14.696 psia

Standard Temperature $=60$ deg. F

Turbulent Flow Above Reynolds Number= 4000

Laminar Flow Below Reynolds Number= 2300

Overall Delta Pressure $=-17.0789$ psia

Total Inflow $=0.0805723 \mathrm{lbm} / \mathrm{sec}$

Total Outflow= $0.0805723 \mathrm{lbm} / \mathrm{sec}$

Total Energy Inflow= $16.4566 \mathrm{Btu} / \mathrm{s}$

Total Energy Outflow= $16.4566 \mathrm{Btu} / \mathrm{s}$

Total Heat Transferred Into System= $0.00000 \mathrm{Btu} / \mathrm{s}$

Maximum Pressure is 33.9960 poia at Junction 1 Inlet

Minimum Pressure is 14.6994 paia at Junction 2 Inlet

Maximum Static Temperature is $137.944 \mathrm{deg}$. F at Junction 2 Inlet

Minimum Static Temperature is $107.466 \mathrm{deg}$. F at Junction 1 Inlet

\section{Pipe Output Table}


Verify7.aro - Air flow in a pipe, Example 4-22 from Crane page 4-14

\begin{tabular}{|c|c|c|c|c|c|c|c|c|c|c|c|c|}
\hline Pipe & $\begin{array}{c}\text { Mass } \\
\text { Flow } \\
\text { (acfm) }\end{array}$ & $\begin{array}{c}\text { Vel. In } \\
\text { (feet/sec) }\end{array}$ & $\begin{array}{l}\text { Vel. Out } \\
\text { (feetsec) }\end{array}$ & $\begin{array}{c}\text { Mach } \\
\text { * in }\end{array}$ & $\begin{array}{l}\text { Mach } \\
* \text { Out }\end{array}$ & $\begin{array}{c}\text { P Static } \\
\text { In } \\
\text { (paia) }\end{array}$ & $\begin{array}{c}\text { P Static } \\
\text { Out } \\
\text { (pria) }\end{array}$ & $\begin{array}{c}\text { dP Static } \\
\text { Total } \\
\text { (psid) }\end{array}$ & $\begin{array}{c}\text { T Static } \\
\text { In } \\
\text { (deg. F) }\end{array}$ & $\begin{array}{c}\text { T Static } \\
\text { Out } \\
\text { (deg. F) }\end{array}$ & K & fLD \\
\hline 1 & 63.2892 & 301.942 & 662.431 & 0.261373 & 0.588539 & 33.9960 & 14.6995 & 19.2965 & 100.000 & 71.0659 & 1.00000 & 6.09426 \\
\hline
\end{tabular}

\begin{tabular}{|c|c|c|}
\hline Pipe & $\begin{array}{c}\text { fU } \\
D+K\end{array}$ & \\
\hline 1 & 7.09426 & 0.0277289 \\
\hline
\end{tabular}

All Junction Table

\begin{tabular}{|c|c|c|c|c|}
\hline Jct & $\begin{array}{c}\text { P Static } \\
\text { In } \\
\text { (psia) }\end{array}$ & $\begin{array}{c}\text { P Static } \\
\text { Out } \\
\text { (psia) }\end{array}$ & $\begin{array}{c}\text { T Static } \\
\text { In } \\
\text { (deq. F) }\end{array}$ & $\begin{array}{c}\text { T Static } \\
\text { Out } \\
\text { (deg. F) }\end{array}$ \\
\hline 1 & 33.9960 & 33.9960 & 100.0000 & 100.0000 \\
\hline 2 & 14.6994 & 14.6994 & 99.9938 & 99.9938 \\
\hline
\end{tabular}




\section{TITLE: VERIFY8.ARO}

REFERENCE: Robert W. Fox and Alan T. McDonald, Introduction to Fluid Mechanics, Third Edition, John Wiley \& Sons, 1985, Pages 632-633, example 12.8

GAS: Air

ASSUMPTIONS: 1) Adiabatic flow, 2) Perfect gas.

RESULTS:

\begin{tabular}{|l|r|r|}
\hline \multicolumn{1}{|c|}{ Parameter } & $\begin{array}{c}\text { Fox \& } \\
\text { McDonald }\end{array}$ & AFT Arrow 2.0 \\
\hline$L_{1-3}$ - length to choking (meters) & 4.99 & 4.994 \\
\hline$V_{1}-$ Velocity at point 1 (m/s) & 65.3 & 65.2 \\
\hline$T_{1}-$ Temperature at point 1 (deg, K) & 294 & 293.9 \\
\hline$M_{1}-$ Mach number at point 1 & 0.19 & 0.19 \\
\hline$M_{2}-$ Mach number at point 2 & 0.40 & 0.40 \\
\hline$P_{2}-$ Pressure at point 2 (mm Hg gage) & -18.9 & $-18.86^{*}$ \\
\hline $\begin{array}{l}L_{1-2}-\text { length to measured pressure } \\
\text { (meters) }\end{array}$ & 4.29 & $4.29^{*}$ \\
\hline
\end{tabular}

* AFT Arrow does not have the ability to solve for pipe length, so the length was input. With this known length, the AFT Arrow Mach number at $M_{2}$ should agree with Fox \& McDonald's, and it does. The resulting mass flow rate is then used as input for AFT Arrow pipe \#2.

\section{DISCUSSION:}

The predictions agree very closely.

The two pipes in the AFT Arrow model represent the solutions to stations 2 and 3.

Note that the friction factor in Fox \& McDonald is the Fanning friction factor. To obtain the Darcy-

Weisbach friction factor used in AFT Arrow, multiply the Fanning friction factor by 4.

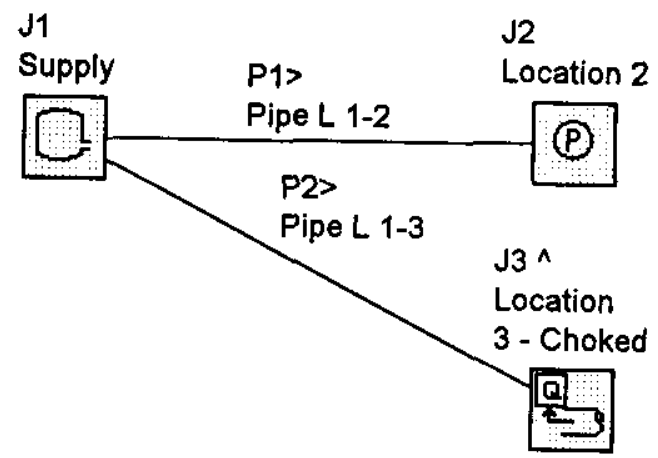

Copyright $(\mathcal{O} 1999$ Applied Flow Technology Corp. All rights reserved. 
SNF-5331, Rev. 0

AFT Arrow 2.0 Input

12//99 11:13 AM

Numatec Hanford Corporation

Verify8.aro - Air flow in a pipe, Example 12.8 from Fox \& McDonald page 632-3

Robert W. Fox and Alan T. McDonald

Introduction to Fluid Mechanics, Third Edition, John Wiley \& Sons, 1985

Pages 632-633, example 12.8

Air flow - The two pipes represent the solutions to stations 2 and 3.

See Verify8.doc MS Word file for comparison with published results

Title: Verify8.aro - Air flow in a pipe, Example 12.8 from Fox \& McDonald page 632-3

Number Of Pipes $=2$

Number Of Junctions $=3$

Length March Solution Method with Mach Number Limits

Segments Per Pipe= 10

Mach Number Increment $=.01$

Pressure Tolerance $=.0001$ relative change

Mass Flow Rate Tolerance $=.0001$ relative change

Enthalpy Tolerance $=.0001$ relative change

Concentration Tolerance $=.0001$ relative change

Flow Relaxation $=.5$

Pressure Relaxation $=.5$

Fluid Database: AFT Standard

Fluid= Air

Max Fluid Temperature Data $=1000$ deg. $\mathrm{K}$

Min Fluid Temperature Data $=200$ deg. $K$

Molecular Weight $=28.97 \mathrm{amu}$

Gas Constant $=0.06855 \mathrm{Btu} / \mathrm{bm}-\mathrm{R}$

Critical Pressure $=37.25$ atm

Critical Temperature $=132.41 \mathrm{deg} . \mathrm{K}$

Acentric Factor $=.021$

Equation of State= Ideal Gas

Enthalpy Model= Reference

Specific Heat Ratio Accuracy $=$ High

Atmospheric Pressure= $1 \mathrm{~atm}$

Gravitational Acceleration $=1 \mathrm{~g}$ 's

Standard Pressure= 14.696 psia

Standard Temperature $=60$ deg. $F$

Turbulent Flow Above Reynolds Number $=4000$

Laminar Flow Below Reynolds Number $=2300$

Specific Heat Ratio Accuracy $=$ High

Atmospheric Pressure $=1 \mathrm{~atm}$

Gravitational Acceleration $=1 \mathrm{~g}$

Standard Pressure $=14.696$ psia

Standard Temperature $=60 \mathrm{deg} . \mathrm{F}$

Turbulent Flow Above Reynolds Number $=4000$

Laminar Flow Below Reynolds Number $=2300$

\section{Pipe Input Table}

\begin{tabular}{|c|c|r|r|r|r|r|r|r|r|r|}
\hline Pipe & Name & $\begin{array}{c}\text { Pipe } \\
\text { Defined }\end{array}$ & Length & $\begin{array}{c}\text { Length } \\
\text { Units }\end{array}$ & $\begin{array}{r}\text { Hydraulic } \\
\text { Diameter }\end{array}$ & $\begin{array}{c}\text { Hydraulic } \\
\text { Diam. Units }\end{array}$ & Roughness & $\begin{array}{c}\text { Roughness } \\
\text { Units }\end{array}$ & Losses (K) & Initial Flow \\
\hline 1 & Pipe L 1-2 & Yes & 4.29 & meters & 7.16 & $\mathrm{~mm}$ & 0.0235 & Explicit $\mathrm{f}$ & 0 & \\
\hline 2 & Pipe L 1-3 & Yes & 4.994 & meters & 7.16 & $\mathrm{~mm}$ & 0.0235 & Explicit f & \\
\hline
\end{tabular}

\begin{tabular}{|c|c|r|c|c|c|c|c|}
\hline Pipe & $\begin{array}{c}\text { Initial Flow } \\
\text { Units }\end{array}$ & $\begin{array}{r}\text { Junctions } \\
\text { (Up,Down) }\end{array}$ & Geometry & Material & Size & Type & $\begin{array}{c}\text { Special } \\
\text { Condition }\end{array}$ \\
\hline 1 & & 1,2 & Cylindrical Pipe & Unspecified & & & None \\
\hline 2 & & 1,3 & Cylindrical Pipe & Unspecified & & & None \\
\hline
\end{tabular}


SNF-5331, Rev. 0

AFT Arrow 2.0 Input

Numatec Hanford Corporation
(2)

$12 \pi / 9911: 13 \mathrm{AM}$

Verify8.aro - Air flow in a pipe, Example 12.8 from Fox \& McDonald page 632-3

\section{Assigned Flow Table}

\begin{tabular}{|c|c|r|r|r|r|r|r|r|r|}
\hline Assigned Flow & Name & $\begin{array}{c}\text { Object } \\
\text { Defined }\end{array}$ & $\begin{array}{c}\text { Database } \\
\text { Source }\end{array}$ & $\begin{array}{c}\text { Special } \\
\text { Condition }\end{array}$ & $\begin{array}{r}\text { Fluid } \\
\text { Colow }\end{array}$ & Type & Flow & $\begin{array}{c}\text { Flow } \\
\text { Units }\end{array}$ & $\begin{array}{c}\text { Temperature } \\
\text { - }\end{array}$ \\
\hline 3 & Location 3 - Choked & Yes & & None & Air & Outflow & $3.07637 \mathrm{E}-03$ & $\mathrm{~kg} / \mathrm{sec}$ & 286 \\
\hline
\end{tabular}

\begin{tabular}{|c|c|r|}
\hline Assigned Flow & $\begin{array}{c}\text { Temperature } \\
\text { Units }\end{array}$ & $\begin{array}{c}\text { Loss } \\
\text { Model }\end{array}$ \\
\hline 3 & deg. K & 0 \\
\hline
\end{tabular}

\section{Assioned Pressure Table}

\begin{tabular}{|c|c|c|c|c|c|c|c|c|c|}
\hline Assigned Pressure & Name & $\begin{array}{c}\text { Object } \\
\text { Defined }\end{array}$ & $\begin{array}{c}\text { Database } \\
\text { Source }\end{array}$ & $\begin{array}{c}\text { Special } \\
\text { Condition }\end{array}$ & Fluid & $\begin{array}{c}\text { Pressure } \\
\text { Pressure } \\
\text { Units }\end{array}$ & $\begin{array}{c}\text { Temperature } \\
\text { Temperature } \\
\text { Units }\end{array}$ \\
\hline 2 & Location 2 & Yes & & N/A & Air & -412 & $\mathrm{~mm} \mathrm{Hg}(\mathrm{g})$ & 296 & deg. K \\
\hline
\end{tabular}

\begin{tabular}{|c|r|}
\hline Assigned Pressure & $\begin{array}{c}\text { Loss } \\
\text { Factor }\end{array}$ \\
\hline 2 & 0 \\
\hline
\end{tabular}

\section{Tank Table}

\begin{tabular}{|c|c|c|c|r|r|r|r|r|r|r|}
\hline Tank & Name & $\begin{array}{c}\text { Object } \\
\text { Defined }\end{array}$ & $\begin{array}{c}\text { Database } \\
\text { Source }\end{array}$ & $\begin{array}{c}\text { Special } \\
\text { Condition }\end{array}$ & Fluid & Pressure & $\begin{array}{c}\text { Pressure } \\
\text { Units }\end{array}$ & Temperature & $\begin{array}{c}\text { Temperature } \\
\text { Units }\end{array}$ & $\begin{array}{c}\text { Balance } \\
\text { Energy }\end{array}$ \\
\hline 1 & Supply & Yes & & N/A & Air & 760 & $\mathrm{~mm} \mathrm{Hg}$ & 296 & deg. K & No \\
\hline
\end{tabular}

\begin{tabular}{|c|c|c|c|c|c|c|c|c|c|}
\hline Tank & $\begin{array}{c}\text { Balance } \\
\text { Concentration }\end{array}$ & $\begin{array}{c}\text { (Pipe \#1) } \\
\text { K In, K Out }\end{array}$ & $\begin{array}{c}\text { (Pipe \#2) } \\
\text { KIn, K Out }\end{array}$ & $\begin{array}{c}\text { (Pipe \#3) } \\
\text { KIn, K Out }\end{array}$ & $\begin{array}{c}\text { (Pipe \#4) } \\
\text { KIn, K Out }\end{array}$ & $\begin{array}{c}\text { (Pipe \#5) } \\
\text { KIn, K Out }\end{array}$ & $\begin{array}{c}\text { (Pipe \#6) } \\
\text { K In, K Out }\end{array}$ & $\begin{array}{c}\text { (Pipe \#7) } \\
\text { K In, K Out }\end{array}$ & $\begin{array}{c}\text { (Pipe \#8) } \\
\text { K In, K Out }\end{array}$ \\
\hline 1 & No & (1) 0,0 & (2) 0,0 & & & & & & \\
\hline
\end{tabular}

\begin{tabular}{|c|c|c|}
\hline Tank & $\begin{array}{c}\text { (Pipe *9) } \\
\text { K In, K Out }\end{array}$ & $\begin{array}{c}\text { (Pipe *10) } \\
\text { K In, K Out }\end{array}$ \\
\hline 1 & & \\
\hline
\end{tabular}


Robert W. Fox and Alan T. McDonald

Introduction to Fluid Mechanics, Third Edition, John Wiley \& Sons, 1985

Pages 632-633, example 12.8

Air flow - The two pipes represent the solutions to stations 2 and 3 .

See Verify8.doc MS Word file for comparison with published results

Title: Verify8.aro - Air flow in a pipe, Example 12.8 from Fox \& McDonald page 632-3

Analysis run on: 12/799 11:13:38 AM

Input File: C:LAFT ProductsLFT ArrowlVerificationiverify8.aro

Execution Time $=1.10$ seconds

Total Number Of Pressure Iterations $=0$

Total Number Of Flow iterations $=5$

Total Number Of Enthalpy Iterations $=5$

Number Of Pipes $=2$

Number Of Junctions $=3$

Length March Solution Method with Mach Number Limits

Segments Per Pipe $=10$

Mach Number Increment $=.01$

Pressure Tolerance $=.0001$ relative change

Mass Flow Rate Tolerance $=.0001$ relative change

Enthalpy Tolerance $=.0001$ relative change

Flow Relaxation $=.5$

Pressure Relaxation $=.5$

Fluid Database: AFT Standard

Fluid= Air

Max Fluid Temperature Data $=1000$ deg. $K$

Min Fluid Temperature Data $=200$ deg. K

Molecular Weight $=28.97 \mathrm{amu}$

Gas Constant $=0.06855$ Btu/bm-R

Critical Pressure $=37.25 \mathrm{~atm}$

Critical Temperature $=132.41 \mathrm{deg} . \mathrm{K}$

Acentric Factor $=.021$

Equation of State $=$ Ideal Gas

Enthalpy Model= Reference

Specific Heat Ratio Accuracy= High

Atmospheric Pressure $=1 \mathrm{~atm}$

Gravitational Acceleration= $1 \mathrm{~g}$ 's

Standard Pressure $=14.696$ psia

Standard Temperature $=60 \mathrm{deg}$. F

Turbulent Flow Above Reynolds Number $=4000$

Laminar Flow Below Reynolds Number $=2300$

Specific Heat Ratio Accuracy $=$ High

Atmospheric Pressure $=1 \mathrm{~atm}$

Gravitational Acceleration $=1 \mathrm{~g}$

Standard Pressure $=14.696$ psia

Standard Temperature $=60 \mathrm{deg} . F$

Turbulent Flow Above Reynolds Number $=4000$

Laminar Flow Below Reynolds Number $=2300$

Total Inflow $=0.01356 \mathrm{lbm} / \mathrm{sec}$

Total Outflow $=0.01356 \mathrm{lbm} / \mathrm{sec}$

Total Energy Inflow= 2.660 Btu/s

Total Energy Outflow $=2.660 \mathrm{Btu} / \mathrm{s}$

Total Heat Transferred Into System $=0.000 \mathrm{Btu} / \mathrm{s}$

Maximum Pressure is 14.70 psia at Junction 1 inlet

Minimum Pressure is 2.551 psia at Junction 3 Inlet

Maximum Static Temperature is 89.90 deg. $F$ at Junction 2 Inlet

Minimum Static Temperature is $73.13 \mathrm{deg}$. F at Junction 1 inlet

\section{Pipe Output Table}




\begin{tabular}{|c|c|c|c|c|c|c|c|c|c|c|c|}
\hline Pipe & Name & $\begin{array}{l}\text { Length } \\
\text { (moters) }\end{array}$ & $\begin{array}{c}\text { Mass } \\
\text { Flow } \\
(\mathrm{kg} / \mathrm{sec})\end{array}$ & $\begin{array}{c}\text { Vel. } \\
\text { In } \\
\text { (meters/sec) }\end{array}$ & $\begin{array}{c}\text { Vol. } \\
\text { Out } \\
\text { (motera/sec) }\end{array}$ & $\begin{array}{c}\text { Mach } \\
\# \text { in }\end{array}$ & $\begin{array}{l}\text { Mach } \\
\text { \# Out }\end{array}$ & $\begin{array}{c}\text { P Stag. } \\
\text { In } \\
(\mathrm{mm} \mathrm{Hg})\end{array}$ & $\begin{array}{c}\text { P Static } \\
\text { In } \\
(\mathrm{mm} \mathrm{Hg}(g))\end{array}$ & $\begin{array}{c}\text { P Static } \\
\text { Out } \\
(\mathrm{mm} \mathrm{Hg}(\mathrm{g}))\end{array}$ & $\begin{array}{c}\text { T Stag. } \\
\text { In } \\
\text { (deg. } \mathrm{K} \text { ) }\end{array}$ \\
\hline 1 & Pipe L 1-2 & 4.290 & $3.076 E-03$ & 65.23 & 135.6 & 0.1903 & 0.4005 & 760.0 & -18.86 & -412.0 & 296.0 \\
\hline 2 & Pipe L 1-3 & 4.994 & $3.076 E-03$ & 65.23 & 310.1 & 0.1903 & 0.9829 & 760.0 & -18.86 & -628.1 & 296.0 \\
\hline
\end{tabular}

\begin{tabular}{|c|r|r|r|r|r|}
\hline Pipe & $\begin{array}{c}\text { T Stag. } \\
\text { Out } \\
\text { (deg. K) }\end{array}$ & $\begin{array}{c}\text { T Static } \\
\text { In } \\
\text { (deg. K) }\end{array}$ & $\begin{array}{c}\text { T Static } \\
\text { Out } \\
\text { (deq. K) }\end{array}$ & $\begin{array}{c}\text { f } \\
\begin{array}{c}\text { Rho } \\
\text { Static In } \\
\text { (ko/m3) }\end{array}\end{array}$ \\
\hline 1 & 296.0 & 293.9 & 287.0 & 0.02350 & 1.171 \\
\hline 2 & 296.1 & 293.9 & 248.7 & 0.02350 & 1.171 \\
\hline
\end{tabular}

All Junction Table

\begin{tabular}{|c|c|c|c|c|c|c|c|c|c|}
\hline Jet & Name & $\begin{array}{c}\text { P Stag. } \\
\text { In } \\
(\mathrm{mm} \mathrm{Ho})\end{array}$ & $\begin{array}{l}\text { P Stag. } \\
\text { Out } \\
(\mathrm{mm} \mathrm{Hg})\end{array}$ & $\begin{array}{c}\text { P Static } \\
\text { In } \\
(\mathrm{mm} \mathrm{Ho})\end{array}$ & $\begin{array}{c}\text { P Static } \\
\text { Out } \\
(\mathrm{mm} \mathrm{Hg})\end{array}$ & $\begin{array}{c}\text { T Stag. } \\
\text { In } \\
\text { (deg. K) }\end{array}$ & $\begin{array}{l}\text { TStag. } \\
\text { Out } \\
\text { (dog. K) }\end{array}$ & $\begin{array}{c}\text { T Static } \\
\text { In } \\
(\operatorname{deg} . K)\end{array}$ & $\begin{array}{c}\text { T Static } \\
\text { Out } \\
\text { (deg. K) }\end{array}$ \\
\hline 1 & Supply & 760.0 & 760.0 & 760.0 & 760.0 & 296.0 & 296.0 & 296.0 & 296.0 \\
\hline 2 & Location 2 & 388.4 & 388.4 & 348.0 & 348.0 & 305.3 & 305.3 & 296.0 & 296.0 \\
\hline 3 & 3- Choked & 244.4 & 244.4 & 131.9 & 131.9 & 296.1 & 296.1 & 248.7 & 248.7 \\
\hline
\end{tabular}


TITLE: VERIFY9.ARO

REFERENCE: Michael R. Lindeburg, P.E., Mechanical Engineering Review Manual, Seventh Edition, Professional Publications, Belmont, CA, 1984, Pages 8-11, 8-12, example 8.12

GAS: Unspecified except that the $k$ value (i.e., $\gamma$ ) is 1.4

ASSUMPTIONS: 1) Adiabatic flow, 2) Perfect gas, 3) The gas is air, but for purposes of the example it only matters that the gas has $k=1.4,4$ ) No temperature was specified, so assume $70 \mathrm{deg}$. F

RESULTS:

\begin{tabular}{|c|c|c|}
\hline Parameter & Lindeburg & AFT Arrow 2.0 \\
\hline$M_{2}-$ Mach number at exit & 0.35 & 0.356 \\
\hline$P_{2}-$ Pressure at exit (psia) & 10.26 & 10.09 \\
\hline
\end{tabular}

\section{DISCUSSION:}

As specified, inlet conditions are known and outlet conditions need to be determined. With the known inlet conditions, an implied mass flow rate exists. To pose the problem in AFT Arrow terms, a few simple calculations are needed to obtain the mass flow rate. Once obtained, it is applied as a flow demand at the exit.

The problem states that the inlet Mach number is $0.3, P_{1}=12 \mathrm{psia}, T_{1}=70 \mathrm{~F}$ (assumed). From the ideal gas law, density, sonic speed and mass flow rate are:

$$
\begin{aligned}
& \rho_{1}=\frac{P_{1}}{R T_{1}}=\frac{(12 \mathrm{psia})\left(144 \mathrm{in}^{2} / \mathrm{ft}^{2}\right)}{\left(53.34 \frac{\mathrm{ft} \cdot \mathrm{lbf}}{\mathrm{lbm} \cdot \mathrm{R}}\right)(529.67 \mathrm{R})}=0.06116 \mathrm{lbm} / \mathrm{ft}^{3} \\
& a_{1}=\sqrt{\gamma R T_{1}}=\sqrt{1.4\left(53.34 \frac{\mathrm{ft} \cdot \mathrm{lbf}}{\mathrm{lbm} \cdot \mathrm{R}}\right) 529.67 \mathrm{R}}=1128.1 \mathrm{ft} / \mathrm{s} \text { (sonic velocity) } \\
& \dot{m}=\rho_{1} V_{1} A=\rho_{1}\left(M_{1} a_{1}\right) A=\left(0.6116 \frac{\mathrm{lbm}}{\mathrm{ft}^{3}}\right)(0.3)\left(1128.1 \frac{\mathrm{ft}}{\mathrm{s}}\right)\left(\frac{\pi}{4} 0.3^{2} \mathrm{ft}^{2}\right)=1.463 \mathrm{lbm} / \mathrm{s}
\end{aligned}
$$

With this flow rate at the exit, the predictions agree very closely.

Note that the friction factor in Lindeburg is the Fanning friction factor. To obtain the Darcy-Weisbach friction factor used in AFT Arrow, multiply the Fanning friction factor by 4 .

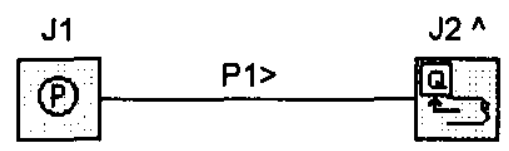

Copyright 1999 Applied Flow Technology Corp. All rights reserved. 
Michael R. Lindeburg, P.E.

Mechanical Engineering Review Manual, Seventh Edition, Profeasional Publications, Belmont, CA, 1984

Pages 8-11, 8-12, example 8.12

Gas flow - Assumed to be air for this model, but for purposes of the example it only matters that the gas has $k=1.4$

See Verify9.doc MS Word file for comparison with publiahed results

Title: Verify9.aro - Air flow in a pipe, Example 8.12 from Lindeburg page 8-11

Number Of Pipes $=1$

Number Of Junctions= 2

Length March Solution Method with Mach Number Limits

Segments Per Pipes 10

Mach Number Increment= 01

Pressure Tolerance $=.0001$ relative change

Mass Flow Rate Tolerance= .0001 relative change

Enthalpy Tolerances .0001 relative change

Concentration Tolerancex .0001 relative change

Flow Relaxation $=.5$

Pressure Relaxation $=.5$

Fluid Database: AFT Standard

Fluid= Air

Max Fluid Temperature Data 1000 deg. $K$

Min Fluid Temperature Data $=200$ deg. K

Molecular Weight= $28.97 \mathrm{amu}$

Gas Constant= 53.3424 ft-lbf/hbm-R

Critical Pressure $=37.25 \mathrm{~atm}$

Critical Temperature= $132.41 \mathrm{deg} . \mathrm{K}$

Acentric Factor $=.021$

Equation of Statex Ideal Gas

Enthalpy Model= Reference

Specific Heat Ratio Accuracy= High

Atmospheric Pressure= $1 \mathrm{~atm}$

Gravitational Acceleration $=1 \mathrm{~g}$ 's

Standard Pressure= 14.696 paia

Standard Temperature $=60 \mathrm{deg}$. $F$

Turbulent Flow Above Reynolds Number= 4000

Laminar Flow Below Reynolds Number= 2300

Specific Heat Ratio Accuracy= High

Atmospheric Pressure $=1 \mathrm{~atm}$

Gravitational Acceleration $=1$ s

Standard Preasure= 14.696 psia

Standard Temperature= $60 \mathrm{deg}$. F

Turbulent Flow Above Reynolds Number $=4000$

Laminar Flow Below Reynolds Number= 2300

Pipe Input Table

\begin{tabular}{|c|r|r|r|r|r|r|r|r|r|r|}
\hline Pipe & Name & $\begin{array}{c}\text { Pipe } \\
\text { Defined }\end{array}$ & Length & $\begin{array}{r}\text { Length } \\
\text { Units }\end{array}$ & $\begin{array}{c}\text { Hydraullic } \\
\text { Diameter }\end{array}$ & $\begin{array}{c}\text { Hydraulic } \\
\text { Diam. Units }\end{array}$ & Roughness & $\begin{array}{c}\text { Roughness } \\
\text { Units }\end{array}$ & Losses (K) & Initial Flow \\
\hline 1 & Pipe & Yes & 50 & feet & 0.3 & foet & 0.012 & Explicit f & 0 & \\
\hline
\end{tabular}

\begin{tabular}{|c|r|r|c|c|c|c|c|}
\hline Pipe & $\begin{array}{c}\text { Initial Flow } \\
\text { Units }\end{array}$ & $\begin{array}{c}\text { Junctions } \\
(\text { Up,Down) }\end{array}$ & Geometry & Material & Size & Type & $\begin{array}{c}\text { Special } \\
\text { Condition }\end{array}$ \\
\hline 1 & & 1,2 & Cylindrical Pipe & Unapecified & & & None \\
\hline
\end{tabular}

\section{Assjoned Flow Table}


SNF-5331, Rev. 0

AFT Arrow 2.0 Input

(2)

12/7/99 11:16 AM

Numatec Hanford Corporation

Verify9.aro - Air flow in a pipe, Example 8.12 from Lindeburg page 8-11

\begin{tabular}{|c|c|r|r|r|r|r|r|r|r|}
\hline Assigned Flow & Name & $\begin{array}{c}\text { Object } \\
\text { Defined }\end{array}$ & $\begin{array}{c}\text { Database } \\
\text { Source }\end{array}$ & $\begin{array}{c}\text { Special } \\
\text { Condition }\end{array}$ & Fluid & Type & Flow & $\begin{array}{c}\text { Flow } \\
\text { Units }\end{array}$ & Temperature \\
\hline 2 & Assigned Flow & Yes & & None & Air & Outflow & 1.4626 & lbm/sec & 70 \\
\hline
\end{tabular}

\begin{tabular}{|c|c|c|}
\hline Assigned Flow & $\begin{array}{c}\text { Temperature } \\
\text { Units }\end{array}$ & $\begin{array}{c}\text { Loss } \\
\text { Model }\end{array}$ \\
\hline 2 & deg. F & 0 \\
\hline
\end{tabular}

\section{Assigned Pressure Table}

\begin{tabular}{|c|c|r|r|r|r|r|r|r|}
\hline Assigned Pressure & Name & $\begin{array}{c}\text { Object } \\
\text { Defined }\end{array}$ & $\begin{array}{c}\text { Database } \\
\text { Source }\end{array}$ & $\begin{array}{c}\text { Special } \\
\text { Condition }\end{array}$ & Fluid & Pressure & $\begin{array}{c}\text { Pressure } \\
\text { Units }\end{array}$ & \begin{tabular}{c} 
Temperature \\
\hline 1
\end{tabular} \\
\hline 1 & Assigned Pressure & Yes & & N/A & Air & 12 & psia & 70 \\
\hline
\end{tabular}

\begin{tabular}{|c|r|r|}
\hline Assigned Pressure & $\begin{array}{c}\text { Temperature } \\
\text { Units }\end{array}$ & $\begin{array}{c}\text { Loss } \\
\text { Factor }\end{array}$ \\
\hline 1 & deg. F & 0 \\
\hline
\end{tabular}


Michael R. Lindeburg, P.E.

Mechanical Engineering Review Manual, Seventh Edition, Profeesional Publications, Belmont, CA, 1984

Pages 8-11, 8-12, example 8.12

Gas flow - Assumed to be air for this model, but for purposes of the example it only matters that the gas has $k=1.4$

See Verify9.doc MS Word file for comparison with published results

Title: Verify9.aro - Air flow in a pipe, Example 8.12 from Lindeburg page 8-11

Analysis run on: 12/799 11:21:15 AM

Input File: C:VAFT ProductsLAFT ATrowlVerificationlverify9.aro

Execution Time $=0.22$ seconds

Total Number Of Pressure Iterations $=0$

Total Number Of Flow lterations= 2

Total Number Of Enthalpy lterations= 2

Number Of Pipes= 1

Number Of Junctions= 2

Length March Solution Method with Mach Number Limita

Segments Per Pipe $=10$

Mach Number Increment= 01

Preasure Tolerance= .0001 relative change

Mass Flow Rate Tolerance .0001 relative change

Enthalpy Tolerancer .0001 relative change

Flow Relaxation $=.5$

Pressure Relaxation $=.5$

Fluid Database: AFT Standard

Fluid= Air

Max Fluid Temperature Data $=1000$ deg. $K$

Min Fluid Temperature Data $=200$ deg. $K$

Molecular Weightw 28.97 amu

Gas Constant= 53.3424 ft-lbf/lbm-R

Critical Pressure $=37.25$ atm

Critical Temperature= 132.41 deg. $K$

Acentric Factor= .021

Equation of State= Ideal Gas

Enthalpy Model= Reference

Specific Heat Ratio Accuracy= High

Atmospheric Pressure $=1 \mathrm{~atm}$

Gravitational Acceleration $=1 \mathrm{~g} / \mathrm{s}$

Standard Pressure= 14.696 psia

Standard Temperature $=60 \mathrm{deg} . F$

Turbulent Flow Above Reynolds Number= 4000

Laminar Flow Below Reynolds Number $=\mathbf{2 3 0 0}$

Specific Heat Ratio Accuracy= High

Atmospheric Pressure= 1 atm

Gravitational Acceleration $=1 \mathrm{~g}$

Standard Preasure= 14.696 psia

Standard Temperature $=60 \mathrm{deg}$. F

Turbulent Flow Above Reynolds Numbers $\mathbf{4 0 0 0}$

Laminar Flow Below Reynolds Number $=\mathbf{2 3 0 0}$

Overall Delta Pressure $=-1.76057$ psia

Total Inflow= $1.46260 \mathrm{lbm} / \mathrm{sec}$

Total Outflow= $1.46260 \mathrm{lbm} / \mathrm{sec}$

Total Energy Inflow= $289.019 \mathrm{Btw} / \mathrm{s}$

Total Energy Outllow $=289.019 \mathrm{Btw} / \mathrm{s}$

Total Heat Transferred into System $=0.00000 \mathrm{Btu} / \mathrm{s}$

Maximum Pressure is 12.0000 psia at Junction 1 Inlet

Minimum Pressure is 10.0914 peia at Junction 2 Inlet

Maximum Static Temperature is 79.3964 deg. F at Junction 2 Inlet

Minimum Static Temperature is $79.3949 \mathrm{deg}$. F at Junction 1 Inlet

\section{Pipe Output Table}


Verify9.aro - Air flow in a pipe, Example 8.12 from Lindeburg page 8-11

\begin{tabular}{|c|c|c|c|c|c|c|c|}
\hline Pipe & $\begin{array}{c}\text { Mach } \\
\text { In }\end{array}$ & $\begin{array}{c}\text { Mach } \\
\text { \# Out }\end{array}$ & $\begin{array}{c}\text { Mass } \\
\text { Flow } \\
(\text { bm/sec) }\end{array}$ & $\begin{array}{c}\text { P Static } \\
\text { In } \\
\text { (poia) }\end{array}$ & $\begin{array}{c}\text { P Static } \\
\text { Out } \\
\text { (psia) }\end{array}$ & $\begin{array}{c}\text { Vel. } \\
\text { Sonic In } \\
\text { (fect/sec) }\end{array}$ & $\begin{array}{c}\text { Rho } \\
\text { Static In } \\
(\text { (bm/n3) }\end{array}$ \\
\hline 1 & 0.300730 & 0.356346 & 1.46260 & 12.0000 & 10.0914 & 1125.00 & 0.0611597 \\
\hline
\end{tabular}

\section{All Junction Table}

\begin{tabular}{|c|c|c|}
\hline Jet & $\begin{array}{c}\text { P Static } \\
\text { In } \\
\text { (psia) }\end{array}$ & $\begin{array}{c}\text { P Static } \\
\text { Out } \\
\text { (pgia) }\end{array}$ \\
\hline 1 & 12.0000 & 12.0000 \\
\hline 2 & 10.0914 & 10.0914 \\
\hline
\end{tabular}


TITLE: VERIFY 10.ARO

REFERENCE: Michael R. Lindeburg, P.E., Mechanical Engineering Review Manual, Seventh Edition, Professional Publications, Belmont, CA, 1984, Pages 8-12, 8-13, example 8.14.

GAS: Methane

ASSUMPTIONS: 1) Isothermal flow, 2) Perfect gas

RESULTS:

\begin{tabular}{|c|c|c|}
\hline Parameter & Lindebourg & AFT Arrow 2.0 \\
\hline Mass flow rate $(\mathrm{lbm} / \mathrm{s})$ & 456 & 478 \\
\hline
\end{tabular}

\section{DISCUSSION:}

The predictions agree closely. Part "a" makes a comparison to the Bernoulli equation, which Arrow does not solve. So part "a" was skipped.

Note that the friction factor in Lindeburg is the Fanning friction factor. To obtain the Darcy-Weisbach friction factor used in AFT Arrow, multiply the Fanning friction factor by 4.

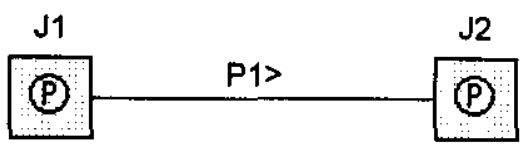

Copyright (C) 1999 Applied Flow Technology Corp. All rights reserved. 
SNF-5331, Rev. 0

AFT Arrow 2.0 Input

Numatec Hanford Corporation

Verify10.aro - Methane flow in a pipe, Example 8.13b from Lindeburg page 8-12

Michael R. Lindeburg, P.E.

Mechanical Engineering Review Manual, Seventh Edition, Professional Publications, Belmont, CA, 1984

Pages 8-12, 8-13, example 8.13b

Mehtane flow - Part "a" was compering to Bernoulli, which Arrow does not solve, so it was skipped. This model applies to part "b"

See Verify10.doc MS Word file for comparison with published results

Title: Verify10.aro - Methane flow in a pipe, Example 8.13b from Lindeburg page 8-12

Number Of Pipes $=1$

Number Of Junctions $=2$

Length March Solution Method with Mach Number Limits

Segments Per Pipe= 10

Mach Number Increment= 01

Preasure Tolerance $=.0001$ relative change

Mass Flow Rate Tolerance $=.0001$ relative change

Enthalpy Tolerance $=.0001$ relative change

Flow Relaxation $=.5$

Pressure Relaxation $=.5$

Fluid Database: AFT Standard

Fluid= Methane

Max Fluid Temperature Data $=\mathbf{4 0 0}$ deg. $K$

Min Fluid Temperature Data $=150$ deg. K

Molecular Weight= 16.043 amu

Gas Constant= $96.3243 \mathrm{ft}-1 \mathrm{~b} / \mathrm{hbm}-\mathrm{R}$

Critical Pressure= $4.5988 \mathrm{MPa}$

Critical Temperature= $190.555 \mathrm{deg} . \mathrm{K}$

Acentric Factor= .008

Equation of State= Redlich-Kwong

Enthaipy Model= Generalized

Specific Heat Ratio Accuracy= High

Atmospheric Pressure= $1 \mathrm{~atm}$

Gravitational Acceleration $=1 \mathrm{~g} / \mathrm{s}$

Standard Pressure= 14.696 paia

Standard Temperature $=60$ deg. F

Turbulent Flow Above Reynolds Number 4000

Laminar Flow Below Reynolds Number= 2300

Specific Heat Ratio Accuracy $=$ High

Atmospheric Pressure= $1 \mathrm{~atm}$

Gravitational Acceleration $=1$ o

Standard Pressure $=14.696$ psia

Standard Temperature $=60 \mathrm{deg} . F$

Turbulent Flow Above Reynolds Number $=4000$

Laminar Flow Below Reynolds Number= 2300

Pipe Input Table

\begin{tabular}{|c|r|r|r|r|r|r|r|r|r|r|}
\hline Pipe & Name & $\begin{array}{c}\text { Pipe } \\
\text { Defined }\end{array}$ & Length & $\begin{array}{c}\text { Length } \\
\text { Units }\end{array}$ & $\begin{array}{r}\text { Hydraulic } \\
\text { Diameter }\end{array}$ & $\begin{array}{c}\text { Hydraulic } \\
\text { Diam. Units }\end{array}$ & Roughness & $\begin{array}{c}\text { Roughness } \\
\text { Units }\end{array}$ & Losses (K) & Initial Flow \\
\hline 1 & Pipe & Yes & 75 & miles & 40 & inches & 0.00936 & Explicit $f$ & 0 & 0 \\
\hline
\end{tabular}

\begin{tabular}{|c|c|c|c|c|c|c|c|}
\hline Pipe & $\begin{array}{c}\text { Initial Flow } \\
\text { Units }\end{array}$ & $\begin{array}{c}\text { Junctions } \\
(\text { Up,Down })\end{array}$ & Geometry & Material & Size & Type & $\begin{array}{c}\text { Special } \\
\text { Condition }\end{array}$ \\
\hline 1 & & 1,2 & Cylindrical Pipe & Unsepecified & & & None \\
\hline
\end{tabular}

Assigned Pressure Table 
SNF-5331, Rev. 0

AFT Arrow 2.0 Input

(2)

$12 / 799$ 11:31 AM

Numatec Hanford Corporation

Verify 10.aro - Methane flow in a pipe, Example 8.13b from Lindeburg page 8-12

\begin{tabular}{|c|c|c|c|c|r|r|r|r|}
\hline Assigned Pressure & Name & $\begin{array}{c}\text { Object } \\
\text { Defined }\end{array}$ & $\begin{array}{c}\text { Database } \\
\text { Source }\end{array}$ & $\begin{array}{c}\text { Special } \\
\text { Condition }\end{array}$ & Fluid & Pressure & $\begin{array}{c}\text { Pressure } \\
\text { Units }\end{array}$ & \begin{tabular}{c} 
Temperature \\
\hline 1
\end{tabular} \\
\cline { 2 - 8 } & Assigned Pressure & Yes & & N/A & Methane & 650 & psia & 40 \\
\hline 2 & Assigned Pressure & Yes & & N/A & Methane & 450 & psia & 40 \\
\hline
\end{tabular}

\begin{tabular}{|c|r|r|}
\hline Assigned Pressure & $\begin{array}{r}\text { Temperature } \\
\text { Units }\end{array}$ & $\begin{array}{c}\text { Loss } \\
\text { Factor }\end{array}$ \\
\hline 1 & deg. F & 0 \\
\hline 2 & deg. F & 0 \\
\hline
\end{tabular}


Michael R. Lindeburg, P.E.

Mechanical Engineering Review Menual, Seventh Edition, Professional Publications, Belmont, CA, 1984

Pages 8-12, 8-13, example 8.13b

Mehtane flow - Part "a" was comparing to Bernoulli, which Artow does not solve, so it was skipped. This model applies to part "b"

See Verify 10.doc MS Word file for comparison with published reaults

Title: Verify 10.aro - Methane flow in a pipe, Example 8.13b from Lindeburg page 8-12

Analysis run on: 12/7/99 11:29:53 AM

Input File: C:LFT ProductsLAFT ArrowlVerificationlverify10.aro

Execution Time $=0.94$ seconds

Total Number Of Pressure Iterations $=0$

Total Number Of Flow lterations= 5

Total Number Of Enthalpy Iterations $=5$

Number Of Pipes= 1

Number Of Junctions= 2

Length March Solution Method with Mach Number Limits

Segments Per Pipe= 10

Mach Number Increment= .01

Pressure Tolerance= .0001 relative change

Mass Flow Rate Tolerancer .0001 relative change

Enthalpy Tolerancex .0001 relative change

Flow Relaxation $=.5$

Preasure Relaxation $=.5$

Fluid Database: AFT Standard

Fluid= Methane

Max Fluid Temperature Data $=400$ deg. $K$

Min Fluid Temperature Data $=150$ deg. K

Molecular Weight: $16.043 \mathrm{amu}$

Gas Constant= 96.3243 ft-lbf/bm-R

Critical Preasure $=4.5988 \mathrm{MPa}$

Critical Temperature $=190.555 \mathrm{deg} . \mathrm{K}$

Acentric Factorm .008

Equation of State= Redlich-Kwong

Enthalpy Model= Generalized

Specific Heat Ratio Accuracy= High

Atmospheric Pressure= 1 atm

Gravitational Acceleration= $1 \mathrm{~g} / \mathrm{s}$

Standard Pressure= 14.696 psia

Standard Temperature $=60 \mathrm{deg} . F$

Turbulent Flow Above Reynolds Number= 4000

Laminar Flow Below Reynolds Number= 2300

Specific Heat Ratio Accuracy= High

Atmospheric Pressure= $1 \mathrm{~atm}$

Gravitational Acceleration $=1 \mathrm{~g}$

Standard Pressure= 14.696 psia

Standard Temperature $=60$ deg. $F$

Turbulent Flow Above Reynolds Number $=\mathbf{4 0 0 0}$

Laminar Flow Below Reynolds Number= 2300

Overall Delta Preasure $=-199.926$ psia

Total Inflow= $478.029 \mathrm{lbm} / \mathrm{sec}$

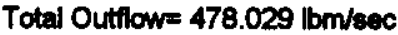

Total Energy Inflow= 182,233. Btu/s

Total Energy Outflowe 185,395. Btu/s

Total Heat Transferred Into System= 3161.81 Btu/s

Maximum Pressure is 650.000 paia at Junction 1 Inlet

Minimum Pressurs is 450.000 psia at Junction 2 Inlet

Maximum Static Temperature is $40.0463 \mathrm{deg}$. F at Junction 2 Inlet

Minimum Static Temperature is $\mathbf{4 0 . 0 1 8 7} \mathrm{deg}$. F at Junction 1 Inlet

Pipe Output Table 
SNF.5331, Rev. 0

AFT Arrow 2.0 Output

(2)

$127 / 99$

Numatec Hanford Corporation

Verify10.aro - Methane flow in a pipe, Example 8.13b from Lindeburg page 8-12

\begin{tabular}{|c|c|c|c|c|c|}
\hline Pipe & $\begin{array}{c}\text { Mass } \\
\text { Flow } \\
\text { (bm/sec) }\end{array}$ & Vel. In & Vel. Out & $\begin{array}{c}\text { P Static } \\
\text { In } \\
\text { (feet/sec) }\end{array}$ & $\begin{array}{c}\text { P Static } \\
\text { Out } \\
\text { (feet/sec) }\end{array}$ \\
\hline 1 & 478.029 & 25.3214 & 37.7986 & 650.000 & 449.971 \\
\hline
\end{tabular}

\section{All Junction Table}

\begin{tabular}{|c|c|c|}
\hline Jet & $\begin{array}{c}\text { P Static } \\
\text { In } \\
\text { (psia) }\end{array}$ & $\begin{array}{c}\text { P Static } \\
\text { Out } \\
\text { (psia) }\end{array}$ \\
\hline 1 & 650.000 & 650.000 \\
\hline 2 & 450.000 & 450.000 \\
\hline
\end{tabular}




\section{TITLE: VERIFY11.ARO}

REFERENCE: Michel A. Saad, Compressible Fluid Flow, 2nd Edition, Prentice-Hall, Englewood Cliffs, NJ, 1993, Pages 213-215, example 5.2

GAS: Air

ASSUMPTIONS: 1) Adiabatic, 2) Perfect gas

RESULTS:

\begin{tabular}{|l|r|r|}
\hline \multicolumn{1}{|c|}{ Parameter } & \multicolumn{1}{c|}{ Saad } & AFT Arrow 2.0 \\
\hline$M_{2}-$ Mach number at exit & 0.685 & 0.689 \\
\hline$M_{1}-$ Mach number at inlet & 0.347 & 0.347 \\
\hline$P_{1}-$ Static pressure at inlet (kPa) & 306 & 308.1 \\
\hline$T_{1}-$ Static temperature at inlet (deg. K) & 312.76 & 312.9 \\
\hline
\end{tabular}

\section{DISCUSSION:}

As specified, exit conditions are known and inlet conditions need to be determined for specified volume flow at exit. With the known exit conditions, an implied mass flow rate exists. To pose the problem in AFT Arrow terms, a few simple calculations are needed to obtain the mass flow rate. Once obtained, it is applied as a flow demand at the inlet.

The problem states that the exit volume flow rate, $Q_{2}$, is $1000 \mathrm{~m}^{3} / \mathrm{min}, P_{2}=150 \mathrm{kPa}, T_{2}=293 \mathrm{~K}$. From the ideal gas law, density, and mass flow rate are:

$$
\begin{aligned}
& \rho_{2}=\frac{P_{2}}{R T_{2}}=\frac{150 \mathrm{kPa}}{\left(.2868 \frac{\mathrm{kJ}}{\mathrm{kg}}\right)(293 \mathrm{~K})}=1.784 \mathrm{~kg} / \mathrm{m}^{3} \\
& \dot{m}=\rho_{2} V_{2} A=\rho_{2} Q_{2}=\left(1.784 \frac{\mathrm{kg}}{\mathrm{m}^{3}}\right)\left(1000 \frac{\mathrm{m}^{3}}{\mathrm{~min}}\right)=29.73 \mathrm{~kg} / \mathrm{s}
\end{aligned}
$$

In AFT Arrow, the discharge pressure can be specified. The temperature can be specified at the exit junction, but the actual discharge is what is displayed for the pipe exit. The pipe exit temperature depends on the inlet temperature and the thermodynamic process in the pipe, which is adiabatic. Therefore, to solve this problem the inlet static temperature at $\mathrm{J} 1$ must be guessed until the pipe delivers $293 \mathrm{~K}$ at the exit. This results in the $312.9 \mathrm{~K}$ displayed in the above table.

All results agree closely.

Note that the friction factor in Saad is the Fanning friction factor. To obtain the Darcy-Weisbach friction factor used in AFT Arrow, multiply the Fanning friction factor by 4.

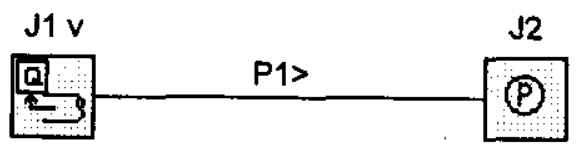

Copyright $\odot 1999$ Applied Flow Technology Corp. All rights reserved. 
SNF-5331, Rev. 0

AFT Arrow 2.0 Input

Numatec Hanford Corporation

(1)

12/7/99 11:46 AM

Verify11.aro - Air flow example 5.2 from Saad pp. 213-215

Michel A. Saad

Compressible Fluid Flow, 2nd Edition, Prentice-Hall, Englewood Cliffe, NJ, 1993

Page 213-215, example 5.2

Air flow - Example assumes adiabetic flow and air is a portect gas

See Verify 11. doc MS Word file for compariaon with published reaults

Title: Verify11.aro - Air flow example 5.2 from Saad pp. 213-215

Number Of Pipes $=1$

Number Of Junctions= 2

Length March Solution Method with Mach Number Limite

Segments Per Pipe $=10$

Mach Number Increment= 01

Pressure Tolerance= 0001 relative change

Mass Flow Rate Tolerance= .0001 relative change

Enthalpy Tolerancem .0001 relative change

Flow Relaxation= .5

Pressure Relaxation $=.5$

Fluid Database: AFT Standard

Fluid= Air

Max Fluid Temperature Data $=1000$ deg. $K$

Min Fluid Temperature Data $200 \mathrm{deg} . \mathrm{K}$

Molecular Woight $=28.97 \mathrm{amu}$

Gas Constant $=0.2868 \mathrm{~kJ} / \mathrm{ko}-\mathrm{K}$

Critical Pressure $=37.25 \mathrm{~atm}$

Critical Temperature $=132.41 \mathrm{deg} . \mathrm{K}$

Acentric Factor $=.021$

Equation of State= Ideal Gas

Enthalpy Model= Reference

Specific Heat Ratio Accuracy= High

Atmospheric Pressure= 1 atm

Gravitational Acceleration $=1$ g's

Standard Pressure= 14.696 peia

Standard Temperature $=60$ deg. F

Turbulent Flow Above Reynolds Number $\mathbf{4 0 0 0}$

Laminar Flow Below Reynolds Number= 2300

Specific Heat Ratio Accuracy= High

Atmospheric Pressures $1 \mathrm{~atm}$

Gravitational Acceleration $=1 \mathrm{~g}$

Standard Pressure $=14.696$ psia

Standard Temperature $=60$ deg. F

Turbulent Flow Above Reynolds Number $=4000$

Laminar Flow Below Reynolds Number $=\mathbf{2 3 0 0}$

Pipe Input Table

\begin{tabular}{|c|r|r|r|r|r|r|r|r|r|r|}
\hline Pipe & Name & $\begin{array}{c}\text { Pipe } \\
\text { Defined }\end{array}$ & Length & $\begin{array}{r}\text { Length } \\
\text { Units }\end{array}$ & $\begin{array}{r}\text { Hydraulic } \\
\text { Diameter }\end{array}$ & $\begin{array}{c}\text { Hydraulic } \\
\text { Diam. Units }\end{array}$ & Roughness & $\begin{array}{r}\text { Roughness } \\
\text { Units }\end{array}$ & Losses (K) & Initial Flow \\
\hline 1 & Pipe & Yes & 50 & meters & 0.3 & meters & 0.02 & Explicit f & 0 & \\
\hline
\end{tabular}

\begin{tabular}{|c|c|c|c|c|c|c|c|}
\hline Pipe & $\begin{array}{c}\text { Initial Flow } \\
\text { Units }\end{array}$ & $\begin{array}{c}\text { Junctions } \\
\text { (Up,Down) }\end{array}$ & Geometry & Meterial & Size & Type & $\begin{array}{c}\text { Special } \\
\text { Condition }\end{array}$ \\
\hline 1 & & 1,2 & Cylindrical Pipe & Unspecified & & & None \\
\hline
\end{tabular}

Assigned Flow Table 
SNF-5331, Rev. 0

AFT Arrow 2.0 input

(2)

12/7/99 11:46 AM

Numatec Hanford Corporation

Verify11.aro - Air flow example 5.2 from Saad pp. 213-215

\begin{tabular}{|c|c|c|c|c|c|c|c|c|c|}
\hline Assigned Flow & Name & $\begin{array}{c}\text { Object } \\
\text { Defined }\end{array}$ & $\begin{array}{c}\text { Database } \\
\text { Source }\end{array}$ & $\begin{array}{c}\text { Special } \\
\text { Condition }\end{array}$ & Fluid & Type & Flow & $\begin{array}{c}\text { Flow } \\
\text { Units }\end{array}$ & Temperature \\
\hline 1 & Assigned Flow & Yes & & None & Air & Inflow & 1783.961 & $\mathrm{~kg} / \mathrm{min}$ & 312.9 \\
\hline
\end{tabular}

\begin{tabular}{|c|r|r|}
\hline Assigned Flow & $\begin{array}{c}\text { Temperature } \\
\text { Units }\end{array}$ & $\begin{array}{c}\text { Loss } \\
\text { Model }\end{array}$ \\
\hline 1 & deg. $\mathrm{K}$ & 0 \\
\hline
\end{tabular}

Assigned Pressure Table

\begin{tabular}{|c|c|r|r|r|r|r|c|c|}
\hline Assigned Pressure & Name & $\begin{array}{c}\text { Object } \\
\text { Defined }\end{array}$ & $\begin{array}{c}\text { Database } \\
\text { Source }\end{array}$ & $\begin{array}{c}\text { Special } \\
\text { Condition }\end{array}$ & Fluid & Pressure & $\begin{array}{c}\text { Pressure } \\
\text { Units }\end{array}$ & \begin{tabular}{c} 
Temperature \\
\hline 2
\end{tabular} \\
\hline 2 & Assigned Pressure & Yes & & N/A & Air & 150 & $\mathrm{kPa}$ & 293 \\
\hline
\end{tabular}

\begin{tabular}{|c|r|r|}
\hline Assigned Pressure & $\begin{array}{c}\text { Temperature } \\
\text { Units }\end{array}$ & $\begin{array}{c}\text { Loss } \\
\text { Factor }\end{array}$ \\
\hline 2 & deg. $\mathrm{K}$ & 0 \\
\hline
\end{tabular}


Michel A. Saad

Compressible Fluid Flow, 2nd Edition, Prentice-Hall, Englowood Cliffe, NJ, 1993

Page 213-215, example 5.2

Air flow - Example assumes adiabetic flow and air is a perfect gas

See Verify11.doc MS Word file for comparison with published results

Titie: Verify11.aro - Air flow example 5.2 from Saad pp. 213-215

Analysis run on: $127 / 99$ 11:36:43 AM

Input File: C:AFT ProductsLAFT ArrowlVerificationiverify11,aro

Execution Time= 2.85 seconds

Total Number Of Pressure lterations= 0

Total Number Of Flow lterations= 2

Total Number Of Enthalpy Herations= 2

Number Of Pipes= 1

Number of Junctions= 2

Length March Solution Method with Mach Number Limits

Segments Per Pipe $=10$

Mach Number increment= .01

Preasure Tolerance= .0001 relative change

Mass Flow Rate Tolerance= .0001 relative change

Enthalpy Tolerance $=.0001$ relative change

Flow Relaxation $=.5$

Pressure Relaxation= .5

Fluid Database: AFT Standard

Fluid= Air

Max Fluid Temperature Data $=1000$ deg. $K$

Min Fluid Temperature Data $=200$ deg. K

Molecular Weight= $28.97 \mathrm{amu}$

Gas Constant $=0.2868 \mathrm{~kJ} / \mathrm{kg}-\mathrm{K}$

Critical Pressure $=37.25 \mathrm{~atm}$

Critical Temperature $=132.41 \mathrm{deg} . \mathrm{K}$

Acentric Factor= 021

Equation of State= Ideal Gas

Enthalpy Model= Reference

Specific Heat Ratio Accuracys High

Atmospheric Pressure= $1 \mathrm{~atm}$

Gravitational Acceleration $=1 \mathrm{~g}$ 's

Standard Pressure= 14.696 psia

Standard Temperature $=60 \mathrm{deg} . F$

Turbulent Flow Above Reynolds Number $=4000$

Laminar Flow Below Reynolds Number= $\mathbf{2 3 0 0}$

Specific Heat Ratio Accuracy= High

Atmospheric Pressure= $1 \mathrm{~atm}$

Gravitational Acceleration $=1 \mathrm{~g}$

Standard Preasure= 14.696 psia

Standard Temperature $=60 \mathrm{deg} . \mathrm{F}$

Turbulent Flow Above Reynolds Number= 4000

Leminar Flow Below Reynolds Numberm 2300

Overall Delta Pressure $=-128.9 \mathrm{kPa}$

Total Inflow= $29.73 \mathrm{~kg} / \mathrm{sec}$

Total Outflow= $29.73 \mathrm{~kg} / \mathrm{sec}$

Total Energy Inflow $14,287 \mathrm{~kW}$

Total Energy Outflow $=14,287 \mathrm{~kW}$

Total Heat Transferred Into System $=0.000 \mathrm{~kW}$

Maximum Pressure is $308.0 \mathrm{kPa}$ at Junction 1 Inlet

Minimum Pressure is $150.0 \mathrm{kPa}$ at Junction 2 Inlet

Maximum Static Temperature is $320.3 \mathrm{deg}$. K at Junction 1 iniet

Minimum Static Temperature is 293.0 deg. K at Junction 2 Inlet

Pipe Output Table 
Verify11.aro - Air flow example 5.2 from Saad pp. 213-215

\begin{tabular}{|c|c|c|c|c|c|c|c|c|c|c|}
\hline Pipe & $\begin{array}{c}\text { Mass } \\
\text { Flow } \\
(\mathrm{kg} / \mathrm{sec})\end{array}$ & $\begin{array}{c}\text { Vel. } \\
\text { In } \\
(\text { meters/sec) }\end{array}$ & $\begin{array}{c}\text { Vel. } \\
\text { Out } \\
\text { (meters/sec) }\end{array}$ & $\begin{array}{c}\text { Mach } \\
\text { In }\end{array}$ & $\begin{array}{c}\text { Mach } \\
\text { Out }\end{array}$ & $\begin{array}{c}\text { P Static } \\
\text { In } \\
(\mathrm{kPa})\end{array}$ & $\begin{array}{c}\text { P Static } \\
\text { Out } \\
(\mathrm{kPa})\end{array}$ & $\begin{array}{c}\text { T Static } \\
\text { In } \\
\text { (deg. K) }\end{array}$ & $\begin{array}{c}\text { T Static } \\
\text { Out } \\
\text { (deg. K) }\end{array}$ & $\begin{array}{c}\text { Rho Static } \\
\text { Out } \\
(\mathrm{kg} / \mathrm{m} 3)\end{array}$ \\
\hline 1 & 29.73 & 122.6 & 235.8 & 0.3469 & 0.6891 & 308.1 & 150.0 & 312.9 & 293.0 & 1.784 \\
\hline
\end{tabular}

All Junction Table

\begin{tabular}{|c|c|c|r|r|}
\hline Jet & $\begin{array}{c}\text { P Static } \\
\text { In } \\
(\mathrm{KPa})\end{array}$ & $\begin{array}{c}\text { P Static } \\
\text { Out } \\
(\mathrm{KPa})\end{array}$ & $\begin{array}{c}\text { T Static } \\
\text { In } \\
(\text { deq. } \mathrm{K})\end{array}$ & $\begin{array}{c}\text { T Static } \\
\text { Out } \\
\text { (deq. K) }\end{array}$ \\
\hline 1 & 308.0 & 308.0 & 312.9 & 312.9 \\
\hline 2 & 150.0 & 150.0 & 268.0 & 268.0 \\
\hline
\end{tabular}


TITLE: VERIFY12.ARO

REFERENCE: Michel A. Saad, Compressible Fluid Flow, 2nd Edition, Prentice-Hall, Englewood Cliffs, NJ, 1993, Pages 226-227, example 5.5

GAS: Air

ASSUMPTIONS: 1) Adiabatic, 2) Perfect gas

RESULTS:

\begin{tabular}{|l|r|r|}
\hline \multicolumn{1}{|c|}{ Parameter } & \multicolumn{1}{c|}{ Saad } & AFT Arrow 2.0 \\
\hline Mass flow rate when choked (kg/s) & 2.11 & 2.10 \\
\hline$M_{1}-$ Mach number at inlet & 0.603 & 0.604 \\
\hline$P_{1}-$ Static pressure at inlet (MPa) & 2.106 & 2.114 \\
\hline$T_{1}-$ Static temperature at inlet (deg. K) & 427.8 & 429.6 \\
\hline$P_{2, \text { choke }}$ - Static back pressure for choking (MPa) & 1.203 & 1.217 \\
\hline
\end{tabular}

\section{DISCUSSION:}

All results agree closely. The AFT Arrow static pressure below which choking occurs is the pipe exit static pressure.

Note that the friction factor in Saad is the Fanning friction factor. To obtain the Darcy-Weisbach friction factor used in AFT Arrow, multiply the Fanning friction factor by 4.

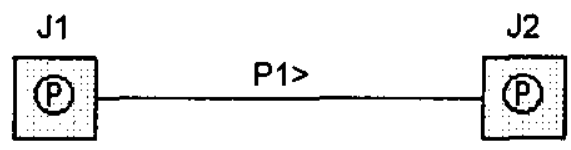

Copyright $(1999$ Applied Flow Technology Corp. All rights reserved. 
Michel A. Saad

Compressible Fluid Flow, 2nd Edition, Prentice-HaH, Englewood Cliffa, NJ, 1993

Page 226-227, example 5.5

Air flow - Example assumes adiabatic flow and air is a perfect gas

See Verify12.doc MS Word file for comparison with publiahed results

Title: Verify 12.aro - Air flow example 5.5 from Saad pp. 226-227

Number Of Pipes= 1

Number Of Junctions= 2

Length March Solution Method with Mach Number Limits

Segments Per Pipe= 10

Mach Number increment= 01

Pressure Tolerances .0001 relative change

Mass Flow Rate Tolerance $=0001$ relative change

Enthalpy Tolerances .0001 relative change

Flow Relaxation= .5

Preasure Relaxation $=.5$

Fluid Database: AFT Standard

Fluid= Air

Max Fluid Temperature Data $=1000$ deg. $K$

Min Fluid Temperature Data $=200$ deg. K

Molocular Woight= $28.97 \mathrm{amu}$

Gas Constant $=0.2868 \mathrm{~kJ} / \mathrm{kg}-\mathrm{K}$

Critical Pressure $=37.25 \mathrm{~atm}$

Critical Temperature= 132.41 deg. $K$

Acentric Factor $=021$

Equation of State= Ideal Gas

Enthalpy Model= Reference

Specific Heat Ratio Accuracy = High

Atmospheric Pressure $=1 \mathrm{~atm}$

Gravitational Acceleration $=1 \mathrm{~g} / \mathrm{s}$

Standard Pressure $=14.696$ paia

Standard Temperature $=60 \mathrm{deg}$. F

Turbulent Flow Above Reynolds Number $=4000$

Laminar Flow Below Reynolds Number $=\mathbf{2 3 0 0}$

Specific Heat Ratio Accuracy= High

Atmospheric Pressure $=1$ atm

Gravitational Acceleration $=1 \mathrm{~g}$

Standard Pressuren 14.696 psia

Standard Temperature= $60 \mathrm{deg}$. F

Turbulent Flow Above Reynolds Number $=4000$

Laminar Flow Below Reynolds Number= 2300

Pipe Input Table

\begin{tabular}{|c|r|r|r|r|r|r|r|r|r|r|}
\hline Pipe & Name & $\begin{array}{c}\text { Pipe } \\
\text { Defined }\end{array}$ & Length & $\begin{array}{r}\text { Length } \\
\text { Units }\end{array}$ & $\begin{array}{r}\text { Hydraulic } \\
\text { Diameter }\end{array}$ & $\begin{array}{c}\text { Hydraulic } \\
\text { Diam. Units }\end{array}$ & Roughness & $\begin{array}{c}\text { Roughness } \\
\text { Units }\end{array}$ & Losses (K) & Initial Flow \\
\hline 1 & Pipe & Yes & 0.6 & metere & $\mathbf{0 . 0 2 5}$ & meters & 0.02 & Explicitf & 0 & \\
\hline
\end{tabular}

\begin{tabular}{|c|c|c|c|c|c|c|c|}
\hline Pipe & $\begin{array}{c}\text { Initial Flow } \\
\text { Units }\end{array}$ & $\begin{array}{c}\text { Junctions } \\
\text { (Up.Down })\end{array}$ & Geometry & Material & Size & Type & $\begin{array}{c}\text { Special } \\
\text { Condition }\end{array}$ \\
\hline 1 & & 1,2 & Cylindrical Pipe & Unepecified & & & None \\
\hline
\end{tabular}

\section{Assigned Pressure Table}


SNF-5331, Rev. 0

AFT Arrow 2.0 input

(2)

Numatec Hanford Corporation

Verify12.aro - Air flow example 5.5 from Saad pp. 226-227

\begin{tabular}{|c|c|r|r|r|r|r|r|r|}
\hline Assigned Pressure & Name & $\begin{array}{c}\text { Object } \\
\text { Defined }\end{array}$ & $\begin{array}{c}\text { Database } \\
\text { Source }\end{array}$ & $\begin{array}{c}\text { Special } \\
\text { Condition }\end{array}$ & Fluid & Pressure & $\begin{array}{c}\text { Pressure } \\
\text { Units }\end{array}$ & Temperature \\
\hline 1 & Assigned Pressure & Yes & & N/A & Air & 2.7 & $\mathrm{MPa}$ & 460 \\
\hline 2 & Assigned Pressure & Yes & & N/A & Air & 0.1 & $\mathrm{MPa}$ & 293 \\
\hline
\end{tabular}

\begin{tabular}{|c|r|r|}
\hline Assigned Pressure & $\begin{array}{r}\text { Temperature } \\
\text { Units }\end{array}$ & $\begin{array}{c}\text { Loss } \\
\text { Factor }\end{array}$ \\
\hline 1 & deg. K & 0 \\
\hline 2 & deg. K & 0 \\
\hline
\end{tabular}


Michel A. Saad

Compressible Fluid Flow, 2nd Edition, Prentice-Hall, Englewood Cliffs, NJ, 1993

Page 226-227, example 5.5

Air flow - Example assumes adiabatic flow and air is a perfect gas

See Verify12.doc MS Word file for comparison with publiahed results

Title: Verify12.aro - Air flow example 5.5 from Saad pp. 226-227

Analysis run on: 12/7/99 11:50:14 AM

Input File: C:AFT ProductsVAFT ArrowlVerificationiverify12.aro

Execution Time $=1.43$ seconds

Total Number Of Pressure Iterations $=0$

Total Number Of Flow lterations $=2$

Total Number Of Enthalpy Iterations= 2

Number Of Pipes= 1

Number Of Junctions= 2

Length March Solution Method with Mach Number Limits

Segments Per Pipe= 10

Mach Number Increment= 01

Pressure Tolerance $=.0001$ relative change

Mass Flow Rate Tolerance= .0001 relative change

Enthalpy Tolerance $=.0001$ relative change

Flow Relaxation $=.5$

Pressure Relaxation $=.5$

Fluid Database: AFT Standard

Fluid= Air

Max Fluid Temperature Data $=1000 \mathrm{deg} . \mathrm{K}$

Min Fluid Temperature Data $=200 \mathrm{deg} . \mathrm{K}$

Molecular Weight $=28.97$ amu

Gas Constant= $0.2868 \mathrm{~kJ} / \mathrm{kg}-\mathrm{K}$

Critical Pressure $=37.25 \mathrm{~atm}$

Critical Temperature $=132.41$ deg. $\mathrm{K}$

Acentric Factor $=.021$

Equation of State= Ideal Gas

Enthalpy Model= Reference

Specific Heat Ratio Accuracy= High

Atmospheric Pressures $1 \mathrm{~atm}$

Gravitational Acceleration $=1 \mathrm{~g}$ 's

Standard Pressure= 14.696 psia

Standard Temperature $=60 \mathrm{deg} . \mathrm{F}$

Turbulent Flow Above Reynolds Number= 4000

Laminar Flow Below Reynolds Number= 2300

Specific Heat Ratio Accuracy= High

Atmospheric Pressure= $1 \mathrm{~atm}$

Gravitational Acceleration $=1 \mathrm{~g}$

Standard Pressure= $14.696 \mathrm{psia}$

Standard Temperature 60 deg. $F$

Turbulent Flow Above Reynolds Number $=\mathbf{4 0 0 0}$

Laminar Flow Below Reynolds Number $=\mathbf{2 3 0 0}$

Overall Delta Pressure $=-\mathbf{2 6 0 0}$. kPa

Total Inflow= $2.103 \mathrm{~kg} / \mathrm{sec}$

Total Outflow $=2.103 \mathrm{~kg} / \mathrm{sec}$

Total Energy inflow= 1311. kW

Total Energy Outflow= 1311. kW

Total Heat Transferred Into System $=-2.662 \mathrm{E}-06 \mathrm{~kW}$

Maximum Pressure is 2280. kPa at Junction 2 Inlet

Minimum Pressure is $100.0 \mathrm{kPa}$ at Junction 2 Outlet

Maximum Static Temperature is $460.0 \mathrm{deg}$. K at Junction 1 Inlet

Minimum Static Temperature is $293.0 \mathrm{deg}$. K at Junction 2 Inlet

".CAUTION"** Junction 2 is downstream of a shock, static properties cannot be calculated at junction.

Sonic Choking Exists at Junction 2 (Assigned Pressure) 
SNF-5331, Rev. 0

Verify 12.aro - Air flow example 5.5 from Saad pp. 226-227

\begin{tabular}{|c|c|c|c|c|c|c|c|c|c|}
\hline Pipe & $\begin{array}{c}\text { Mass } \\
\text { Flow } \\
(\mathrm{kg} / \mathrm{sec})\end{array}$ & $\begin{array}{c}\text { Vet. } \\
\text { In } \\
(\text { meters/sec) }\end{array}$ & $\begin{array}{c}\text { Vel. } \\
\text { Out } \\
(\text { metera/sec) }\end{array}$ & $\begin{array}{c}\text { Mach } \\
\text { In }\end{array}$ & $\begin{array}{c}\text { Mach } \\
\text { Out }\end{array}$ & $\begin{array}{c}\text { P Static } \\
\text { In } \\
(\mathrm{MPa})\end{array}$ & $\begin{array}{c}\text { P Static } \\
\text { Out } \\
(\mathrm{MPa})\end{array}$ & $\begin{array}{c}\text { T Static } \\
\text { In } \\
(\text { deg. K) }\end{array}$ & $\begin{array}{c}\text { T Static } \\
\text { Out } \\
\text { (deg. K) })\end{array}$ \\
\hline 1 & 2.103 & 249.8 & 389.9 & 0.6039 & 0.9939 & 2.114 & 1.217 & 429.6 & 386.0 \\
\hline
\end{tabular}

\section{All Junction Table}

\begin{tabular}{|c|c|r|r|r|}
\hline Jct & $\begin{array}{c}\text { P Static } \\
\text { In } \\
(\mathrm{kPa})\end{array}$ & $\begin{array}{c}\text { P Static } \\
\text { Out } \\
\text { (kPa) }\end{array}$ & $\begin{array}{c}\text { T Static } \\
\text { In } \\
\text { (deq. K) }\end{array}$ & $\begin{array}{c}\text { T Static } \\
\text { Out } \\
\text { (deg. } \mathrm{K})\end{array}$ \\
\hline 1 & 2114 & 2114.3 & 429.6 & 429.6 \\
\hline 2 & 2280 & 100.0 & 293.0 & 293.0 \\
\hline
\end{tabular}




\section{TITLE: VERIFY13.ARO}

REFERENCE: Michel A. Saad, Compressible Fluid Flow, 2nd Edition, Prentice-Hall, Englewood Cliffs, NJ, 1993, Page 270, example 6.5

GAS: Natural Gas

ASSUMPTIONS: 1) Isothermal, 2) Perfect gas, 3) Natural gas can be represented with methane RESULTS:

\begin{tabular}{|l|r|r|}
\hline \multicolumn{1}{|c|}{ Parameter } & \multicolumn{1}{c|}{ Saad } & AFT Arrow 2.0 \\
\hline$P_{2}-$ Static pressure at exit (kPa) & 263.16 & 262.0 \\
\hline$M_{2}-$ Mach number at exit & 0.38 & 0.3818 \\
\hline$L_{1-2}-$ Length to reach $500 \mathrm{kPa}(\mathrm{m})$ & 544.79 & 558.5 \\
\hline$L_{\mathrm{T}}^{*}-$ Length to reach sonic choke point $(\mathrm{m})$ & 710 & 709.8 \\
\hline$P_{\mathrm{T}}{ }^{*}-$ Static pressure choke point $(\mathrm{kPa})$ & 114.46 & 114.9 \\
\hline$M_{\mathrm{T}}^{*}-$ Mach number at isothermal choke point & 0.874 & 0.8704 \\
\hline
\end{tabular}

\section{DISCUSSION:}

As specified, inlet conditions are known and outlet conditions need to be determined. With the known inlet conditions, an implied mass flow rate exists. To pose the problem in AFT Arrow terms, a few simple calculations are needed to obtain the mass flow rate. Once obtained, it is applied as a flow demand at the exit.

The problem states that the inlet Mach number is $0.1, P_{1}=1 \mathrm{MPa}, T_{1}=293 \mathrm{~K}$. From the ideal gas law, density, sonic speed and mass flow rate are:

$$
\begin{aligned}
& \rho_{1}=\frac{P_{1}}{R T_{1}}=\frac{1 \mathrm{MPa}}{\left(.5179 \frac{\mathrm{kJ}}{\mathrm{kg} \cdot \mathrm{K}}\right)(293 \mathrm{~K})}=6.585 \mathrm{~kg} / \mathrm{m}^{3} \\
& a_{1}=\sqrt{\gamma R T_{1}}=\sqrt{1.4\left(.5179 \frac{\mathrm{kJ}}{\mathrm{kg} \cdot \mathrm{K}}\right) 293 \mathrm{~K}}=445.9 \mathrm{~m} / \mathrm{s} \text { (sonic velocity) } \\
& \dot{m}=\rho_{1} V_{1} A=\rho_{1}\left(M_{1} a_{1}\right) A=\left(6.585 \frac{\mathrm{kg}}{\mathrm{m}^{3}}\right)(0.1)\left(445.9 \frac{\mathrm{m}}{\mathrm{s}}\right)\left(\frac{\pi}{4} 0.08^{2} \mathrm{~m}^{2}\right)=1.4764 \mathrm{~kg} / \mathrm{s}
\end{aligned}
$$

The first pipe represents the pipe from point 1 to 2 . The second pipe represents the pipe from point 1 to the choking pint. AFT Arrow does not solve for pipe length. To obtain the maximum pipe length, different lengths must be guessed with lengths that exceed sonic flow discarded.

All results agree closely. The AFT Arrow static pressure below which choking occurs is the pipe exit static pressure.

Note that the friction factor in Saad is the Fanning friction factor. To obtain the Darcy-Weisbach friction factor used in AFT Arrow, multiply the Fanning friction factor by 4.

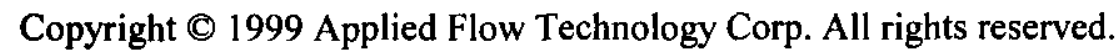


SNF-5331, Rev. 0

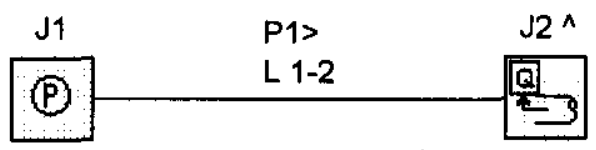

\begin{tabular}{|c|c|}
\hline J3 & P2> \\
\hline (P) & L1-Choke \\
\hline
\end{tabular}


Michel A. Saad

Compressible Fluid Flow, 2nd Edition, Prentice-Hall, Englewood Cliffs, NJ, 1993

Page 270, example 6.5

Natural Gas flow - Isothermal flow, perfect gas, assumed to be methane

See Verify 13.doc MS Word file for comparison with published results

Title: Verify13.aro Natural Gas flow example 6.5 from Saad pp. 270

Number of Pipes= 2

Number of Junctions $=4$

Length March Solution Method with Mach Number Limits

Segments Per Pipe= $\mathbf{5 0}$

Mach Number Increment= .01

Pressure Tolerance .0001 relative change

Mass Flow Rate Tolerance= .0001 relative change

Enthalpy Tolerancer .0001 relative change

Flow Relaxation $=.5$

Pressure Relaxation= .5

Fluid Database: AFT Standard

Fluid= Methane

Max Fluid Temperature Data $=400 \mathrm{deg} . \mathrm{K}$

Min Fluid Temperature Data $=150$ deg. K

Molecular Weight $=16.043 \mathrm{amu}$

Gas Constant $=0.5179 \mathrm{~kJ} / \mathrm{kg}-\mathrm{K}$

Critical Presesure $=4.5988 \mathrm{MPa}$

Critical Temperature $=190.555$ deg. $K$

Acentric Factor $=.008$

Equation of State= Ideal Gas

Enthalpy Model= Reference

Specific Heat Ratio Accuracy= High

Atmospheric Pressure= $1 \mathrm{~atm}$

Gravitational Acceleration $=1$ g's

Standard Pressure= $0.1 \mathrm{MPa}$

Standard Temperature $=293 \mathrm{deg} . \mathrm{K}$

Turbulent Flow Above Reynolds Number $=4000$

Laminar Flow Below Reynolds Number $=2300$

Specific Heat Ratio Accuracy= High

Atmoapheric Pressure= $1 \mathrm{~atm}$

Gravitational Acceleration= $1 \mathrm{~g}$

Standard Pressure $=0.1 \mathrm{MPa}$

Standard Temperature $=293$ deg. K

Turbulent Flow Above Reynolds Number $=4000$

Laminar Flow Below Reynolds Number $=\mathbf{2 3 0 0}$

Pipe Input Table

\begin{tabular}{|c|c|c|c|c|c|c|c|c|c|c|}
\hline Pipe & Name & $\begin{array}{c}\text { Pipe } \\
\text { Defined }\end{array}$ & Length & $\begin{array}{l}\text { Length } \\
\text { Units }\end{array}$ & $\begin{array}{l}\text { Hydraulic } \\
\text { Diameter }\end{array}$ & $\begin{array}{c}\text { Hydraulic } \\
\text { Diam. Units }\end{array}$ & Roughness & $\begin{array}{c}\text { Roughness } \\
\text { Units }\end{array}$ & Losses (K) & Initial Flow \\
\hline 1 & $\llcorner 1-2$ & Yes & 684 & meters & 0.08 & meters & 0.008 & Explicit $f$ & 0 & \\
\hline 2 & L1-Choke & Yes & 709.814 & meters & 0.08 & meters & 0.008 & Explicit f & 0 & \\
\hline
\end{tabular}

\begin{tabular}{|c|r|r|c|c|c|c|c|}
\hline Pipe & $\begin{array}{c}\text { Initial Flow } \\
\text { Units }\end{array}$ & $\begin{array}{c}\text { Junctions } \\
\text { (Up,Down) }\end{array}$ & Geometry & Material & Size & Type & $\begin{array}{c}\text { Special } \\
\text { Condition }\end{array}$ \\
\hline 1 & & 1,2 & Cylindrical Pipe & Unspecified & & & None \\
\hline 2 & & 3,4 & Cylindrical Pipe & Unspecified & & & None \\
\hline
\end{tabular}


SNF-5331, Rev. 0

AFT Arrow 2.0 Input

(2)

12/799 11:59 AM

Numatec Hanford Corporation

Verify13.aro Natural Gas flow example 6.5 from Saad pp. 270

\begin{tabular}{|c|c|c|c|r|r|r|r|r|r|}
\hline Assigned Flow & Name & $\begin{array}{c}\text { Object } \\
\text { Defined }\end{array}$ & $\begin{array}{c}\text { Database } \\
\text { Source }\end{array}$ & $\begin{array}{c}\text { Special } \\
\text { Condition }\end{array}$ & Fluid & Type & Flow & $\begin{array}{c}\text { Flow } \\
\text { Units }\end{array}$ & Temperature \\
\hline 2 & Assigned Flow & Yes & & None & Methane & Outflow & 1.4764 & $\mathrm{~kg} / \mathrm{sec}$ & 293 \\
\hline 4 & Assigned Flow & Yes & & None & Methane & Outflow & 1.4764 & $\mathrm{~kg} / \mathrm{sec}$ & \\
\hline
\end{tabular}

\begin{tabular}{|c|r|r|}
\hline Assigned Flow & $\begin{array}{r}\text { Temperature } \\
\text { Units }\end{array}$ & $\begin{array}{c}\text { Loss } \\
\text { Model }\end{array}$ \\
\hline 2 & deg. $\mathrm{K}$ & 0 \\
\hline 4 & deg. $\mathrm{K}$ & 0 \\
\hline
\end{tabular}

\section{Assigned Pressure Table}

\begin{tabular}{|c|c|r|r|r|r|r|r|r|}
\hline Assigned Pressure & Name & $\begin{array}{c}\text { Object } \\
\text { Defined }\end{array}$ & $\begin{array}{c}\text { Database } \\
\text { Source }\end{array}$ & $\begin{array}{c}\text { Special } \\
\text { Condition }\end{array}$ & Fluid & Pressure & $\begin{array}{c}\text { Pressure } \\
\text { Units }\end{array}$ & \begin{tabular}{c} 
Temperature \\
\hline 1
\end{tabular} \\
\hline $\mathbf{3}$ & Assigned Pressure & Yes & & N/A & Methane & 1 & $\mathrm{MPa}$ & 293 \\
\hline
\end{tabular}

\begin{tabular}{|c|c|r|}
\hline Assigned Pressure & $\begin{array}{c}\text { Temperature } \\
\text { Units }\end{array}$ & $\begin{array}{c}\text { Loss } \\
\text { Factor }\end{array}$ \\
\hline 1 & deg. K & 0 \\
\hline 3 & deg. $K$ & 0 \\
\hline
\end{tabular}


Michel A. Saad

Compressible Fluid Flow, 2nd Edition, Prentice-Hall, Englewood Cliffs, NJ, 1993

Page 270, example 6.5

Natural Gas flow - lsothermal flow, perfect gas, aseumed to be methane

See Verify13.doc MS Word file for comparison with published results

Title: Verify 13.aro Natural Gas flow example 6.5 from Saad pp. 270

Analysis run on: 12/7199 11:55:34 AM

Input File: C:LFT ProductsLAFT ArroutVerificationiverify13.aro

Execution Time $=1.60$ seconds

Total Number of Pressure Iterations= 0

Total Number Of Flow lterations= 2

Total Number of Enthalpy lterations $=2$

Number Of Pipes= 2

Number Of Junctions $=4$

Length March Solution Method with Mach Number Limits

Segments Per Pipe $=50$

Mach Number Increment= 01

Pressure Tolerances .0001 retative change

Mass Flow Rate Tolerance .0001 relative change

Enthalpy Tolerance $=.0001$ relative change

Flow Relaxation $=.5$

Pressure Relaxation $=.5$

Fluid Database: AFT Standard

Fluid= Methane

Max Fluid Temperature Data $=\mathbf{4 0 0}$ deg. K

Min Fluid Temperature Data $=150$ deg. K

Molecular Weight= $16.043 \mathrm{amu}$

Gas Constant $=0.5179 \mathrm{~kJ} / \mathrm{kg}-\mathrm{K}$

Critical Pressure= $4.5988 \mathrm{MPa}$

Critical Temperature $=190.555 \mathrm{deg} . \mathrm{K}$

Acentric Factor $=.008$

Equation of Staten Ideal Gas

Enthalpy Model= Reference

Specific Heat Ratio Accuracy= High

Atmospheric Pressure= $1 \mathrm{~atm}$

Gravitational Acceleration $=1 \mathrm{~g}$

Standard Pressure $=0.1 \mathrm{MPa}$

Standard Temperature $=293$ deg. $\mathrm{K}$

Turbulent Flow Above Reynolds Number $=4000$

Laminar Flow Bolow Reynolds Number= 2300

Specific Heat Ratio Accuracy= High

Atmospheric Pressure= $1 \mathrm{~atm}$

Gravitational Acceleration $=1 \mathrm{~g}$

Standard Pressure $=0.1 \mathrm{MPa}$

Standard Temperature $=\mathbf{2 9 3}$ deg. $\mathrm{K}$

Turbulent Flow Above Reynolds Number $=\mathbf{4 0 0 0}$

Laminar Flow Below Reynolds Number= 2300

Total inflow $=2.953 \mathrm{~kg} / \mathrm{sec}$

Total Outflow $=2.953 \mathrm{~kg} / \mathrm{sec}$

Total Energy Inflow= 2865. kW

Total Energy Outflow= 2995. kW

Total Heat Transferred Into System $=129.6 \mathrm{~kW}$

Maximum Pressure is $1.000 \mathrm{MPa}$ at Junction 1 Inlet Minimum Pressure is $0.1149 \mathrm{MPa}$ at Junction 4 Inlet

Maximum Static Temperature is 54.17 deg. C at Junction 4 Inlet Minimum Static Temperature is 20.30 deg. $C$ at Junction 1 Inlet

Pipe Output Table 
SNF-5331, Rev. 0

AFT Arrow 2.0 Output

Numatec Hanford Corporation

(2)

$12 / 7 / 99$

Verify13.aro Natural Gas flow example 6.5 from Saad pp. 270

\begin{tabular}{|c|r|r|r|r|r|r|r|r|r|r|r|r|}
\hline Pipe & Name & Length & $\begin{array}{c}\text { Mass } \\
\text { Flow } \\
\text { (meters) } \\
(\mathrm{kg} / \mathrm{sec})\end{array}$ & $\begin{array}{c}\text { Mach } \\
\text { \# In }\end{array}$ & $\begin{array}{c}\text { Mach } \\
\text { \# Out }\end{array}$ & $\begin{array}{c}\text { P Static } \\
\text { In } \\
(\mathrm{kPa})\end{array}$ & $\begin{array}{c}\text { P Static } \\
\text { Out } \\
(\mathrm{kPa})\end{array}$ & $\begin{array}{c}\text { T Static } \\
\text { In } \\
(\text { deg. K) }\end{array}$ & $\begin{array}{c}\text { T Static } \\
\text { Out } \\
\text { (deg. K) }\end{array}$ & $\begin{array}{c}\text { Gamma } \\
\text { In }\end{array}$ & $\begin{array}{c}\text { Gamma } \\
\text { Out }\end{array}$ & $\begin{array}{r}\text { Rho } \\
\text { Static In } \\
(\mathrm{kg} / \mathrm{m} 3)\end{array}$ \\
\hline 1 & L 1-2 & 684.0 & 1.476 & 0.1000 & 0.3818 & 1000 & 262.0 & 293.0 & 293.0 & 1.309 & 1.309 & 6.585 \\
\hline 2 & L1-Choke & 709.8 & 1.476 & 0.1000 & 0.8704 & 1000 & 114.9 & 293.0 & 293.0 & 1.309 & 1.309 & 6.585 \\
\hline
\end{tabular}

\begin{tabular}{|c|c|r|r|}
\hline Pipe & $\begin{array}{c}\text { Rho Static } \\
\text { Out } \\
(\mathrm{kg} / \mathrm{m} 3)\end{array}$ & $\begin{array}{c}\text { Vel. } \\
\text { Sonic In } \\
(\text { meterg/sec) }\end{array}$ & $\begin{array}{c}\text { Vel. } \\
\text { Sonic Out } \\
(\mathrm{meters} / \mathrm{sec})\end{array}$ \\
\hline 1 & 1.7253 & 445.9 & 445.9 \\
\hline 2 & 0.7568 & 445.9 & 445.9 \\
\hline
\end{tabular}

Internal Pipe Results, Pipe 1, Name: L 1-2

\begin{tabular}{|c|c|c|c|c|c|c|c|c|}
\hline Point & $\begin{array}{c}x \\
\text { (meters) } \\
\end{array}$ & $\begin{array}{c}\text { Mass } \\
\text { Flow } \\
(\mathrm{kg} / \mathrm{soc})\end{array}$ & $\begin{array}{c}\text { Mach } \\
\#\end{array}$ & $\begin{array}{c}P \\
\text { Static } \\
(\mathrm{kPa})\end{array}$ & $\begin{array}{c}T \\
\text { Static } \\
\text { (deg. K) } \\
\end{array}$ & Gamma & $\begin{array}{c}\text { Rho } \\
\text { Static } \\
(\mathrm{kg} / \mathrm{m} 3)\end{array}$ & $\begin{array}{c}\text { Vel. } \\
\text { Sonic } \\
\text { (meters/sec) }\end{array}$ \\
\hline 1 & 0.00 & 1.476 & 0.1000 & 1000.0 & 293.0 & 1.309 & 6.585 & 445.9 \\
\hline 2 & 13.68 & 1.476 & 0.1009 & 990.9 & 293.0 & 1.309 & 6.525 & 445.9 \\
\hline 3 & 27.36 & 1.476 & 0.1019 & 981.7 & 293.0 & 1.309 & 6.465 & 445.9 \\
\hline 4 & 41.04 & 1.476 & 0.1029 & 972.4 & 293.0 & 1.309 & 6.404 & 445.9 \\
\hline 5 & 54.72 & 1.476 & 0.1039 & 963.0 & 293.0 & 1.309 & 6.342 & 445.9 \\
\hline 6 & 68.40 & 1.476 & 0.1049 & 953.5 & 293.0 & 1.309 & 6.279 & 445.9 \\
\hline 7 & 82.08 & 1.476 & 0.1060 & 943.9 & 293.0 & 1.309 & 6.216 & 445.9 \\
\hline 8 & 95.76 & 1.476 & 0.1071 & 934.2 & 293.0 & 1.309 & 6.152 & 445.9 \\
\hline 9 & 109.44 & 1.476 & 0.1082 & 924.4 & 293.0 & 1.309 & 6.088 & 445.9 \\
\hline 10 & 123.12 & 1.476 & 0.1094 & 914.5 & 293.0 & 1.309 & 6.023 & 445.9 \\
\hline 11 & 136.80 & 1.476 & 0.1106 & 904.5 & 293.0 & 1.309 & 5.957 & 445.9 \\
\hline 12 & 150.48 & 1.476 & 0.1118 & 894.4 & 293.0 & 1.309 & 5.890 & 445.9 \\
\hline 13 & 164.16 & 1.476 & 0.1131 & 884.1 & 293.0 & 1.309 & 5.823 & 445.9 \\
\hline 14 & 177.84 & 1.476 & 0.1145 & 873.8 & 293.0 & 1.309 & 5.754 & 445.9 \\
\hline 15 & 191.52 & 1.476 & 0.1159 & 863.3 & 293.0 & 1.309 & 5.685. & 445.9 \\
\hline 16 & 205.20 & 1.476 & 0.1173 & 852.6 & 293.0 & 1.309 & 5.615 & 445.9 \\
\hline 17 & 218.88 & 1.476 & 0.1188 & 841.9 & 293.0 & 1.309 & 5.544 & 445.9 \\
\hline 18 & 232.56 & 1.476 & 0.1204 & 830.9 & 293.0 & 1.309 & 5.472 & 445.9 \\
\hline 19 & 246.24 & 1.476 & 0.1220 & 819.9 & 293.0 & 1.309 & 5.399 & 445.9 \\
\hline 20 & 259.92 & 1.476 & 0.1237 & 808.7 & 293.0 & 1.309 & 5.325 & 445.9 \\
\hline 21 & 273.60 & 1.476 & 0.1255 & 797.3 & 293.0 & 1.309 & 5.250 & 445.9 \\
\hline 22 & 287.28 & 1.476 & 0.1273 & 785.7 & 293.0 & 1.309 & 5.174 & 445.9 \\
\hline 23 & 300.96 & 1.476 & 0.1292 & 773.9 & 293.0 & 1.309 & 5.097 & 445.9 \\
\hline 24 & 314.64 & 1.476 & 0.1313 & 762.0 & 293.0 & 1.309 & 5.018 & 445.9 \\
\hline 25 & 328.32 & 1.476 & 0.1334 & 749.9 & 293.0 & 1.309 & 4.938 & 445.9 \\
\hline 26 & 342.00 & 1.476 & 0.1356 & 737.5 & 293.0 & 1.309 & 4.857 & 445.9 \\
\hline 27 & 355.68 & 1.476 & 0.1380 & 725.0 & 293.0 & 1.309 & 4.774 & 445.9 \\
\hline 28 & 369.36 & 1.476 & 0.1405 & 712.2 & 293.0 & 1.309 & 4.690 & 445.9 \\
\hline 29 & 383.04 & 1.476 & 0.1431 & 699.1 & 293.0 & 1.309 & 4.604 & 445.9 \\
\hline 30 & 396.72 & 1.476 & 0.1458 & 685.8 & 293.0 & 1.309 & 4.517 & 445.9 \\
\hline 31 & 410.40 & 1.476 & 0.1488 & 672.2 & 293.0 & 1.309 & 4.427 & 445.9 \\
\hline 32 & 424.08 & 1.476 & 0.1519 & 658.4 & 293.0 & 1.309 & 4.336 & 445.9 \\
\hline 33 & 437.76 & 1.476 & 0.1553 & 644.2 & 293.0 & 1.309 & 4.242 & 445.9 \\
\hline 34 & 451.44 & 1.476 & 0.1589 & 629.6 & 293.0 & 1.309 & 4.146 & 445.9 \\
\hline 35 & 465.12 & 1.476 & 0.1627 & 614.7 & 293.0 & 1.309 & 4.048 & 445.9 \\
\hline 36 & 478.80 & 1.476 & 0.1669 & 599.4 & 293.0 & 1.309 & 3.947 & 445.9 \\
\hline 37 & 492.48 & 1.476 & 0.1714 & 583.7 & 293.0 & 1.309 & 3.844 & 445.9 \\
\hline 38 & 506.16 & 1.476 & 0.1763 & 567.5 & 293.0 . & 1.309 & 3.737 & 445.9 \\
\hline 39 & 34 & 1.476 & 0.1816 & 550.7 & 293.0 & 1.309 & 3.627 & 445.9 \\
\hline
\end{tabular}


SNF-5331, Rev. 0

Verify 13.aro Natural Gas flow example 6.5 from Saad pp. 270

\begin{tabular}{|c|c|r|r|r|r|r|r|r|}
\hline Point & $x$ & $\begin{array}{c}\text { Mass } \\
\text { Flow } \\
(\mathrm{kg} / \mathrm{sec})\end{array}$ & $\begin{array}{c}\text { Mach } \\
\text { (meters) }\end{array}$ & $\begin{array}{c}\mathrm{P} \\
\text { Static } \\
(\mathrm{kPa})\end{array}$ & $\begin{array}{c}\mathrm{T} \\
\text { Static } \\
\text { (deg. K) }\end{array}$ & Gamma & $\begin{array}{c}\text { Rho } \\
\text { Static } \\
(\mathrm{kg} / \mathrm{m} 3)\end{array}$ & $\begin{array}{c}\text { Vol. } \\
\text { Sonic } \\
\text { (meters/sec) }\end{array}$ \\
\hline 40 & 533.52 & 1.476 & 0.1875 & 533.4 & 293.0 & 1.309 & 3.513 & 445.9 \\
\hline 41 & 547.20 & 1.476 & 0.1941 & 515.5 & 293.0 & 1.309 & 3.395 & 445.9 \\
\hline 42 & 560.88 & 1.476 & 0.2013 & 496.8 & 293.0 & 1.309 & 3.272 & 445.9 \\
\hline 43 & 574.56 & 1.476 & 0.2096 & 477.3 & 293.0 & 1.309 & 3.143 & 445.9 \\
\hline 44 & 588.24 & 1.476 & 0.2189 & 456.9 & 293.0 & 1.309 & 3.009 & 445.9 \\
\hline 45 & 600.95 & 1.476 & 0.2289 & 436.9 & 293.0 & 1.309 & 2.877 & 445.9 \\
\hline 46 & 612.03 & 1.476 & 0.2389 & 418.6 & 293.0 & 1.309 & 2.757 & 445.9 \\
\hline 47 & 621.73 & 1.476 & 0.2489 & 401.8 & 293.0 & 1.309 & 2.646 & 445.9 \\
\hline 48 & 630.27 & 1.476 & 0.2589 & 386.3 & 293.0 & 1.309 & 2.544 & 445.9 \\
\hline 49 & 637.83 & 1.476 & 0.2689 & 371.9 & 293.0 & 1.309 & 2.449 & 445.9 \\
\hline 50 & 644.53 & 1.476 & 0.2789 & 358.6 & 293.0 & 1.309 & 2.362 & 445.9 \\
\hline 51 & 650.50 & 1.476 & 0.2889 & 346.2 & 293.0 & 1.309 & 2.280 & 445.9 \\
\hline 52 & 655.83 & 1.476 & 0.2989 & 334.6 & 293.0 & 1.309 & 2.204 & 445.9 \\
\hline 53 & 660.61 & 1.476 & 0.3069 & 323.8 & 293.0 & 1.309 & 2.132 & 445.9 \\
\hline 54 & 664.91 & 1.476 & 0.3189 & 313.6 & 293.0 & 1.309 & 2.065 & 445.9 \\
\hline 55 & 668.79 & 1.476 & 0.3289 & 304.1 & 293.0 & 1.309 & 2.003 & 445.9 \\
\hline 56 & 672.29 & 1.476 & 0.3389 & 295.1 & 293.0 & 1.309 & 1.944 & 445.9 \\
\hline 57 & 675.47 & 1.476 & 0.3489 & 286.7 & 293.0 & 1.309 & 1.888 & 445.9 \\
\hline 58 & 678.35 & 1.476 & 0.3589 & 278.7 & 293.0 & 1.309 & 1.835 & 445.9 \\
\hline 59 & 680.97 & 1.476 & 0.3689 & 271.1 & 293.0 & 1.309 & 1.785 & 445.9 \\
\hline 60 & 683.35 & 1.476 & 0.3789 & 264.0 & 293.0 & 1.309 & 1.738 & 445.9 \\
\hline 61 & 684.00 & 1.476 & 0.3818 & 262.0 & 293.0 & 1.309 & 1.725 & 445.9 \\
\hline & & & & & & & & \\
\hline
\end{tabular}

Internal Pipe Reaults, Pipe 2, Name: L1-Choke

\begin{tabular}{|c|c|c|c|c|c|c|c|c|}
\hline Point & $\begin{array}{c}x \\
\text { (meters) }\end{array}$ & $\begin{array}{c}\text { Mass } \\
\text { Flow } \\
\text { (kg/sec) }\end{array}$ & $\begin{array}{c}\text { Mach } \\
*\end{array}$ & $\begin{array}{c}P \\
\text { Static } \\
(\mathrm{kPa})\end{array}$ & $\begin{array}{c}\mathrm{T} \\
\text { Static } \\
\text { (deg. K) }\end{array}$ & Gamma & $\begin{array}{c}\text { Rho } \\
\text { Static } \\
(\mathrm{ko} / \mathrm{m} 3)\end{array}$ & $\begin{array}{c}\text { Vel. } \\
\text { Sonic } \\
\text { (meters/sec) }\end{array}$ \\
\hline 1 & 0.00 & 1.476 & 0.1000 & 1000.0 & 293.0 & 1.309 & 6.5855 & 445.9 \\
\hline 2 & 14.20 & 1.476 & 0.1010 & 990.5 & 293.0 & 1.309 & 6.5231 & 445.9 \\
\hline 3 & 28.39 & 1.476 & 0.1020 & 981.0 & 293.0 & 1.309 & 6.4602 & 445.9 \\
\hline 4 & 42.59 & 1.476 & 0.1030 & 971.3 & 293.0 & 1.309 & 6.3966 & 445.9 \\
\hline 5 & 56.79 & 1.476 & 0.1040 & 961.6 & 293.0 & 1.309 & 6.3323 & 445.9 \\
\hline 6 & 70.98 & 1.476 & 0.1051 & 951.7 & 293.0 & 1.309 & 6.2673 & 445.9 \\
\hline 7 & 85.18 & 1.476 & 0.1062 & 941.7 & 293.0 & 1.309 & 6.2017 & 445.9 \\
\hline 8 & 99.37 & 1.476 & 0.1074 & 931.6 & 293.0 & 1.309 & 6.1353 & 445.9 \\
\hline 9 & 113.57 & 1.476 & 0.1086 & 921.4 & 293.0 & 1.309 & 6.0682 & 445.9 \\
\hline 10 & 127.77 & 1.476 & 0.1098 & 911.1 & 293.0 & 1.309 & 6.0003 & 445.9 \\
\hline 11 & 141.96 & 1.476 & 0.1111 & 900.7 & 293.0 & 1.309 & 5.9316 & 445.9 \\
\hline 12 & 156.16 & 1.476 & 0.1124 & 890.2 & 293.0 & 1.309 & 5.8621 & 445.9 \\
\hline 13 & 170.36 & 1.476 & 0.1137 & 879.5 & 293.0 & 1.309 & 5.7917 & 445.9 \\
\hline 14 & 184.55 & 1.476 & 0.1152 & 868.6 & 293.0 & 1.309 & 5.7204 & 445.9 \\
\hline 15 & 198.75 & 1.476 & 0.1166 & 857.7 & 293.0 & 1.309 & 5.6482 & 445.9 \\
\hline 16 & 212.94 & 1.476 & 0.1182 & 846.6 & 293.0 & 1.309 & 5.5750 & 445.9 \\
\hline 17 & 227.14 & 1.476 & 0.1198 & 835.3 & 293.0 & 1.309 & 5.5008 & 445.9 \\
\hline 18 & 241.34 & 1.476 & 0.1214 & 823.9 & 293.0 & 1.309 & 5.4255 & 445.9 \\
\hline 19 & 255.53 & 1.476 & 0.1231 & 812.3 & 293.0 & 1.309 & 5.3492 & 445.9 \\
\hline 20 & 269.73 & 1.476 & 0.1250 & 800.5 & 293.0 & 1.309 & 5.2717 & 445.9 \\
\hline 21 & 283.93 & 1.476 & 0.1269 & 788.5 & 293.0 & 1.309 & 5.1930 & 445.9 \\
\hline 22 & 298.12 & 1.476 & 0.1288 & 776.4 & 293.0 & 1.309 & 5.1130 & 445.9 \\
\hline 23 & 312.32 & 1.476 & 0.1309 & 764.1 & 293.0 & 1.309 & 5.0317 & 445.9 \\
\hline 24 & 326.51 & 1.476 & 0.1331 & 751.5 & 293.0 & 1.309 & 4.9490 & 445.9 \\
\hline 25 & 340.71 & 1.476 & 0.1354 & 738.7 & 293.0 & 1.309 & 4.8648 & 445.9 \\
\hline
\end{tabular}


SNF-5331, Rev. 0

AFT Arrow 2.0 Output

Numatec Hanford Corporation

(4)

$12 / 7 / 99$

Verify13.aro Natural Gas flow example 6.5 from Saad pp. 270

\begin{tabular}{|c|c|c|c|c|c|c|c|c|}
\hline Point & $\begin{array}{c}x \\
\text { (meters) }\end{array}$ & $\begin{array}{c}\text { Mass } \\
\text { Flow } \\
(\mathrm{kg} / \mathrm{sec})\end{array}$ & $\begin{array}{c}\text { Mach } \\
*\end{array}$ & $\begin{array}{c}P \\
\text { Static } \\
(\mathbf{k P a}) \\
\end{array}$ & $\begin{array}{c}T \\
\text { Static } \\
\text { (deg. } K)\end{array}$ & Gamma & $\begin{array}{c}\text { Rho } \\
\text { Static } \\
(\mathrm{kg} / \mathrm{m} 3)\end{array}$ & $\begin{array}{c}\text { Vel. } \\
\text { Sonic } \\
\text { (meters/sec) }\end{array}$ \\
\hline 26 & 354.91 & $1: 476$ & 0.1378 & 725.7 & 293.0 & 1.309 & 4.7790 & 445.9 \\
\hline 27 & 369.10 & 1.476 & 0.1404 & 712.4 & 293.0 & 1.309 & 4.6916 & 445.9 \\
\hline 28 & 383.30 & 1.476 & 0.1431 & 698.9 & 293.0 & 1.309 & 4.6025 & 445.9 \\
\hline 29 & 397.50 & 1.476 & 0.1460 & 685.1 & 293.0 & 1.309 & 4.5115 & 445.9 \\
\hline 30 & 411.69 & 1.476 & 0.1491 & 670.9 & 293.0 & 1.309 & 4.4185 & 445.9 \\
\hline 31 & 425.89 & 1.476 & 0.1524 & 656.5 & 293.0 & 1.309 & 4.3234 & 445.9 \\
\hline 32 & 440.08 & 1.476 & 0.1559 & 641.7 & 293.0 & 1.309 & 4.2260 & 445.9 \\
\hline 33 & 454.28 & 1.476 & 0.1596 & 626.6 & 293.0 & 99 & 4.1262 & 445.9 \\
\hline 34 & 468.48 & 1.476 & 0.1637 & 611.0 & 293.0 & 1.309 & 37 & 445.9 \\
\hline 35 & 482.67 & 1.476 & 0.1681 & 595.0 & 293.0 & 1.309 & 3.9183 & 445.9 \\
\hline 36 & 496.87 & 1.476 & 0.1729 & 578.5 & 293.0 & 1.309 & 3.8098 & 445.9 \\
\hline 37 & 511.07 & 1.476 & 0.1781 & 561.5 & 293.0 & 1.309 & 3.6978 & 445.9 \\
\hline 38 & 525.26 & 1.476 & 0.1839 & 543.9 & 293.0 & 1.309 & 3.5820 & 445.9 \\
\hline 39 & 539.46 & 1.476 & 0.1903 & 525.7 & 293.0 & 1.309 & 3.4619 & 445.9 \\
\hline 40 & 553.65 & 1.476 & 0.1974 & 506.7 & 293.0 & 1.309 & 3.3371 & 445.9 \\
\hline 41 & 567.85 & 1.476 & 0.2054 & 487.0 & 293.0 & 1.309 & 3.2069 & 445.9 \\
\hline 42 & 582.05 & 1.476 & 0.2145 & 466.2 & 293.0 & 1.309 & 3.0704 & 445.9 \\
\hline 43 & 595.57 & 1.476 & 0.2245 & 445.5 & 293.0 & 1.309 & 2.9336 & 445.9 \\
\hline 44 & 607.33 & 1.476 & 0.2345 & 426.5 & 293.0 & 1.309 & 2.8086 & 445.9 \\
\hline 45 & 617.61 & 1.476 & 0.2445 & 409.0 & 293.0 & 1.309 & 2.6937 & 445.9 \\
\hline 46 & 626.64 & 1.476 & 0.2545 & 393.0 & 293.0 & 1.309 & 2.5879 & 445.9 \\
\hline 47 & 634.61 & 1.476 & 0.2645 & 378.1 & 293.0 & 1.309 & 2.4901 & 445.9 \\
\hline 48 & 641.67 & 1.476 & 0.2745 & 364.3 & 293.0 & 1.309 & 2.3994 & 445.9 \\
\hline 49 & 647.95 & 1.476 & 0.2845 & 351.5 & 293.0 & 309 & 2.3150 & 445.9 \\
\hline 50 & 653.55 & 1.476 & 0.2945 & 339.6 & 293.0 & 1.309 & 2.2365 & 445.9 \\
\hline 51 & 658.57 & 1.476 & 0.3045 & 328.5 & 293.0 & 1.309 & 2.1630 & 445.9 \\
\hline 52 & 663.07 & 1.476 & 0.3145 & 318.0 & 293.0 & 1.309 & 2.0942 & 445.9 \\
\hline 53 & 667.13 & 1.476 & 0.3245 & 308.2 & 293.0 & 1.309 & 2.0297 & 445.9 \\
\hline 54 & 670.79 & 1.476 & 0.3345 & 299.0 & 293.0 & 1.309 & 1.9690 & 445.9 \\
\hline 55 & 674.10 & 1.476 & 0.3445 & 290.3 & 293.0 & 1.309 & 1.9119 & 445.9 \\
\hline 56 & 677.11 & 1.476 & 0.3545 & 282.1 & 293.0 & 1.309 & 1.8580 & 445.9 \\
\hline 57 & 679.84 & 1.476 & 0.3645 & 274.4 & 293.0 & 1.309 & 1.8070 & 445.9 \\
\hline 58 & 682.32 & 1.476 & 0.3745 & 267.1 & 293.0 & 1.309 & 1.7588 & 445.9 \\
\hline 59 & 684.59 & 1.476 & 0.3845 & 260.1 & 293.0 & 1.309 & 1.7130 & 445.9 \\
\hline 60 & 686.66 & 1.476 & 0.3945 & 253.5 & 293.0 & 1.309 & 1.6696 & 445.9 \\
\hline 61 & 688.56 & 1.476 & 0.4045 & 247.3 & 293.0 & 1,309 & 1.6283 & 445.9 \\
\hline 62 & 690.29 & 1.476 & 0.4145 & 241.3 & 293.0 & 1.309 & 1.5891 & 445.9 \\
\hline 63 & 691.88 & 1.476 & 0.4245 & 235.6 & 293.0 & 1.309 & 1.5516 & 445.9 \\
\hline 64 & 693.34 & 1.476 & 0.4345 & 230.2 & 293.0 & 1.309 & 1.5159 & 445.9 \\
\hline 65 & 694.69 & 1.476 & 0.4445 & 225.0 & 293.0 & 1.309 & 1.4818 & 445.9 \\
\hline 66 & 695.93 & 1.476 & 0.4545 & 220.1 & 293.0 & 1.309 & 1.4492 & 445.9 \\
\hline 67 & 697.06 & 1.476 & 0.4645 & 215.3 & 293.0 & 1.309 & 1.4180 & 445.9 \\
\hline 68 & 698.11 & 1.476 & 0.4745 & 210.8 & 293.0 & 1.309 & 1.3881 & 445.9 \\
\hline 69 & 699.08 & 1.476 & 0.4845 & 206.4 & 293.0 & 1.309 & 1.3595 & 445.9 \\
\hline 70 & 699.98 & 1.476 & 0.4945 & 202.3 & 293.0 & 1.309 & 1.3320 & 445.9 \\
\hline 71 & 700.80 & 1.476 & 0.5045 & 198.3 & 293.0 & 1.309 & 1.3056 & 445.9 \\
\hline 72 & 701.56 & 1.476 & 0.5145 & 194.4 & 293.0 & 1.309 & 1.2802 & 445.9 \\
\hline 73 & 702.27 & 1.476 & 0.5245 & 190.7 & 293.0 & 1.309 & 1.2558 & 445.9 \\
\hline 74 & 702.92 & 1.476 & 0.5345 & 187.1 & 293.0 & 1.309 & 1.2323 & 445.9 \\
\hline 75 & 703.52 & 1.476 & 0.5445 & 183.7 & 293.0 & 1.309 & 1.2097 & 445.9 \\
\hline 76 & 704.08 & 1.476 & 0.5545 & 180.4 & 293.0 & 1.309 & 1.1879 & 445.9 \\
\hline$\pi$ & 704.59 & 1.476 & 0.5645 & 177.2 & 293.0 & 1.309 & 1.1668 & 445.9 \\
\hline 78 & 705.07 & 1.476 & 0.5745 & 174.1 & 293.0 & 1.309 & 1.1465 & 445.9 \\
\hline
\end{tabular}


SNF-5331, Rev. 0

AFT Arrow 2.0 Output

(5)

$12 \pi / 99$

Numatec Hanford Corporation

Verify13.aro Natural Gas flow example 6.5 from Saad pp. 270

\begin{tabular}{|c|c|c|c|c|c|c|c|c|}
\hline Point & $\begin{array}{c}x \\
\text { (meters) }\end{array}$ & $\begin{array}{c}\text { Mass } \\
\text { Flow } \\
(\mathrm{kg} / \mathrm{sec})\end{array}$ & $\begin{array}{c}\text { Mach } \\
\#\end{array}$ & $\begin{array}{c}P \\
\text { Static } \\
(\mathrm{kPa})\end{array}$ & $\begin{array}{c}\mathrm{T} \\
\text { Static } \\
(\operatorname{deg} . \mathrm{K})\end{array}$ & Gamma & $\begin{array}{c}\text { Rho } \\
\text { Static } \\
(\mathrm{kg} / \mathrm{m} 3)\end{array}$ & $\begin{array}{c}\text { Vel. } \\
\text { Sonic } \\
\text { (meters/sec) }\end{array}$ \\
\hline 79 & 705.51 & 1.476 & 0.5845 & 171.1 & 293.0 & 1.309 & 1.1269 & 445.9 \\
\hline 80 & 705.91 & 1,476 & 0.5945 & 168.2 & 293.0 & 1.309 & 1.1080 & 445.9 \\
\hline 81 & 706.29 & 1.476 & 0.6045 & 165.5 & 293.0 & 1.309 & 1.0896 & 445.9 \\
\hline 82 & 706.63 & 1.476 & 0.6145 & 162.8 & 293.0 & 1.309 & 1.0719 & 445.9 \\
\hline 83 & 706.95 & 1.476 & 0.6245 & 160.2 & 293.0 & 1.309 & 1.0547 & 445.9 \\
\hline 84 & 707.25 & 1.476 & 0.6345 & 157.6 & 293.0 & 1.309 & 1.0381 & 445.9 \\
\hline 85 & 707.52 & 1.476 & 0.6445 & 155.2 & 293.0 & 309 & 1.0220 & 445.9 \\
\hline 86 & 707.77 & 1.476 & 0.6545 & 152.8 & 293.0 & 1.309 & 1.0064 & 445.9 \\
\hline 87 & 708.00 & 1.476 & 0.6645 & 150.5 & 293.0 & 1.309 & .9913 & 445.9 \\
\hline 88 & 708.21 & 1.476 & 0.6745 & 148.3 & 293.0 & 1.309 & 0.9766 & 445.9 \\
\hline 89 & 708.40 & 1.476 & 0.6845 & 146.1 & 293.0 & 1.309 & 0.9623 & 445.9 \\
\hline 90 & 708.58 & 1.476 & 0.6945 & 144.0 & 293.0 & 1.309 & 0.9484 & 445.9 \\
\hline 91 & 708.74 & 1.476 & 0.7045 & 142.0 & 293.0 & 1.309 & 0.9350 & 445.9 \\
\hline 92 & 708.88 & 1.476 & 0.7145 & 140.0 & 293.0 & 1.309 & 0.9219 & 445.9 \\
\hline 93 & 709.02 & 1.476 & 0.7245 & 138.1 & 293.0 & & 0.9092 & 445.9 \\
\hline 94 & 709.13 & 1.476 & 0.7345 & 136.2 & 293.0 & 1.309 & 0.8968 & 445.9 \\
\hline 95 & 709.24 & 1.476 & 0.7445 & 134.3 & 293.0 & 1.309 & 0.8847 & 445.9 \\
\hline 96 & 709.34 & 1.476 & 0.7545 & 132.6 & 3.0 & & 730 & 445.9 \\
\hline 97 & 709.42 & 1.476 & 0.7645 & 130.8 & 293.0 & 1.309 & 0.8616 & 445.9 \\
\hline 98 & 709.50 & 1.476 & 0.7745 & 129.1 & 293.0 & 1.309 & 0.8505 & 445.9 \\
\hline 99 & 709.56 & 1.476 & 0.7845 & 127.5 & 293.0 & 1.309 & 0.8396 & 445.9 \\
\hline 100 & 709.62 & 1.476 & 0.7945 & 125.9 & 293.0 & 1.309 & 0.8291 & 445.9 \\
\hline 101 & 709.67 & 1.476 & 0.8045 & 124.3 & 293.0 & 1.309 & 0.8188 & 445.9 \\
\hline 102 & 709.71 & 1.476 & 0.8145 & 122.8 & 293.0 & 1.309 & 0.8087 & 445.9 \\
\hline 103 & 709.74 & 1.476 & 0.8245 & 121.3 & 293.0 & 1.309 & 0.7989 & 445.9 \\
\hline 104 & 709.77 & 1.476 & 0.8345 & 119.9 & 293.0 & 1.309 & 0.7893 & 445.9 \\
\hline 105 & 709.79 & 1.476 & 0.8445 & 118.4 & 293.0 & 1.309 & 0.7800 & 445.9 \\
\hline 106 & 709.80 & 1.476 & 0.8545 & 117.1 & 293.0 & 1.309 & 0.7709 & 445.9 \\
\hline 107 & 709.81 & 1.476 & 0.8645 & 115.7 & 293.0 & 1.309 & 0.7619 & 445.9 \\
\hline 108 & 709.81 & 1.476 & 0.8704 & 114.9 & 293.0 & 1.309 & 0.7568 & 445.9 \\
\hline
\end{tabular}

All Junction Table

\begin{tabular}{|c|r|r|}
\hline Jct & $\begin{array}{c}\text { P Static } \\
\text { In } \\
(\mathrm{kPa})\end{array}$ & $\begin{array}{r}\text { P Static } \\
\text { Out } \\
(\mathrm{kPa})\end{array}$ \\
\hline 1 & 1000.0 & 1000.0 \\
\hline 2 & 262.0 & 262.0 \\
\hline 3 & 1000.0 & 1000.0 \\
\hline 4 & 114.9 & 114.9 \\
\hline
\end{tabular}


TITLE: VERIFY14.ARO

REFERENCE: Robert H. Perry and Don W. Green Editors, Author James. N. Tilton, Perry's Chemical Engineer's Handbook, Seventh Edition, Page 6-25, example 8

GAS: Air

ASSUMPTIONS: 1) Adiabatic, 2) Perfect gas

RESULTS:

\begin{tabular}{|l|r|r|}
\hline Parameter & Tilton & AFT Arrow 2.0 \\
\hline Mass flow rate $(\mathrm{kg} / \mathrm{s})$ & 2.7 & 2.716 \\
\hline
\end{tabular}

\section{DISCUSSION:}

The objective is to solve for the choked flow rate. The results agree closely.

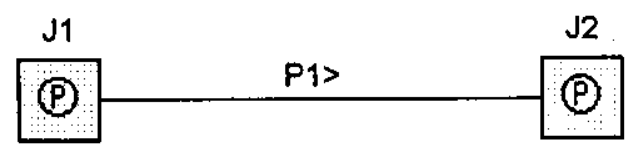

Copyright 1999 Applied Flow Technology Corp. All rights reserved. 
Robert H. Perry and Don W. Green Editors, Author James. N. Tilton

Perry's Chemical Engineer's Handbook, Seventh Edition

Page 6-25, example 8

Air flow - Adiabatic

See Verify 14.doc MS Word file for comparison with published results

Title: Verify14.aro - Air flow Example 8 from Perry's pp. 6-25

Number Of Pipes $=1$

Number Of Junctions $=2$

Length March Solution Method with Mach Number Limits

Segments Per Pipe= 10

Mach Number Increment= 01

Pressure Tolerance $=.0001$ relative change

Mass Flow Rate Tolerance $=.0001$ relative change

Enthalpy Tolerance $=.0001$ relative change

Flow Relaxation $=.5$

Pressure Relaxation $=.5$

Fluid Database: AFT Standard

Fluid= Air

Max Fluid Temperature Data $=1000$ deg. $K$

Min Fluid Temperature Data $=200$ deg. $K$

Molecular Weight $=28.97 \mathrm{amu}$

Gas Constant $=0.2868 \mathrm{~kJ} / \mathrm{kg}-\mathrm{K}$

Critical Pressure $=37.25 \mathrm{~atm}$

Critical Temperature $=132.41$ deg. $K$

Acentric Factor= .021

Equation of State= Ideal Gas

Enthalpy Model= Reference

Specific Heat Ratio Accuracy= Standard

Atmospheric Pressure $=1 \mathrm{~atm}$

Gravitational Acceleration $=1$ g's

Standard Pressure $=0.1 \mathrm{MPa}$

Standard Temperature $=293$ deg. $K$

Turbulent Flow Above Reynoids Number $=4000$

Laminar Flow Below Reynolds Number= 2300

Specific Heat Ratio Accuracy = Standard

Atmospheric Pressure $=1 \mathrm{~atm}$

Gravitational Acceleration $=1 \mathrm{~g}$

Standard Pressure $=0.1 \mathrm{MPa}$

Standard Temperature $=293 \mathrm{deg} . \mathrm{K}$

Turbulent Flow Above Reynolds Numberz 4000

Laminar Flow Below Reynolds Number $=\mathbf{2 3 0 0}$

Pipe Input Table

\begin{tabular}{|c|r|r|r|r|r|r|r|r|r|r|}
\hline Pipe & Name & $\begin{array}{c}\text { Pipe } \\
\text { Defined }\end{array}$ & Length & $\begin{array}{c}\text { Length } \\
\text { Units }\end{array}$ & $\begin{array}{c}\text { Hydraulic } \\
\text { Diameter }\end{array}$ & $\begin{array}{c}\text { Hydraulic } \\
\text { Diam. Units }\end{array}$ & Roughness & $\begin{array}{c}\text { Roughness } \\
\text { Units }\end{array}$ & Losses (K) & Initial Flow \\
\hline 1 & Pipe & Yes & 10 & meters & 2.067 & inches & 0.0188 & Explicit f & & 2 \\
\hline
\end{tabular}

\begin{tabular}{|c|c|c|c|c|c|c|c|}
\hline Pipe & $\begin{array}{c}\text { Initial Flow } \\
\text { Units }\end{array}$ & $\begin{array}{c}\text { Junctions } \\
(\text { Up, Down })\end{array}$ & Geometry & Material & Size & Type & $\begin{array}{c}\text { Special } \\
\text { Condition }\end{array}$ \\
\hline 1 & & 1,2 & Cylindrical Pipe & Steel & 2 inch & schedule 40 & None \\
\hline
\end{tabular}

\section{Assigned Pressure Table}


SNF-5331, Rev. 0

AFT Arrow 2.0 Input

Numatec Hanford Corporation

Verify14.aro - Air flow Example 8 from Perry's pp. 6-25

\begin{tabular}{|c|c|r|r|r|r|r|r|r|}
\hline Assigned Pressure & Name & $\begin{array}{c}\text { Object } \\
\text { Defined }\end{array}$ & $\begin{array}{c}\text { Database } \\
\text { Source }\end{array}$ & $\begin{array}{c}\text { Special } \\
\text { Condition }\end{array}$ & Fluid & Pressure & $\begin{array}{c}\text { Pressure } \\
\text { Units }\end{array}$ & Temperature \\
\hline 1 & Assigned Pressure & Yes & & N/A & Air & 1 & $\mathrm{MPa}(\mathrm{g})$ & 20 \\
\hline 2 & Assigned Pressure & Yes & & N/A & Air & 1 & atm & 20 \\
\hline
\end{tabular}

\begin{tabular}{|c|r|r|}
\hline Assigned Pressure & $\begin{array}{c}\text { Temperature } \\
\text { Units }\end{array}$ & $\begin{array}{c}\text { Loss } \\
\text { Factor }\end{array}$ \\
\hline 1 & deg. C & 0 \\
\hline 2 & deg. C & 0 \\
\hline
\end{tabular}


Robert H. Perry and Don W. Green Editors, Author James. N. Tilton

Perry's Chemical Engineer's Handbook, Seventh Edition

Page 6-25, example 8

Air flow - Adiabatic

See Verify 14.doc MS Word fle for comparison with published results

Title: Verify14.aro - Air flow Example 8 from Perry's pp. 6-25

Analysis run on: $127 / 99$ 12:13:43 PM

Input File: C:MFT ProductsLAFT AmowlVerificationlverify14.aro

Execution Time= 1.65 seconds

Total Number Of Pressure Iterations $=0$

Total Number Of Flow lterations= 2

Total Number Of Enthalpy lterations= 2

Number Of Pipes= 1

Number of Junctions= 2

Length March Solution Method with Mach Number Limits

Segments Per Pipe $=10$

Mach Number increment $=.01$

Pressure Tolerance $=0001$ relative change

Mass Flow Rate Tolerence= .0001 relative change

Enthalpy Tolerance= .0001 relative change

Flow Relaxation $=.5$

Pressure Relaxation $=.5$

Fluid Database: AFT Standard

Fluid= Air

Max Fluid Temperature Data $=1000 \mathrm{deg} . \mathrm{K}$

Min Fluid Temperature Data $=200 \mathrm{deg} . \mathrm{K}$

Molecular Weight= $28.97 \mathrm{amu}$

Gas Constant $=0.2868 \mathrm{~kJ} / \mathrm{kg}-\mathrm{K}$

Critical Pressure $=37.25$ atm

Critical Temperature $=132.41 \mathrm{deg} . \mathrm{K}$

Acentric Factor= 021

Equation of State= Ideal Gas

Enthalpy Model= Reference

Specific Heat Ratio Accuracy= Standard

Atmospheric Pressure= $1 \mathrm{~atm}$

Gravitational Acceleration $=1 \mathrm{~g} / 8$

Standard Pressure= $0.1 \mathrm{MPa}$

Standard Temperature $=293 \mathrm{deg} . \mathrm{K}$

Turbulent Flow Above Reynolds Number $=4000$

Laminar Flow Below Reynolds Numberm $\mathbf{2 3 0 0}$

Specific Heat Ratio Accuracy= Standard

Atmospheric Pressure= $1 \mathrm{~atm}$

Gravitational Acceleration $=1 \mathrm{~g}$

Standard Preasure $=0.1 \mathrm{MPa}$

Standard Temperature $=293$ deg. K

Turbulent Flow Above Reynolds Number $=\mathbf{4 0 0 0}$

Laminar Flow Below Reynolds Number $=2300$

Overall Delta Pressure $=-1.000 \mathrm{MPa}$

Total Inflow $=2.716 \mathrm{~kg} / \mathrm{sec}$

Total Outflow $=2.716 \mathrm{~kg} / \mathrm{sec}$

Total Energy Inflow= 1230. kW

Total Energy Outflow= 1230. kW

Total Heat Transferred Into System $0.000 \mathrm{~kW}$

Maximum Pressure is 1.037 MPa Junction 1 Inlet

Minimum Pressure is $0.1013 \mathrm{MPa}$ at Junction 2 Outlet

Maximum Static Temperature is $20.00 \mathrm{deg}$. C at Junction 1 Inlet

Minimum Static Temperature is $20.00 \mathrm{deg}$. C at Junction 1 Iniet

***CAUTION*** Junction 2 is downetream of a shock, static properties cannot be calculated at junction.

Sonic Choking Exists at Junction 2 (Assigned Pressure) 
Verify 14.aro - Air flow Example 8 from Perry's pp. 6-25

\begin{tabular}{|c|c|c|c|c|c|c|c|c|c|c|}
\hline Pipe & $\begin{array}{c}\text { Mass } \\
\text { Flow } \\
(\mathrm{kg} / \mathrm{sec})\end{array}$ & $\begin{array}{c}\text { Mach } \\
\text { \# In }\end{array}$ & $\begin{array}{c}\text { Mach } \\
\text { * Out }\end{array}$ & $\begin{array}{c}\text { P Static } \\
\text { In } \\
(\mathrm{KPa})\end{array}$ & $\begin{array}{c}\text { P Static } \\
\text { Out } \\
(\mathrm{KPa})\end{array}$ & $\begin{array}{c}\text { T Static } \\
\text { In } \\
(\mathrm{deg} . \mathrm{K})\end{array}$ & $\begin{array}{c}\text { T Static } \\
\text { Out } \\
(\mathrm{deg} . \mathrm{K})\end{array}$ & $\mathrm{K}$ & fLD & $\begin{array}{c}\text { fU } \\
\mathrm{D}+\mathrm{K}\end{array}$ \\
\hline 1 & 2.716 & 0.2948 & 0.9946 & 1037 & 283.5 & 288.2 & 245.4 & 2.000 & 3.581 & 5.581 \\
\hline
\end{tabular}

\section{All Junction Table}

\begin{tabular}{|c|c|c|}
\hline Jet & $\begin{array}{c}\text { P Static } \\
\text { In } \\
(\mathrm{kPa})\end{array}$ & $\begin{array}{c}\text { P Static } \\
\text { Out } \\
(\mathrm{kPa})\end{array}$ \\
\hline 1 & 1037.2 & 1037.2 \\
\hline 2 & 532.3 & 101.3 \\
\hline
\end{tabular}




\section{TITLE: VERIFY15.ARO}

REFERENCE: William S. Janna, Introduction to Fluid Mechanics, PWS Publishers, Belmont, CA 1983, Pages 317-319, example 8.8

GAS: Air

ASSUMPTIONS: 1) Adiabatic flow, 2) Perfect gas

\section{RESULTS:}

\begin{tabular}{|l|r|r|}
\hline \multicolumn{1}{|c|}{ Parameter } & \multicolumn{1}{|c|}{ Janna } & AFT Arrow 2.0 \\
\hline$M_{2}$ - Mach number at exit & 0.14 & 0.1355 \\
\hline$P_{2}-$ Pressure at exit (psia) & 9.63 & 9.836 \\
\hline$T_{2}-$ Temperature at exit (deg. R) & 528.8 & 528.9 \\
\hline
\end{tabular}

\section{DISCUSSION:}

As specified, inlet conditions are known and outlet conditions need to be determined. With the known inlet conditions, an implied mass flow rate exists. To pose the problem in AFT Arrow terms, a few simple calculations are needed to obtain the mass flow rate. Once obtained, it is applied as a flow demand at the exit.

The problem states that the inlet velocity is $100 \mathrm{ft} / \mathrm{s}, P_{1}=15 \mathrm{psia}, T_{1}=530 \mathrm{R}$. From the ideal gas law, density, and mass flow rate are:

$$
\begin{aligned}
& \rho_{1}=\frac{P_{1}}{R T_{1}}=\frac{15 \mathrm{psia}}{\left(53.34 \frac{\mathrm{ft} \cdot \mathrm{lbf}}{\mathrm{lbm} \cdot \mathrm{R}}\right)(530 \mathrm{R})}=0.0764 \mathrm{lbm} / \mathrm{ft}^{3} \\
& \dot{m}=\rho_{1} V_{1} A=\rho_{1}\left(M_{1} a_{1}\right) A=\left(0.0764 \frac{\mathrm{lbm}}{\mathrm{ft}^{3}}\right)\left(100 \frac{\mathrm{ft}}{\mathrm{s}}\right)\left(\frac{\pi}{4} 2.067^{2} \mathrm{in}^{2}\right)=0.17814 \mathrm{lbm} / \mathrm{s}
\end{aligned}
$$

With this flow rate at the exit, the predictions agree very closely.

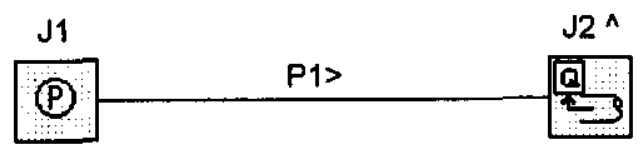

Copyright $\mathcal{C} 1999$ Applied Flow Technology Corp. All rights reserved. 
SNF-5331, Rev. 0

AFT Arrow 2.0 Input

Numatec Hanford Corporation

Verify15.aro - Air flow in a pipe, Example 8.8 from Janna page 317-319

William S. Janna

Introduction to Fluid Mechanics, PWS Publishers, Belmont, CA 1983

Pages 317-319, example 8.8

Air flow - Adiabatic flow, perfect gas assumption

See Verify15.doc MS Word file for comparison with published results

Title: Verify15.aro - Air flow in a pipe, Example 8.8 from Janna page 317-319

Number Of Pipes= 1

Number Of Junctions $=2$

Length March Solution Method with Mach Number Limits

Segments Per Pipe= 2

Mach Number Increment $=.01$

Pressure Tolerance $=.0001$ relative change

Mass Flow Rate Tolerance $=.0001$ relative change

Enthalpy Tolerance $=, 0001$ relative change

Flow Relaxation= (Automatic)

Pressure Relaxation $=$ (Automatic)

Fluid Database: AFT Standard

Fluid= Air

Max Fluid Temperature Data $=1000$ deg. $K$

Min Fluid Temperature Data $\mathbf{2 0 0}$ deg. $\mathrm{K}$

Molecular Weight $=28.97 \mathrm{amu}$

Gas Constant $=0.068549$ Btu/bm-R

Critical Pressure $=37.25 \mathrm{~atm}$

Critical Temperature $=132.41 \mathrm{deg} . \mathrm{K}$

Acentric Factor $=.021$

Equation of State $=$ Ideal Gas

Enthalpy Model= Reference

Specific Heat Ratio Accuracy $=$ High

Atmospheric Pressure $=1 \mathrm{~atm}$

Gravitational Acceleration= $1 \mathrm{~g}$ 's

Standard Pressure $=14.696 \mathrm{psia}$

Standard Temperature $=60$ deg. $F$

Turbulent Flow Above Reynolds Number $=4000$

Laminar Flow Below Reynolds Number $=2300$

Specific Heat Ratio Accuracy= High

Atmospheric Pressure $=1 \mathrm{~atm}$

Gravitational Acceleration $=1 \mathrm{~g}$

Standard Pressure $=14.696 \mathrm{psia}$

Standard Temperature $=60$ deg. $F$

Turbulent Flow Above Reynolds Number $=4000$

Laminar Flow Below Reynolds Number $=\mathbf{2 3 0 0}$

Pipe Input Table

\begin{tabular}{|c|r|r|r|r|r|r|r|r|r|r|}
\hline Pipe & Name & $\begin{array}{c}\text { Pipe } \\
\text { Defined }\end{array}$ & Length & $\begin{array}{c}\text { Length } \\
\text { Units }\end{array}$ & $\begin{array}{r}\text { Hydraulic } \\
\text { Diameter }\end{array}$ & $\begin{array}{c}\text { Hydraulic } \\
\text { Diam. Units }\end{array}$ & Roughness & $\begin{array}{c}\text { Roughness } \\
\text { Units }\end{array}$ & Losses (K) & Initial Flow \\
\hline 1 & Pipe & Yes & 500 & feet & 2.067 & inches & 0.0175 & Explicit $\mathrm{f}$ & 0 & \\
\hline
\end{tabular}

\begin{tabular}{|c|c|r|c|r|c|c|c|}
\hline Pipe & $\begin{array}{c}\text { Initial Flow } \\
\text { Units }\end{array}$ & $\begin{array}{c}\text { Junctions } \\
\text { (Up,Down) }\end{array}$ & Geometry & Material & Size & Type & $\begin{array}{c}\text { Special } \\
\text { Condition }\end{array}$ \\
\hline 1 & & 1,2 & Cylindrical Pipe & PVC & 2 inch & schedule 40 & None \\
\hline
\end{tabular}

\section{Assigned Flow Table}


SNF-5331, Rev. 0

AFT Arrow 2.0 Input

(2)

$12 / 7 / 99$ 12:23 PM

Numatec Hanford Corporation

Verify15.aro - Air flow in a pipe, Example 8.8 from Janna page $317-319$

\begin{tabular}{|c|c|r|r|r|r|r|r|r|r|}
\hline Assigned Flow & Name & $\begin{array}{c}\text { Object } \\
\text { Defined }\end{array}$ & $\begin{array}{c}\text { Database } \\
\text { Source }\end{array}$ & $\begin{array}{c}\text { Special } \\
\text { Condition }\end{array}$ & Fluid & Type & Flow & $\begin{array}{c}\text { Flow } \\
\text { Units }\end{array}$ & Temperature \\
\hline 2 & Assigned Flow & Yes & & None & Air & Outflow & 0.17814 & Ibm/sec & 530 \\
\hline
\end{tabular}

\begin{tabular}{|c|r|r|}
\hline Assigned Flow & $\begin{array}{c}\text { Temperature } \\
\text { Units }\end{array}$ & $\begin{array}{c}\text { Loss } \\
\text { Model }\end{array}$ \\
\hline 2 & deg. $\mathbf{R}$ & 0 \\
\hline
\end{tabular}

Assigned Pressure Table

\begin{tabular}{|c|c|r|r|r|r|r|r|r|}
\hline Assigned Pressure & Name & $\begin{array}{c}\text { Object } \\
\text { Defined }\end{array}$ & $\begin{array}{c}\text { Database } \\
\text { Source }\end{array}$ & $\begin{array}{c}\text { Special } \\
\text { Condition }\end{array}$ & Fluid & Pressure & $\begin{array}{c}\text { Pressure } \\
\text { Units }\end{array}$ & \begin{tabular}{c} 
Temperature \\
\hline 1
\end{tabular} \\
\hline 1 & Assigned Pressure & Yes & & N/A & Air & 15 & psia & 530 \\
\hline
\end{tabular}

\begin{tabular}{|c|c|c|}
\hline Assigned Pressure & $\begin{array}{c}\text { Temperature } \\
\text { Units }\end{array}$ & $\begin{array}{c}\text { Loss } \\
\text { Factor }\end{array}$ \\
\hline 1 & deg. R & 0 \\
\hline
\end{tabular}


William S. Janna

Introduction to Fluid Mechanics, PWS Publishers, Belmont, CA 1983

Pages 317-319, example 8.8

Air flow - Adiabatic flow, perfect gas assumption

See Verify 15.doc MS Word file for comparison with publiahed results

Title: Verify15.aro - Air flow in a pipe, Example 8.8 from Janna page 317-319

Analysis run on: 12/7/99 12:22:35 PM

Input File: C:VAFT ProductsVAT ArowiVerificationlverify15.aro

Execution Time $=0.16$ seconds

Total Number Of Pressure Iterations $=0$

Total Number Of Flow Iterations= 2

Total Number of Enthalpy Iterations $=2$

Number Of Pipes $=1$

Number Of Junctions $=2$

Length March Solution Method with Mach Number Limits

Segments Per Pipe= 2

Mach Number Increment= .01

Pressure Tolerance= .0001 relative change

Mass Flow Rate Tolerance= ,0001 relative change

Enthalpy Tolerance $=.0001$ relative change

Flow Relaxation= (Automatic)

Pressure Relaxation= (Automatic)

Fluid Database: AFT Standard

Fluid= Air

Max Fluid Temperature Data $=1000$ deg. $K$

Min Fluid Temperature Data 200 deg. K

Molecular Woight= $28.97 \mathrm{amu}$

Gas Constant $=0.068549 \mathrm{Btu} / \mathrm{bm}-\mathrm{R}$

Critical Pressure $=37.25 \mathrm{~atm}$

Critical Temperature= $132.41 \mathrm{deg} . K$

Acentric Factor $=.021$

Equation of State= Ideal Gas

Enthalpy Modela Reference

Specific Heat Ratio Accuracy= High

Atmospheric Pressure= 1 atm

Gravitational Acceleration $=1$ g's

Standard Pressure $=14.696$ psia

Standard Temperature $=60 \mathrm{deg} . \mathrm{F}$

Turbulent Flow Above Reynolds Number $=4000$

Laminar Flow Below Reynolds Number $=\mathbf{2 3 0 0}$

Specific Heat Ratio Accuracy= High

Atmospheric Pressure= $1 \mathrm{~atm}$

Gravitational Acceleration $=1 \mathrm{~g}$

Standard Pressure $=14.696$ paia

Standard Temperature $=60 \mathrm{deg} . \mathrm{F}$

Turbulent Flow Above Reynolds Number $=\mathbf{4 0 0 0}$

Laminar Flow Below Reynolds Number= 2300

Overall Delta Pressure $=-5.1205$ peia

Total Inflow= $0.17814 \mathrm{lbm} / \mathrm{sec}$

Total Outiow $=0.17814 \mathrm{lbm} / \mathrm{sec}$

Total Energy Inflow= 34.844 Btw/s

Total Energy Outflow= $34.844 \mathrm{Btu} / \mathrm{s}$

Total Heat Transferred Into System $=0.0000 \mathrm{Btu} / \mathrm{s}$

Maximum Pressure is 15.000 psia at Junction 1 Inlet Minimum Pressure is 9.8360 paia at Junction 2 Inlet

Maximum Static Temperature is $71.152 \mathrm{deg}$. F at Junction 2 Inlet Minimum Static Temperature is $71.152 \mathrm{deg}$. F at Junction 1 Inlet

\section{Pipe Output Table}


Verify15.aro - Air flow in a pipe, Example 8.8 from Janna page 317-319

\begin{tabular}{|c|c|c|c|c|c|c|c|c|c|c|}
\hline Pipe & $\begin{array}{c}\text { Mach } \\
\# \text { In }\end{array}$ & $\begin{array}{c}\text { Mach } \\
\text { \# Out }\end{array}$ & $\begin{array}{c}\text { P Static } \\
\text { In } \\
\text { (psia) }\end{array}$ & $\begin{array}{c}\text { P Static } \\
\text { Out } \\
\text { (psia) }\end{array}$ & $\begin{array}{c}\text { T Static } \\
\text { In } \\
\text { (deg. R) }\end{array}$ & $\begin{array}{c}\text { T Static } \\
\text { Out } \\
\text { (deg. R) }\end{array}$ & $\begin{array}{c}\text { Mass } \\
\text { Flow } \\
\text { (lbm/sec) }\end{array}$ & Vel. In & $\begin{array}{c}\text { Vel. } \\
\text { Out } \\
\text { (feet/sec) }\end{array}$ & $\begin{array}{c}\text { Rho } \\
\text { Static In } \\
\text { (feet/sec) }\end{array}$ \\
\hline 1 & 0.088913 & 0.13545 & 15.000 & 9.8360 & 530.00 & 528.92 & 0.17814 & 100.06 & 152.28 & 0.076402 \\
\hline
\end{tabular}

\section{Alt Junction Table}

\begin{tabular}{|c|c|c|c|c|}
\hline Jct & $\begin{array}{c}\text { P Static } \\
\text { In } \\
\text { (psia) }\end{array}$ & $\begin{array}{c}\text { P Static } \\
\text { Out } \\
\text { (psia) }\end{array}$ & $\begin{array}{c}\text { T Static } \\
\text { In } \\
\text { (deg. F) }\end{array}$ & $\begin{array}{c}\text { T Static } \\
\text { Out } \\
\text { (deg. F) }\end{array}$ \\
\hline 1 & 15.0000 & 15.0000 & 70.330 & 70.330 \\
\hline 2 & 9.8360 & 9.8360 & 69.248 & 69.248 \\
\hline
\end{tabular}


TITLE: VERIFY16.ARO

REFERENCE: Mohinder L. Nayyar, Piping Handbook, Sixth Edition, McGraw-Hill, New York, 1992, Page B.339, B.342, Example B8.2

GAS: Air

ASSUMPTIONS: 1) Adiabatic flow, 2) Perfect gas

RESULTS:

\begin{tabular}{|l|r|r|}
\hline Parameter & Nayyar & AFT Arrow 2.0 \\
\hline$M_{1}-$ Mach number at valve & 0.317 & 0.318 \\
\hline$P_{1}-$ Pressure at valve (psia) & 128.46 & 128.66 \\
\hline
\end{tabular}

\section{DISCUSSION:}

The problem assumes an unusual inlet boundary condition where the flow rate is known and the stagnation temperature. AFT Arrow uses the static temperature at the inlet because it is typically associated with a flow rate. To match the $120 \mathrm{~F}$ stagnation temperature, the inlet static temperature was iterated a few times.

The conditions result in sonic choking at the discharge.

The predictions agree very closely.

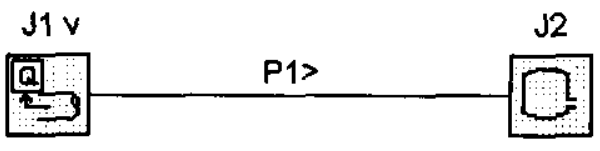

Copyright 11999 Applied Flow Technology Corp. All rights reserved.

* Printed with permission. 
SNF-5331, Rev. 0

AFT Arrow 2.0 Input

Mohinder L. Nayyar

Piping Handbook, Sixth Edition, McGraw-Hill, New York, 1992

Page B.339, B.342, Example B8.2

Air flow - Example assumes air is a perfect gas and adiabatic flow

See Verify16.doc MS Word file for comparison with published results

Title: Verify16.aro - Air flow in a pipe, Example B8.2 from Nayyar Page B.339-342

Number Of Pipes $=1$

Number Of Junctions $=2$

Length March Solution Method with Mach Number Limits

Segments Per Pipe $=10$

Mach Number Increment= 01

Pressure Tolerance $=.0001$ relative change

Mass Flow Rate Tolerance $=.0001$ relative change

Enthalpy Tolerance $=.0001$ relative change

Flow Relaxation $=.5$

Pressure Relaxation $=.5$

Fluid Database: AFT Standard

Fluid= Air

Max Fluid Temperature Data $=1000$ deg. K

Min Fluid Temperature Data $=\mathbf{2 0 0}$ deg. $\mathrm{K}$

Molecular Weight= $28.97 \mathrm{amu}$

Gas Constant $=0.0685486$ Btu/bm-R

Critical Pressure= 37.25 atm

Critical Temperature= $132.41 \mathrm{deg} . \mathrm{K}$

Acentric Factor= 021

Equation of Statex Ideal Gas

Enthalpy Model= Reference

Specific Heat Ratio Accuracy= High

Atmospheric Pressure $=1 \mathrm{~atm}$

Gravitational Acceleration $=1 \mathrm{~g}$ 's

Standard Pressure $=14.696$ paia

Standard Temperature $=60 \mathrm{deg}$. F

Turbulent Flow Above Reynolds Number $=4000$

Laminar Flow Below Reynolds Number= $\mathbf{2 3 0 0}$

Specific Heat Ratio Accuracy= High

Atmospheric Pressure $=1 \mathrm{~atm}$

Gravitational Acceleration $=1 \mathrm{~g}$

Standard Pressure $=14.696 \mathrm{psia}$

Standard Temperature $=60$ deg. F

Turbulent Flow Above Reynolds Number $=4000$

Laminar Flow Below Reynolds Number $=\mathbf{2 3 0 0}$

Pipe input Table

\begin{tabular}{|c|c|c|c|c|c|c|c|c|c|c|}
\hline Pipe & Name & $\begin{array}{c}\text { Pipe } \\
\text { Defined }\end{array}$ & Length & $\begin{array}{l}\text { Length } \\
\text { Units }\end{array}$ & $\begin{array}{l}\text { Hydraulic } \\
\text { Diameter }\end{array}$ & $\begin{array}{l}\text { Hydraulic } \\
\text { Diam. Units }\end{array}$ & Roughness & $\begin{array}{c}\text { Roughness } \\
\text { Units }\end{array}$ & Losses (K) & Initial Flow \\
\hline 1 & Pipe & Yes & 90 & foet & 4.026 & inches & 0.017 & Explicit f & 0 & \\
\hline
\end{tabular}

\begin{tabular}{|c|c|r|c|r|c|c|c|}
\hline Pipe & $\begin{array}{c}\text { Initial Flow } \\
\text { Units }\end{array}$ & $\begin{array}{c}\text { Junctions } \\
(\text { Up,Down })\end{array}$ & Geometry & Material & Size & Type & $\begin{array}{c}\text { Special } \\
\text { Condition }\end{array}$ \\
\hline 1 & & 1,2 & Cylindrical Pipe & Steel & 4 inch & schedule 40 & None \\
\hline
\end{tabular}

Assigned Flow Table 
SNF-5331, Rev. 0

AFT Arrow 2.0 Input

Numatec Hanford Corporation
(2)

12/7/99 12:25 PM

Verify16.aro - Air flow in a pipe, Example B8.2 from Nayyar Page B.339-342

\begin{tabular}{|c|c|c|c|c|c|c|c|c|c|c|}
\hline Assigned Flow & Name & $\begin{array}{c}\text { Object } \\
\text { Defined }\end{array}$ & $\begin{array}{c}\text { Database } \\
\text { Source }\end{array}$ & $\begin{array}{c}\text { Special } \\
\text { Condition }\end{array}$ & Fluid & Type & Flow & $\begin{array}{r}\text { Flow } \\
\text { Units }\end{array}$ & $\begin{array}{r}\text { Temperature } \\
\text { Temperature } \\
\text { Units }\end{array}$ \\
\hline 1 & Assigned Flow & Yes & & None & Air & Inflow & 72000 & $\mathrm{lbm} / \mathrm{hr}$ & 108.75 & deg. F \\
\hline
\end{tabular}

\begin{tabular}{|c|r|}
\hline Assigned Flow & $\begin{array}{c}\text { Loss } \\
\text { Model }\end{array}$ \\
\hline 1 & 0 \\
\hline
\end{tabular}

Tank Table

\begin{tabular}{|c|r|r|r|r|r|r|r|r|r|r|}
\hline Tank & Name & $\begin{array}{c}\text { Object } \\
\text { Defined }\end{array}$ & $\begin{array}{c}\text { Database } \\
\text { Source }\end{array}$ & $\begin{array}{c}\text { Special } \\
\text { Condition }\end{array}$ & Fluid & Pressure & $\begin{array}{c}\text { Pressure } \\
\text { Units }\end{array}$ & Temperature & $\begin{array}{c}\text { Temperature } \\
\text { Units }\end{array}$ & $\begin{array}{c}\text { Balance } \\
\text { Energy }\end{array}$ \\
\hline 2 & Tank & Yes & & N/A & Air & 14.7 & psia & 120 & deg. F & No \\
\hline
\end{tabular}

\begin{tabular}{|c|c|c|c|c|c|c|c|c|c|}
\hline Tank & $\begin{array}{c}\text { Balance } \\
\text { Concentration }\end{array}$ & $\begin{array}{c}\text { (Pipe \#1) } \\
\mathrm{K} \ln , \mathrm{K} \text { Out }\end{array}$ & $\begin{array}{c}\text { (Pipe *2) } \\
\mathrm{K} \ln , \mathrm{K} \text { Out }\end{array}$ & $\begin{array}{c}\text { (Pipe \#3) } \\
\mathrm{K} \ln , \mathrm{K} \text { Out }\end{array}$ & $\begin{array}{c}\text { (Pipe \#4) } \\
\mathrm{K} \ln , \mathrm{K} \text { Out }\end{array}$ & $\begin{array}{c}\text { (Pipe \#5) } \\
\mathrm{K} \ln , \mathrm{K} \text { Out }\end{array}$ & $\begin{array}{c}\text { (Pipe \#6) } \\
\mathrm{K} \ln , \mathrm{K} \text { Out }\end{array}$ & $\begin{array}{c}\text { (Pipe \#7) } \\
\mathrm{K} \text { In, K Out }\end{array}$ & $\begin{array}{c}\text { (Pipe \#8) } \\
\mathrm{K} \ln , \mathrm{K} \text { Out }\end{array}$ \\
\hline 2 & No & $(1) 0,0$ & & & & & & & \\
\hline
\end{tabular}

\begin{tabular}{|c|c|c|}
\hline Tank & $\begin{array}{c}\text { (Pipe \#9) } \\
\text { Kin, K Out }\end{array}$ & $\begin{array}{c}\text { (Pipe \#10) } \\
\text { K In, K Out }\end{array}$ \\
\hline 2 & & \\
\hline
\end{tabular}


Mohinder L. Nayyar

Piping Handbook, Sixth Edition, McGraw-Hill, New York, 1992

Page B.339, B.342, Example B8.2

Air flow - Example assumes air is a perfect gas and adiabatic flow

See Verify16.doc MS Word file for comparison with published results

Title: Verify16.aro - Air flow in a pipe, Example B8.2 from Nayyar Page B.339-342

Analysis run on: 12/7/99 12:25:03 PM

Input File: C:VAFT ProductsVAFT ArrowiVerificationiverify16.aro

Execution Time= 2.91 seconds

Total Number Of Pressure Iterations $=0$

Total Number Of Flow Iterations= 2

Total Number Of Enthalpy Iterations= 2

Number Of Pipes= 1

Number Of Junctions= 2

Length March Solution Method with Mach Number Limite

Segments Per Pipe= 10

Mach Number increment= .01

Pressure Tolerance $=.0001$ relative change

Mass Flow Rate Tolerance $=.0001$ relative change

Enthalpy Tolerance $=.0001$ relative change

Flow Relaxation $=.5$

Pressure Relaxation= .5

Fluid Database: AFT Standard

Fluid= Air

Max Fluid Temperature Data $=1000$ deg. K

Min Fluid Temperature Data 200 deg. K

Molecular Weight= $28.97 \mathrm{amu}$

Gas Constant $=0.0685486 \mathrm{Btu} / \mathrm{hbm}-\mathrm{R}$

Critical Pressure= $37.25 \mathrm{~atm}$

Critical Temperature= $132.41 \mathrm{deg} . \mathrm{K}$

Acentric Factorz .021

Equation of State= Ideal Gas

Enthalpy Model= Reference

Specific Heat Ratio Accuracy $=$ High

Atmospheric Pressure $=1 \mathrm{~atm}$

Gravitational Acceleration=1 g's

Standard Pressure $=14.696$ psia

Standard Temperature= 60 deg. $F$

Turbulent Flow Above Reynolds Number $=4000$

Laminar Flow Below Reynolds Number= 2300

Specific Heat Ratio Accuracy= High

Atmospheric Pressure $=1 \mathrm{~atm}$

Gravitational Acceleration $=1 \mathrm{~g}$

Standard Pressure= $14.696 \mathrm{psia}$

Standard Temperature $=60 \mathrm{deg} . F$

Turbulent Flow Above Reynolds Number $=4000$

Laminar Flow Below Reynolds Number $=2300$

Overall Delta Pressure $=-123.230$ psia

Total Inflow $=20.0000 \mathrm{lbm} / \mathrm{sec}$

Total Outflow= $20.0000 \mathrm{lbm} / \mathrm{sec}$

Total Energy Inflow $=4149.85 \mathrm{Btu} / \mathrm{s}$

Total Energy Outflowe $4149.85 \mathrm{Btu} / \mathrm{s}$

Total Heat Transferred into System $=0.00000 \mathrm{Btw} / \mathrm{s}$

Maximum Pressure is 128.660 psia at Junction 1 Inlet

Minimum Pressure is 14.7000 psia at Junction 2 Outlet

Maximum Static Temperature is 120.000 deg. F at Junction 2 Inlet

Minimum Static Temperalure is 119.986 deg. F at Junction I Inlet

Sonic Choking Exists at Junction 2 (Tank)

Pipe Output Table 
Verify16.aro - Air flow in a pipe, Exemple B8.2 from Nayyar Page B.339-342

\begin{tabular}{|c|c|c|c|c|c|c|c|c|c|c|c|}
\hline Pipe & $\begin{array}{c}\text { Mass } \\
\text { Flow } \\
\text { (lbm/hr) }\end{array}$ & $\begin{array}{c}\text { Vel. In } \\
\text { (feet/sec) }\end{array}$ & $\begin{array}{l}\text { Vel. Out } \\
\text { (feetsec) }\end{array}$ & $\begin{array}{c}\text { Mach } \\
\# \text { In }\end{array}$ & $\begin{array}{l}\text { Mach } \\
* \text { Out }\end{array}$ & $\begin{array}{c}\text { P Static } \\
\text { in } \\
\text { (psia) }\end{array}$ & $\begin{array}{c}\text { P Static } \\
\text { Out } \\
\text { (psia) }\end{array}$ & $\begin{array}{c}\text { T Static } \\
\text { In } \\
\text { (deg. F) }\end{array}$ & $\begin{array}{c}\text { T Static } \\
\text { Out } \\
\text { (deg. F) }\end{array}$ & $\begin{array}{c}\text { T Stag. } \\
\text { In } \\
\text { (deg. F) }\end{array}$ & $\begin{array}{c}\text { T Stag. } \\
\text { Out } \\
\text { (deg. F) }\end{array}$ \\
\hline 1 & $72,000.0$ & 370.246 & 1071.83 & 0.317777 & 0.994971 & 128.660 & 37.9497 & 108.750 & 25.6957 & 119.986 & 120,141 \\
\hline
\end{tabular}

\section{All Junction Table}

\begin{tabular}{|c|c|c|c|c|}
\hline Jet & $\begin{array}{c}\text { P Static } \\
\text { In } \\
\text { (psia) }\end{array}$ & $\begin{array}{c}\text { P Static } \\
\text { Out } \\
\text { (psia) }\end{array}$ & $\begin{array}{c}\text { T Static } \\
\text { In } \\
\text { (deg. F) }\end{array}$ & $\begin{array}{c}\text { T Static } \\
\text { Out } \\
\text { (deg. F) }\end{array}$ \\
\hline 1 & 128.6603 & 128.6603 & 108.750 & 108.750 \\
\hline 2 & 71.2605 & 14.7000 & 120.000 & 120.000 \\
\hline
\end{tabular}


TITLE: VERIFY17.ARO

REFERENCE: Mohinder L. Nayyar, Piping Handbook, Sixth Edition, McGraw-Hill, New York, 1992 , Page B.342, Example B8.3

GAS: Air

ASSUMPTIONS: 1) Adiabatic flow, 2) Perfect gas

RESULTS:

\begin{tabular}{|c|r|r|}
\hline Parameter & \multicolumn{1}{|c|}{ Nayyar } & AFT Arrow 2.0 \\
\hline$M_{1}-$ Mach number at valve & 0.317 & 0.318 \\
\hline$P_{1}-$ Pressure at valve (psia) & 256.93 & 257.32 \\
\hline
\end{tabular}

\section{DISCUSSION:}

The problem assumes an unusual inlet boundary condition where the flow rate is known and the stagnation temperature. AFT Arrow uses the static temperature at the inlet because it is typically associated with a flow rate. To match the $120 \mathrm{~F}$ stagnation temperature, the inlet static temperature was iterated a few times.

The conditions result in sonic choking at the discharge.

The predictions agree very closely.

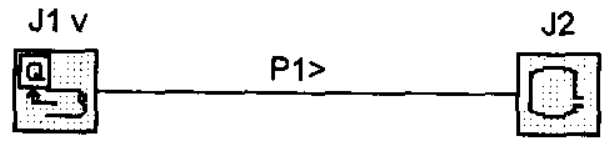

Copyright @1999 Applied Flow Technology Corp. All rights reserved. 
Mohinder L. Nayyar

Piping Handbook, Sixth Edition, MoGraw-Hill, Now York, 1992

Page B.342, Example B8.3

Air flow - Example assumes air is a perfect gas and adiabatic flow

See Verify 17.doc MS Word file for comperison with published results

Tite: Verify 17.aro - Air flow in a pipe, Example B8.3 from Nayyar Page B.342

Number Of Pipes= 1

Number Of Junctions $=2$

Length March Solution Method with Mach Number Limits

Segments Per Pipe= 10

Mach Number Increment= .01

Preasure Tolerances .0001 relative change

Mass Flow Rate Tolerance $=.0001$ relative change

Enthalpy Tolerance $=.0001$ relative change

Concentration Tolerance .0001 relative change

Flow Relaxation $=.5$

Pressure Relaxation $=.5$

Fluid Database: AFT Standard

Fluid= Air

Max Fluid Temperature Data $=1000$ deg. $K$

Min Fluid Temperature Data 200 deg. K

Molecular Weight= 28.97 amu

Gas Constant $=0.0685486 \mathrm{Btu} / \mathrm{bm}-\mathrm{R}$

Critical Pressure= 37.25 atm

Critical Temperature $=132.41 \mathrm{deg} . \mathrm{K}$

Acentric Factor $=.021$

Equation of State= Ideal Gas

Enthalpy Model= Reference

Specific Heat Ratio Accuracy High

Atmospheric Pressure $=1$ atm

Gravitational Acceleration $=1 \mathrm{~g}$ 's

Standard Pressure $=14.696$ psia

Standard Temperature $=60 \mathrm{deg} . F$

Turbulent Flow Above Reynolds Number $=4000$

Laminar Flow Below Reynolds Numberm $\mathbf{2 3 0 0}$

Specific Heat Ratio Accuracy= High

Atmospheric Pressure= 1 atm

Gravitational Acceleration $=1 \mathrm{~g}$

Standard Pressure= 14.696 paia

Standard Temperature $=60 \mathrm{deg} . F$

Turbulent Flow Above Reynolds Number $=4000$

Laminar Flow Below Reynolds Number $=\mathbf{2 3 0 0}$

Pipe Input Table

\begin{tabular}{|c|r|r|r|r|r|r|r|r|r|r|}
\hline Pipe & Name & $\begin{array}{c}\text { Pipe } \\
\text { Defined }\end{array}$ & Length & $\begin{array}{r}\text { Length } \\
\text { Units }\end{array}$ & $\begin{array}{r}\text { Hydraulic } \\
\text { Diameter }\end{array}$ & $\begin{array}{c}\text { Hydraulic } \\
\text { Diam. Units }\end{array}$ & Roughness & $\begin{array}{c}\text { Roughness } \\
\text { Units }\end{array}$ & Losses (K) & Initial Flow \\
\hline 1 & Pipe & Yes & 90 & foet & 4.026 & inches & 0.017 & Explicit $f$ & 0 & 0 \\
\hline
\end{tabular}

\begin{tabular}{|c|c|r|c|r|c|c|c|}
\hline Pipe & $\begin{array}{c}\text { Initial Flow } \\
\text { Units }\end{array}$ & $\begin{array}{c}\text { Junctions } \\
\text { (Up,Down) }\end{array}$ & Geometry & Material & Size & Type & $\begin{array}{c}\text { Special } \\
\text { Condition }\end{array}$ \\
\hline 1 & & 1,2 & Cylindrical Pipe & Steel & 4 inch & schedule 40 & None \\
\hline
\end{tabular}


SNF-5331, Rov. 0

AFT Arrow 2.0 Input

(2)

$127 / 99$ 12:27 PM

Numatec Hanford Corporation

Verify 17.aro - Air flow in a pipe, Example B8.3 from Nayyar Page B.342

\begin{tabular}{|c|c|r|r|r|r|r|r|r|r|r|}
\hline Assigned Flow & Name & $\begin{array}{c}\text { Object } \\
\text { Defined }\end{array}$ & $\begin{array}{c}\text { Databsase } \\
\text { Source }\end{array}$ & $\begin{array}{c}\text { Special } \\
\text { Condition }\end{array}$ & Fluid & Type & Flow & $\begin{array}{r}\text { Flow } \\
\text { Units }\end{array}$ & $\begin{array}{r}\text { Temperature } \\
\text { Temperature } \\
\text { Units }\end{array}$ \\
\hline 1 & Assigned Flow & Yes & & None & Air & Inflow & 144000 & Ibm/hr & 108.75 & deg. F \\
\hline
\end{tabular}

\begin{tabular}{|c|r|}
\hline Assigned Flow & $\begin{array}{c}\text { Loss } \\
\text { Model }\end{array}$ \\
\hline 1 & 0 \\
\hline
\end{tabular}

Tank Table

\begin{tabular}{|c|r|r|r|r|r|r|r|r|r|c|}
\hline Tank & Name & $\begin{array}{c}\text { Object } \\
\text { Defined }\end{array}$ & $\begin{array}{c}\text { Database } \\
\text { Source }\end{array}$ & $\begin{array}{c}\text { Special } \\
\text { Condition }\end{array}$ & Fluid & Pressure & $\begin{array}{c}\text { Pressure } \\
\text { Units }\end{array}$ & Temperature & $\begin{array}{c}\text { Temperature } \\
\text { Units }\end{array}$ & $\begin{array}{c}\text { Balance } \\
\text { Energy }\end{array}$ \\
\hline 2 & Tank & Yes & & N/A & Air & 14.7 & psia & 120 & deg. F & No \\
\hline
\end{tabular}

\begin{tabular}{|c|c|c|c|c|c|c|c|c|c|}
\hline Tank & $\begin{array}{c}\text { Balance } \\
\text { Concentration }\end{array}$ & $\begin{array}{c}\text { (Pipe } * 1) \\
K \ln , K \text { Out }\end{array}$ & $\begin{array}{c}\text { (Pipe *2) } \\
K \ln , K \text { Out } \\
\end{array}$ & $\begin{array}{c}\text { (Pipe } 13 \text { ) } \\
K \ln , K \text { Out }\end{array}$ & $\begin{array}{l}\text { (Pipe \#4) } \\
\mathrm{K} \ln , \mathrm{K} \text { Out }\end{array}$ & $\begin{array}{c}\text { (Pipe }: 5) \\
K \ln , K \text { Out }\end{array}$ & $\begin{array}{l}\text { (Pipe \#6) } \\
\text { KIn, K Out }\end{array}$ & $\begin{array}{c}\text { (Pipe \#7) } \\
K \ln , K \text { Out }\end{array}$ & $\begin{array}{l}\text { (Pipe \#8) } \\
\mathrm{K} \text { In, K Out }\end{array}$ \\
\hline 2 & No & (1) 0,0 & & & & & & & \\
\hline
\end{tabular}

\begin{tabular}{|c|c|c|}
\hline Tank & $\begin{array}{c}\text { (Pipe *9) } \\
\text { KIn, KOut }\end{array}$ & $\begin{array}{c}\text { (Pipe *10) } \\
\text { KIn, Kout }\end{array}$ \\
\hline 2 & & \\
\hline
\end{tabular}


Mohinder L. Nayyar

Piping Handbook, Sixth Edition, McGraw-Hill, New York, 1992

Page B.342, Example B8.3

Air flow - Example assumes air is a perfect gas and adiabatic flow

See Verify17.doc MS Word file for comparison with publiahed results

Title: Verify17.aro - Air flow in a pipe, Example B8.3 from Nayyar Page B.342

Analysis run on: 12/7/99 12:27:42 PM

Input File: C:LFT ProductsLFT ArowiVerificationlverify17.aro

Execution Time $=2.53$ seconds

Total Number Of Pressure Iterations $=0$

Total Number Of Flow Iterations= 2

Total Number Of Enthalpy lterations= 2

Number Of Pipes $=1$

Number Of Junctions $=2$

Length March Solution Method with Mach Number Limits

Segments Per Pipe $=10$

Mach Number Increment= 01

Pressure Tolerance= .0001 relative change

Mass Flow Rate Tolerance= .0001 relative change

Enthalpy Tolerance $=.0001$ relative change

Flow Relaxation $=.5$

Pressure Relaxation $=.5$

Fluid Database: AFT Standard

Fluid= Air

Max Fluid Temperature Data $=1000$ deg. $K$

Min Fluid Temperature Data 200 deg. K

Molecular Weight $=28.97 \mathrm{amu}$

Gas Constant= $0.0685486 \mathrm{Btu} / \mathrm{bm}-\mathrm{R}$

Critical Pressure $=37.25 \mathrm{~atm}$

Critical Temperature= $132.41 \mathrm{deg} . \mathrm{K}$

Acentric Factor $=.021$

Equation of State= Ideal Gas

Enthalpy Model= Roference

Specific Heat Ratio Accuracy= High

Atmospheric Pressure $=1 \mathrm{~atm}$

Gravitational Acceleration= $1 \mathrm{~g}$ 's

Standard Pressure $=14.696 \mathrm{psia}$

Standard Temperature $=60$ deg. F

Turbulent Flow Above Reynolds Number= 4000

Laminar Flow Below Reynolds Number= 2300

Specific Heat Ratio Accuracy= High

Atmospheric Pressure $=1 \mathrm{~atm}$

Gravitational Acceleration $=1 \mathrm{~g}$

Standard Pressure $=14.696$ psia

Standard Temperature $=60$ deg. F

Turbulent Flow Above Reynolds Number $=4000$

Laminar Flow Below Reynolds Number $=2300$

Overall Delta Pressure $=\mathbf{- 2 6 1 . 1 6 1}$ psia

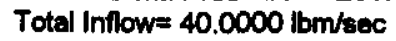

Total Outflow= $40.0000 \mathrm{lbm} / \mathrm{sec}$

Total Energy Inflow= $8299.69 \mathrm{Btu} / \mathrm{s}$

Total Energy Outflow= $8299.69 \mathrm{Btu} / \mathrm{s}$

Total Heat Transferred into System $=0.00000 \mathrm{Btw} / \mathrm{s}$

Maximum Pressure is 257.321 paia at Junction 1 Inlet

Minimum Pressure is 14.7000 psia at Junction 2 Outlet

Maximum Static Temperature is 120.000 deg. F at Junction 2 Inlet

Minimum Static Temperature is $119.986 \mathrm{deg} . \mathrm{F}$ at Junction 1 Inlet

Sonic Choking Exists at Junction 2 (Tank)

Pipe Output Table 
SNF-5331, Rev. 0

(2)

Verify17.aro - Air flow in a pipe, Examplo B8.3 from Nayyar Page B.342

\begin{tabular}{|c|c|c|c|c|c|c|c|c|c|c|c|}
\hline Pipe & $\begin{array}{c}\text { Mass } \\
\text { Flow } \\
\text { (lbm/hr) }\end{array}$ & $\begin{array}{c}\text { Vel. In } \\
\text { (feet/sec) }\end{array}$ & $\begin{array}{l}\text { Vel. Out } \\
\text { (feet/sec) }\end{array}$ & $\begin{array}{c}\text { Mach } \\
\text { \# In }\end{array}$ & $\begin{array}{l}\text { Mach } \\
\text { \# Out }\end{array}$ & $\begin{array}{c}\text { P Static } \\
\text { In } \\
\text { (psia) }\end{array}$ & $\begin{array}{c}\text { P Static } \\
\text { Out } \\
\text { (psia) }\end{array}$ & $\begin{array}{c}\text { T Static } \\
\text { In } \\
\text { (deg. F) }\end{array}$ & $\begin{array}{c}\text { T Static } \\
\text { Out } \\
\text { (deg. F) }\end{array}$ & $\begin{array}{c}\text { T Stag. } \\
\text { In } \\
\text { (deg. F) }\end{array}$ & $\begin{array}{c}\text { TStag. } \\
\text { Out } \\
\text { (deg. F) }\end{array}$ \\
\hline 1 & 144,000 & 370.242 & 1066.13 & 0.317774 & 0.988658 & 257.323 & 76.4630 & 108.750 & 26.6951 & 119.986 & 120.137 \\
\hline
\end{tabular}

\section{All Junction Table}

\begin{tabular}{|c|c|c|c|c|}
\hline Jet & $\begin{array}{c}\text { P Static } \\
\text { In } \\
\text { (psia) }\end{array}$ & $\begin{array}{c}\text { P Static } \\
\text { Out } \\
\text { (psia) }\end{array}$ & $\begin{array}{c}\text { T Static } \\
\text { In } \\
\text { (deg. F) }\end{array}$ & $\begin{array}{c}\text { T Static } \\
\text { Out } \\
\text { (deq. F) }\end{array}$ \\
\hline 1 & 257.321 & 257.3214 & 108.750 & 108.750 \\
\hline 2 & 142.534 & 14.7000 & 120.000 & 120.000 \\
\hline
\end{tabular}


TITLE: VERIFY18.ARO

REFERENCE: Mohinder L. Nayyar, Piping Handbook, Sixth Edition, McGraw-Hill, New York, 1992 , Page B.342, Example B8.4

GAS: Air

ASSUMPTIONS: 1) Adiabatic flow, 2) Perfect gas

RESULTS:

\begin{tabular}{|c|r|r|}
\hline Parameter & Nayyar & AFT Arrow 2.0 \\
\hline$M_{1}-$ Mach number at valve & 0.243 & 0.243 \\
\hline$P_{1}-$ Pressure at valve (psia) & 168.52 & 168.69 \\
\hline
\end{tabular}

\section{DISCUSSION:}

The problem assumes an unusual inlet boundary condition where the flow rate is known and the stagnation temperature. AFT Arrow uses the static temperature at the inlet because it is typically associated with a flow rate. To match the $120 \mathrm{~F}$ stagnation temperature, the inlet static temperature was iterated a few times.

The conditions result in sonic choking at the discharge.

The predictions agree very closely.

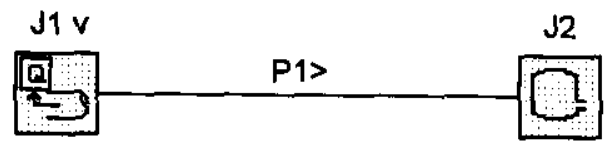

Copyright $\odot 1999$ Applied Flow Technology Corp. All rights reserved.

* Printed with permission. 
Mohinder L. Nayyar

Piping Handbook, Sixth Edftion, MoGraw-Hill, New York, 1992

Page B.342, Example B8.4

Air flow - Example assumes air is a perfect gas and adiabatic flow

See Verify18.doc MS Word file for comparison with published results

Title: Verify18.aro - Air flow in a pipe, Example B8.4 from Nayyar Page B.342

Number Of Pipes $=1$

Number Of Junctions= 2

Length March Solution Method with Mach Number Limits

Segments Per Pipe $=10$

Mach Number Increment= .01

Pressure Tolerance= .0001 relative change

Mass Flow Rate Tolerance $=.0001$ relative change

Enthalpy Tolerancex .0001 relative change

Concentration Tolerance $=.0001$ relative change

Flow Relaxation $=.5$

Pressure Relaxation $=.5$

Fluid Database: AFT Standard

Fluid= Air

Max Fluid Temperature Datas 1000 deg. K

Min Fluid Temperature Data $=200 \mathrm{deg} . \mathrm{K}$

Molecular Weight= $28.97 \mathrm{amu}$

Gas Constant $=0.0685486$ Btu/bm-R

Critical Pressure $=37.25 \mathrm{~atm}$

Critical Temperature $=132.41 \mathrm{deg} . \mathrm{K}$

Acentric Factor $=.021$

Equation of State= Ideal Gas

Enthalpy Model= Reference

Specific Heat Ratio Accuracy= High

Atmospheric Pressure= $1 \mathrm{~atm}$

Gravitational Acceleration 1 g's

Standard Pressure= 14.696 psia

Standard Temperature $=60 \mathrm{deg}$. $F$

Turbulent Flow Above Reynolds Number $=4000$

Laminar Flow Below Reynolds Number= 2300

Specific Heat Ratio Accuracy= High

Atmospheric Pressure $=1 \mathrm{~atm}$

Gravitational Acceleration $=1 \mathrm{~g}$

Standard Pressure $=14.696$ psia

Standard Temperature $=60 \mathrm{deg}$. F

Turbulent Flow Above Reynolds Number= 4000

Laminar Flow Below Reynolds Number= 2300

Pipe Input Table

\begin{tabular}{|c|r|r|r|r|r|r|r|r|r|r|}
\hline Pipe & Name & $\begin{array}{c}\text { Pipe } \\
\text { Defined }\end{array}$ & Length & $\begin{array}{r}\text { Length } \\
\text { Units }\end{array}$ & $\begin{array}{r}\text { Hydraulic } \\
\text { Diameter }\end{array}$ & $\begin{array}{r}\text { Hydraullic } \\
\text { Diam. Units }\end{array}$ & Roughness & $\begin{array}{r}\text { Roughness } \\
\text { Units }\end{array}$ & Losses (K) & Initial Flow \\
\hline 1 & Pipe & Yes & 180 & feet & 4.026 & inches & 0.017 & Explicit f & & 0 \\
\hline
\end{tabular}

\begin{tabular}{|c|r|r|c|r|c|c|c|}
\hline Pipe & $\begin{array}{c}\text { Initial Flow } \\
\text { Units }\end{array}$ & $\begin{array}{r}\text { Junctions } \\
\text { (Up,Down) }\end{array}$ & Geometry & Material & Size & Type & $\begin{array}{r}\text { Special } \\
\text { Condition }\end{array}$ \\
\hline 1 & & 1,2 & Cylindrical Pipe & Steel & 4 inch & schedule 40 & None \\
\hline
\end{tabular}

\section{Assigned Flow Table}


SNF-5331, Rev. 0

AFT Arrow 2.0 Input

Numatec Hanford Corporation
(2)

$12 / 7 / 99$ 12:30 PM

Verify18.aro - Air flow in a pipe, Example B8.4 from Nayyar Page B.342

\begin{tabular}{|c|c|c|c|c|c|c|c|c|c|c|}
\hline Assigned Flow & Name & $\begin{array}{c}\text { Object } \\
\text { Defined }\end{array}$ & $\begin{array}{c}\text { Databese } \\
\text { Source }\end{array}$ & $\begin{array}{c}\text { Special } \\
\text { Condition }\end{array}$ & Fluid & Type & Flow & $\begin{array}{l}\text { Flow } \\
\text { Units }\end{array}$ & $\begin{array}{c}\text { Temperature } \\
\text { Temperature } \\
\text { Units }\end{array}$ \\
\hline 1 & Assigned Flow & Yes & & None & Air & Inflow & 72000 & $\mathrm{lbm} / \mathrm{hr}$ & 113.5 & $\mathrm{deg} . \mathrm{F}$ \\
\hline
\end{tabular}

\section{Tank Table}

\begin{tabular}{|c|r|r|r|r|r|r|r|r|r|r|}
\hline Tank & Name & $\begin{array}{c}\text { Object } \\
\text { Defined }\end{array}$ & $\begin{array}{c}\text { Database } \\
\text { Source }\end{array}$ & $\begin{array}{c}\text { Special } \\
\text { Condition }\end{array}$ & Fluid & Pressure & $\begin{array}{c}\text { Pressure } \\
\text { Units }\end{array}$ & Temperature & $\begin{array}{c}\text { Temperature } \\
\text { Units }\end{array}$ & $\begin{array}{c}\text { Balance } \\
\text { Energy }\end{array}$ \\
\hline 2 & Tank & Yes & & N/A & Air & 14.7 & psia & 120 & deg. F & No \\
\hline
\end{tabular}

\begin{tabular}{|c|c|c|c|c|c|c|c|c|c|}
\hline Tank & $\begin{array}{c}\text { Balance } \\
\text { Concentration }\end{array}$ & $\begin{array}{c}\text { (Pipe \#1) } \\
\mathrm{K} \ln , \mathrm{K} \text { Out }\end{array}$ & $\begin{array}{c}\text { (Pipe *2) } \\
\mathrm{K} \ln , \mathrm{K} \text { Out }\end{array}$ & $\begin{array}{c}\text { (Pipe *3) } \\
\mathrm{K} \ln , \mathrm{K} \text { Out }\end{array}$ & $\begin{array}{c}\text { (Pipe *4) } \\
\mathrm{K} \ln , \mathrm{K} \text { Out }\end{array}$ & $\begin{array}{c}\text { (Pipe \#5) } \\
\mathrm{K} \ln , \mathrm{K} \text { Out }\end{array}$ & $\begin{array}{c}\text { (Pipe \#6) } \\
\mathrm{K} \ln , \mathrm{K} \text { Out }\end{array}$ & $\begin{array}{c}\text { (Pipe \#7) } \\
\mathrm{K} \ln , \mathrm{K} \text { Out }\end{array}$ & $\begin{array}{c}\text { (Pipe \#8) } \\
\mathrm{K} \ln , \mathrm{K} \text { Out }\end{array}$ \\
\hline 2 & No & $(1) 0,0$ & & & & & & \\
\hline
\end{tabular}

\begin{tabular}{|c|c|c|}
\hline Tank & $\begin{array}{c}\text { (Pipe \#9) } \\
\mathrm{K} \ln , \mathrm{K} \text { Out }\end{array}$ & $\begin{array}{c}\text { (Pipe } \text { K10) } \\
\mathrm{K} \text { In, K Out }\end{array}$ \\
\hline 2 & & \\
\hline
\end{tabular}


Mohinder L. Nayyar

Piping Handbook, Sixth Edition, McGraw-Hill, New York, 1992

Page B.342, Example B8.4

Air flow - Example assumes air is a perfect gas and adiabatic flow

See Verify18.doc MS Word file for comparison with published results

Title: Verify18.aro - Air flow in a pipe, Example B8.4 from Nayyar Page B.342

Analysis run on: 127/99 12:29:24 PM

Input File: C:VFT ProductsLFT ArrowlVerificationiverify18.aro

Execution Time= 3.13 seconds

Total Number of Pressure Herations $=0$

Total Number Of Flow lterations $=2$

Total Number Of Enthalpy Iterations= 2

Number Of Pipes= 1

Number of Junctions= 2

Length March Solution Method with Mach Number Limits

Segments Per Pipe= 10

Mach Number increment= .01

Pressure Tolerance= .0001 relative change

Mass Flow Rate Tolerance= .0001 relative change

Enthalpy Tolerance=, 0001 relative change

Flow Relaxation= .5

Pressure Relaxationx .5

Fluid Database: AFT Standard

Fluid= Air

Max Fluid Temperature Data $=1000$ deg. K

Min Fluid Temperature Data $=200$ deg. K

Molecular Weight= $28.97 \mathrm{amu}$

Gas Constant $=0.0685486 \mathrm{Btu} / \mathrm{bm}-\mathrm{R}$

Critical Pressure= 37.25 atm

Critical Temperature= $132.41 \mathrm{deg} . \mathrm{K}$

Acentric Factor $=.021$

Equation of State= Ideal Gas

Enthalpy Model= Reference

Specific Hoat Ratio Accuracy= High

Atmospheric Pressure= $1 \mathrm{~atm}$

Gravitational Acceleration= $1 \mathrm{~g}$ 's

Standard Pressure $=14.696$ psia

Standard Temperature $=60 \mathrm{deg} . F$

Turbulent Flow Above Reynolds Number= 4000

Laminar Flow Below Reynolds Number= 2300

Specific Heat Ratio Accuracy= High

Atmospheric Pressure= $1 \mathrm{~atm}$

Gravitational Acceleration $1 \mathrm{~g}$

Standard Pressure $=14.696$ psia

Standard Temperature $=60$ deg. F

Turbulent Flow Above Reynolds Number= $\mathbf{4 0 0 0}$

Laminar Flow Below Reynolds Number= 2300

Overall Delta Pressure $=-161.046$ psia

Total Inflow $=20.0000 \mathrm{lbm} / \mathrm{sec}$

Total Outflow $=20.0000 \mathrm{lbm} / \mathrm{sec}$

Total Energy Inflow= 4150.63 Btu/s

Total Energy Outflow= 4150.63 Btu/s

Total Heat Transferred Into System $=0.00000 \mathrm{Btu} / \mathrm{s}$

Maximum Pressure is 168.690 psia at Junction 1 Inlet

Minimum Pressure is 14.7000 psia at Junction 2 Outlet

Maximum Static Temperature is 120.145 deg. F at Junction 1 Inlet

Minimum Static Temperature is 120.000 deg. $F$ at Junetion 2 Inlet

Sonic Choking Exists at Junction 2 (Tank)

\section{Pipe Output Table}


SNF-5331, Rev. 0

AFT Arrow 2.0 Output

Numatec Hanford Corporation

(2)

$12 / 7 / 99$

Verify18.aro - Air flow in a pipe, Exemple B8.4 from Nayyar Page B.342

\begin{tabular}{|c|c|c|c|c|c|c|c|c|c|c|c|}
\hline Pipe & $\begin{array}{l}\text { Mass } \\
\text { Flow } \\
(\text { lbm/hr) }\end{array}$ & $\begin{array}{c}\text { Vel. In } \\
\text { (feet/sec) }\end{array}$ & $\begin{array}{l}\text { Vel. Out } \\
\text { (feet/oec) }\end{array}$ & $\begin{array}{c}\text { Mach } \\
\text { \# In }\end{array}$ & $\begin{array}{l}\text { Mach } \\
\text { \# Out }\end{array}$ & $\begin{array}{c}\text { P Static } \\
\text { In } \\
\text { (psia) }\end{array}$ & $\begin{array}{c}\text { P Static } \\
\text { Out } \\
\text { (psia) }\end{array}$ & $\begin{array}{c}\text { T Static } \\
\text { In } \\
\text { (deg. F) }\end{array}$ & $\begin{array}{c}\text { T Static } \\
\text { Out } \\
\text { (deg. F) }\end{array}$ & $\begin{array}{c}\text { T Stag. } \\
\text { In } \\
\text { (deg. F) }\end{array}$ & $\begin{array}{c}\text { T Stag. } \\
\text { Out } \\
\text { (deg. F) }\end{array}$ \\
\hline 1 & $72,000.0$ & 284.747 & 1070.27 & 0.243388 & 0.993082 & 168.690 & 38.0391 & 113.500 & 26.1325 & 120.145 & 120.300 \\
\hline
\end{tabular}

\section{All Junction Table}

\begin{tabular}{|c|c|c|c|c|}
\hline Jet & $\begin{array}{c}\text { P Static } \\
\text { In } \\
\text { (psia) }\end{array}$ & $\begin{array}{c}\text { P Static } \\
\text { Out } \\
\text { (psia) }\end{array}$ & $\begin{array}{c}\text { T Static } \\
\text { In } \\
\text { (deq. F) }\end{array}$ & $\begin{array}{c}\text { T Static } \\
\text { Out } \\
\text { (der. F) }\end{array}$ \\
\hline 1 & 168.6898 & 168.6898 & 113.500 & 113.500 \\
\hline 2 & 71.2718 & 14.7000 & 120.000 & 120.000 \\
\hline
\end{tabular}


TITLE: VERIFY19.ARO

REFERENCE: Mohinder L. Nayyar, Piping Handbook, Sixth Edition, McGraw-Hill, New York, 1992, Page B.342, Example B8.5

GAS: Air

ASSUMPTIONS: 1) Adiabatic flow, 2) Perfect gas

RESULTS:

\begin{tabular}{|l|r|r|}
\hline & Nayy ar & AFT Arrow 2.0 \\
\hline$P_{1}-$ Pressure at valve (psia) & 165.29 & 165.60 \\
\hline
\end{tabular}

\section{DISCUSSION:}

The problem assumes an unusual inlet boundary condition where the flow rate is known and the stagnation temperature. AFT Arrow uses the static temperature at the inlet because it is typically associated with a flow rate. To match the $500 \mathrm{~F}$ stagnation temperature, the inlet static temperature was iterated a few times.

The conditions result in sonic choking at the discharge.

The predictions agree very closely.

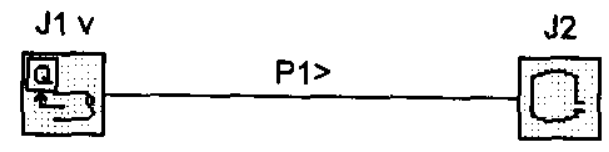

Copyright $\bigcirc 1999$ Applied Flow Technology Corp. All rights reserved. 
SNF-5331, Rev. 0

AFT Arrow 2.0 input

Numatec Hanford Corporation

(1)

$12 / 20 / 99$ 10:41 AM

Verify19.aro - Air flow in a pipe, Example B8.5 from Nayyar Page B.342

Mohinder L. Nayyar

Piping Handbook, Sixth Edition, McGraw-Hill, New York, 1992

Page B.342, B.343, Example B8.5

Air flow - Example assumes air is a perfect gas and adiabatic flow

See Verify 19.doc MS Word file for comparison with published results

Title: Verify19.aro - Air flow in a pipe, Example B8.5 from Nayyar Page B.342

Number Of Pipes $=1$

Number Of Junctions $=2$

Length March Solution Method with Mach Number Limits

Segments Per Pipe= 10

Mach Number Increment= 01

Pressure Tolerance $=.0001$ relative change

Mass Flow Rate Tolerance $=.0001$ relative change

Enthalpy Tolerance= .0001 relative change

Flow Relaxation $=.5$

Pressure Relaxation $=.5$

Fluid Database: AFT Standard

Fluid= Air

Max Fluid Temperature Data $=1000$ deg. $K$

Min Fluid Temperature Data $=200 \mathrm{deg} . \mathrm{K}$

Molecular Weight $=28.97 \mathrm{amu}$

Gas Constant= 0.0685486 Btu/fbm-R

Critical Pressure= 37.25 atm

Critical Temperature= 132.41 deg. $\mathrm{K}$

Acentric Factor $=.021$

Equation of State= Ideal Gas

Enthalpy Model= Reference

Specific Heat Ratio Accuracy= High

Atmospheric Pressure= $1 \mathrm{~atm}$

Gravitational Acceleration $=1 \mathrm{~g}$ 's

Standard Pressure $=14.696$ psia

Standard Temperature $=60$ deg. $F$

Turbulent Flow Above Reynolds Number $=4000$

Laminar Flow Below Reynolds Number $=2300$

Specific Heat Ratio Accuracy= High

Atmospheric Pressure= 1 atm

Gravitational Acceleration $=1 \mathrm{~g}$

Standard Pressure= 14.696 psia

Standard Temperature $=60$ deg. $F$

Turbulent Flow Above Reynolds Number $=4000$

Laminar Flow Below Reynolds Number $=\mathbf{2 3 0 0}$

Pipe Input Table

\begin{tabular}{|c|r|r|r|r|r|r|r|r|r|r|}
\hline Pipe & Name & $\begin{array}{c}\text { Pipe } \\
\text { Defined }\end{array}$ & Length & $\begin{array}{r}\text { Length } \\
\text { Units }\end{array}$ & $\begin{array}{r}\text { Hydraulic } \\
\text { Diameter }\end{array}$ & $\begin{array}{c}\text { Hydraulic } \\
\text { Diam. Units }\end{array}$ & Roughness & $\begin{array}{r}\text { Roughness } \\
\text { Units }\end{array}$ & Losses (K) & Initial Flow \\
\hline 1 & Pipe & Yes & 90 & feet & 4.026 & inches & 0.017 & Explicit f & 0 & \\
\hline
\end{tabular}

\begin{tabular}{|c|c|r|c|c|c|c|c|}
\hline Pipe & $\begin{array}{c}\text { Initial Flow } \\
\text { Units }\end{array}$ & $\begin{array}{r}\text { Junctions } \\
\text { (Up,Down) }\end{array}$ & Geometry & Material & Size & Type & $\begin{array}{c}\text { Special } \\
\text { Condition }\end{array}$ \\
\hline 1 & & 1,2 & Cylindrical Pipe & Steel & 4 inch & schedule 40 & None \\
\hline
\end{tabular}

\section{Assigned Flow Table}


SNF-5331, Rev. 0

AFT Arrow 2.0 lnput

(2)

12/20/99 10:41 AM

Numatec Hanford Corporation

Verify 19.aro - Air flow in a pipe, Example B8.5 from Nayyar Page B.342

\begin{tabular}{|c|c|c|c|c|c|c|c|c|c|c|}
\hline Assigned Flow & Name & $\begin{array}{c}\text { Object } \\
\text { Defined }\end{array}$ & $\begin{array}{c}\text { Database } \\
\text { Source }\end{array}$ & $\begin{array}{c}\text { Special } \\
\text { Condition }\end{array}$ & Fluid & Type & Flow & $\begin{array}{r}\text { Flow } \\
\text { Units }\end{array}$ & $\begin{array}{c}\text { Temperature } \\
\text { Temperature } \\
\text { Units }\end{array}$ \\
\hline 1 & Assigned Flow & Yes & & None & Air & Inflow & 72000 & lbm/hr & 481 & deg. F \\
\hline
\end{tabular}

\begin{tabular}{|c|r|}
\hline Assigned Flow & $\begin{array}{c}\text { Loss } \\
\text { Model }\end{array}$ \\
\hline 1 & 0 \\
\hline
\end{tabular}

Tank Table

\begin{tabular}{|c|r|r|r|r|r|r|r|r|r|r|}
\hline Tank & Name & $\begin{array}{c}\text { Object } \\
\text { Defined }\end{array}$ & $\begin{array}{c}\text { Database } \\
\text { Source }\end{array}$ & $\begin{array}{c}\text { Special } \\
\text { Condition }\end{array}$ & Fluid & Pressure & $\begin{array}{r}\text { Pressure } \\
\text { Units }\end{array}$ & Temperature & $\begin{array}{r}\text { Temperature } \\
\text { Units }\end{array}$ & $\begin{array}{c}\text { Balance } \\
\text { Energy }\end{array}$ \\
\hline 2 & Tank & Yes & & N/A & Air & 14.7 & psia & 120 & deg. F & No \\
\hline
\end{tabular}

\begin{tabular}{|c|c|c|c|c|c|c|c|c|c|}
\hline Tank & $\begin{array}{c}\text { Balance } \\
\text { Concentration }\end{array}$ & $\begin{array}{c}\text { (Pipe \#1) } \\
\mathrm{K} \ln , \mathrm{K} \text { Out }\end{array}$ & $\begin{array}{c}\text { (Pipe *2) } \\
\mathrm{K} \ln , \mathrm{K} \text { Out }\end{array}$ & $\begin{array}{c}\text { (Pipe \#3) } \\
\mathrm{K} \ln , \mathrm{K} \text { Out }\end{array}$ & $\begin{array}{c}\text { (Pipe \#4) } \\
\mathrm{K} \ln , \mathrm{K} \text { Out }\end{array}$ & $\begin{array}{c}\text { (Pipe \#5) } \\
\mathrm{K} \ln , \mathrm{K} \text { Out }\end{array}$ & $\begin{array}{c}\text { (Pipe \#6) } \\
\mathrm{K} \ln , \mathrm{K} \text { Out }\end{array}$ & $\begin{array}{c}\text { (Pipe \#7) } \\
\mathrm{K} \ln , \mathrm{K} \text { Out }\end{array}$ & $\begin{array}{c}\text { (Pipe \#8) } \\
\mathrm{K} \ln , \mathrm{K} \text { Out }\end{array}$ \\
\hline $\mathbf{2}$ & $\mathrm{No}$ & $(1) 0,0$ & & & & & & \\
\hline
\end{tabular}

\begin{tabular}{|c|c|c|}
\hline Tank & $\begin{array}{c}\text { (Pipe *9) } \\
\text { KIn, K Out }\end{array}$ & $\begin{array}{c}\text { (Pipe } \# 10) \\
\text { KIn, K Out }\end{array}$ \\
\hline 2 & & \\
\hline
\end{tabular}


Mohinder L. Nayyar

Piping Handbook, Sixth Edition, McGraw-Hill, New York, 1992

Page B.342, B.343, Example B8.5

Air flow - Example assumes air is a perfect gas and adiabatic flow

See Verify19, doc MS Word file for comparison with published results

Title: Verify19.aro - Air flow in a pipe, Example B8.5 from Nayyar Page B.342

Analysis run on: 12/20/99 10:40:24 AM

Input File: C:AFT ProductsLAFT ArrowlVerificationiverity19.aro

Execution Time $=3.35$ seconds

Total Number Of Pressure lterations= 0

Total Number Of Flow lterations $=2$

Total Number Of Enthalpy Iterations= 2

Number Of Pipes= 1

Number of Junctions= 2

Length March Solution Method with Mach Number Limits

Segments Per Pipe $=10$

Mach Number Increment= .01

Pressure Tolerance $=.0001$ relative change

Mass Flow Rate Tolerance= .0001 relative change

Enthalpy Tolerance $=0001$ relative change

Flow Relaxation $=.5$

Pressure Relaxation $=.5$

Fluid Database: AFT Standard

Fluid= Air

Max Fluid Temperature Data $=1000$ deg. K

Min Fluid Temperature Data $=\mathbf{2 0 0}$ deg. K

Molecular Weight= $28.97 \mathrm{amu}$

Gas Constant= 0.0685486 Btu/lbm-R

Critical Pressure $=37.25 \mathrm{~atm}$

Critical Temperature $=132.41$ deg. $K$

Acentric Factorm, 021

Equation of State= Ideal Gas

Enthalpy Model= Reference

Specific Heat Ratio Accuracy= High

Atmospheric Pressure= $1 \mathrm{~atm}$

Gravitational Acceleration $=1 \mathrm{~g}$ 's

Standard Pressure $=14.696$ psia

Standard Temperature $=60 \mathrm{deg} . F$

Turbulent Flow Above Reynolds Number $=4000$

Laminar Flow Below Reynolds Number= 2300

Specific Heat Ratio Accuracy= High

Atmospheric Pressure $=1 \mathrm{~atm}$

Gravitational Acceleration $=1 \mathrm{~g}$

Standard Pressure $=14.696$ psia

Standard Temperature $=60$ deg. F

Turbulent Flow Above Reynolds Number $=\mathbf{4 0 0 0}$

Laminar Flow Below Reynolds Number $\mathbf{2 3 0 0}$

Overall Delta Pressure $=-162.824$ psia

Total inflow $=20.0000 \mathrm{lbm} / \mathrm{sec}$

Total Outflow $20.0000 \mathrm{lbm} / \mathrm{sec}$

Total Energy Inflow= 6011.31 Btu/s

Total Energy Outflow= $6011.31 \mathrm{Btw} / \mathrm{s}$

Total Heat Transferred Into System $=0.00000 \mathrm{Btu} / \mathrm{s}$

Maximum Pressure is 165.604 psia at Junction 1 Inlet

Minimum Pressure is 14.7000 psia at Junction 2 Outlet

Maximum Static Temperature is $499.335 \mathrm{deg}$. F at Junction 1 Inlet

Minimum Static Temperature is $120.000 \mathrm{deg}$. F at Junction 2 Inlet

Sonic Choking Exists at Junction 2 (Tank)

\section{Pipe Output Table}


Verify19.aro - Air flow in a pipe, Example B8.5 from Nayyar Page B.342

\begin{tabular}{|c|c|c|c|c|c|c|c|c|c|c|c|}
\hline Pipe & $\begin{array}{c}\text { Mass } \\
\text { Flow } \\
\text { (lbm/hr) }\end{array}$ & $\begin{array}{c}\text { Vel. In } \\
\text { (feetsec) }\end{array}$ & $\begin{array}{l}\text { Vel. Out } \\
\text { (feet/sec) }\end{array}$ & $\begin{array}{c}\text { Mach } \\
* \text { In }\end{array}$ & $\begin{array}{l}\text { Mach } \\
\text { \# Out }\end{array}$ & $\begin{array}{c}\text { P Static } \\
\text { In } \\
\text { (psia) }\end{array}$ & $\begin{array}{c}\text { P Static } \\
\text { Out } \\
\text { (psia) }\end{array}$ & $\begin{array}{c}\text { T Static } \\
\text { In } \\
\text { (deg. F) }\end{array}$ & $\begin{array}{c}\text { T Static } \\
\text { Out } \\
\text { (deg. F) }\end{array}$ & $\begin{array}{c}\text { T Stag. } \\
\text { In } \\
\text { (deg. F) }\end{array}$ & $\begin{array}{c}\text { T Stag. } \\
\text { Out } \\
\text { (deg. F) }\end{array}$ \\
\hline 1 & $72,000.0$ & 476.028 & 1377.07 & 0.318401 & 0.994614 & 165.604 & 49.0050 & 481.000 & 345.576 & 499.335 & 499.740 \\
\hline
\end{tabular}

All Junction Table

\begin{tabular}{|c|c|c|c|c|}
\hline Jct & $\begin{array}{c}\text { P Static } \\
\text { In } \\
\text { (psia) }\end{array}$ & $\begin{array}{c}\text { P Static } \\
\text { Out } \\
\text { (psia) }\end{array}$ & $\begin{array}{c}\text { T Static } \\
\text { In } \\
\text { (deg. F) }\end{array}$ & $\begin{array}{c}\text { T Static } \\
\text { Out } \\
\text { (deg. F) }\end{array}$ \\
\hline 1 & 165.6037 & 165.6037 & 481.000 & 481.000 \\
\hline 2 & 91.8048 & 14.7000 & 120.000 & 120.000 \\
\hline
\end{tabular}


TITLE: VERIFY20.ARO

REFERENCE: Mohinder L. Nayyar, Piping Handbook, Sixth Edition, McGraw-Hill, New York, 1992, Page B.343, Example B8.6

GAS: Air

ASSUMPTIONS: 1) Adiabatic flow, 2) Perfect gas

RESULTS:

\begin{tabular}{|l|r|r|}
\hline \multicolumn{1}{|c|}{ Parameter } & Nayyar & AFT Arrow 2.0 \\
\hline$M_{1}$-Mach number at valve & 0.235 & 0.235 \\
\hline$P_{1}-$ Pressure at valve (psia) & 661.96 & 662.56 \\
\hline
\end{tabular}

\section{DISCUSSION:}

The problem assumes an unusual inlet boundary condition where the flow rate is known and the stagnation temperature. AFT Arrow uses the static temperature at the inlet because it is typically associated with a flow rate. To match the $120 \mathrm{~F}$ stagnation temperature, the inlet static temperature was iterated a few times.

The conditions result in sonic choking at the discharge.

The predictions agree very closely.

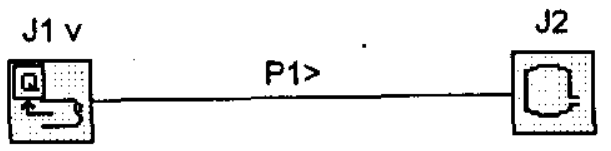

Copyright $\bigcirc 1999$ Applied Flow Technology Corp. All rights reserved. 
AFT Arrow 2.0 Input

Numatec Hanford Corporation
(1)

$127 / 99$ 12:39 PM

Verify20.aro - Air flow in a pipe, Example B8.6 from Nayyar Page B.343

Mohinder L. Nayyar

Piping Handbook, Sixth Edition, McGraw-Hill, New York, 1992

Page B.343, Example B8.6

Air flow - Example assumes air is a perfect gas and adiabatic flow

See Verify20.doc MS Word file for comparison with published results

Title: Verify20.aro - Air flow in a pipe, Example B8.6 from Nayyar Page B.343

Number Of Pipes $=1$

Number Of Junctions $=2$

Length March Solution Method with Mach Number Limits

Segments Per Pipe $=10$

Mach Number Increment= .01

Pressure Tolerance $=.0001$ relative change

Mass Flow Rate Tolerance $=.0001$ relative change

Enthalpy Tolerance $=.0001$ relative change

Flow Relaxation $=.5$

Pressure Relaxation $=.5$

Fluid Database: AFT Standard

Fluid= Air

Max Fluid Temperature Data $=1000$ deg. $\mathrm{K}$

Min Fluid Temperature Data $=200$ deg. K

Molecular Weight $=28.97 \mathrm{amu}$

Gas Constant $=0.0685486 \mathrm{Btu} / \mathrm{bm}-\mathrm{R}$

Critical Pressure $=37.25 \mathrm{~atm}$

Critical Temperature $=132.41 \mathrm{deg} . \mathrm{K}$

Acentric Factor $=.021$

Equation of State $=$ Ideal Gas

Enthalpy Model= Reference

Specific Heat Ratio Accuracy $=$ High

Atmospheric Pressure $=1 \mathrm{~atm}$

Gravitational Acceleration $=1 \mathrm{~g}$ 's

Standard Pressure $=14.696$ psia

Standard Temperature $=60$ deg. $F$

Turbulent Flow Above Reynolds Number $=4000$

Laminar Flow Below Reynolds Number $=2300$

Specific Heat Ratio Accuracy= High

Atmospheric Pressure $=1 \mathrm{~atm}$

Gravitational Acceleration $=1 \mathrm{~g}$

Standard Pressure $=14.696$ psia

Standard Temperature $=60$ deg. $F$

Turbulent Flow Above Reynolds Number $=4000$

Laminar Flow Below Reynolds Number $=\mathbf{2 3 0 0}$

Pipe Input Table

\begin{tabular}{|c|r|r|r|r|r|r|r|r|r|r|}
\hline Pipe & Name & $\begin{array}{c}\text { Pipe } \\
\text { Defined }\end{array}$ & Length & $\begin{array}{r}\text { Length } \\
\text { Units }\end{array}$ & $\begin{array}{r}\text { Hydraulic } \\
\text { Diameter }\end{array}$ & $\begin{array}{c}\text { Hydraulic } \\
\text { Diam. Units }\end{array}$ & Roughness & $\begin{array}{c}\text { Roughness } \\
\text { Units }\end{array}$ & Losses (K) & Initial Flow \\
\hline 1 & Pipe & Yes & 90 & feet & 2.067 & inches & 0.019 & Explicit $f$ & 0 & \\
\hline
\end{tabular}

\begin{tabular}{|c|c|r|c|r|c|c|c|}
\hline Pipe & $\begin{array}{c}\text { Initial Flow } \\
\text { Units }\end{array}$ & $\begin{array}{c}\text { Junctions } \\
(\text { Up,Down })\end{array}$ & Geometry & Material & Size & Type & $\begin{array}{c}\text { Special } \\
\text { Condition }\end{array}$ \\
\hline 1 & & 1,2 & Cylindrical Pipe & Steel & 2 inch & schedule 40 & None \\
\hline
\end{tabular}

\section{Assigned Flow Table}


SNF-5331, Rev. 0

AFT Arrow 2.0 Input

(2)

12//99 12:39 PM

Numatec Hanford Corporation

Verify20.aro - Air flow in a pipe, Example B8.6 from Nayyar Page B.343

\begin{tabular}{|c|c|c|c|c|c|c|c|c|r|r|}
\hline Assigned Flow & Name & $\begin{array}{c}\text { Object } \\
\text { Defined }\end{array}$ & $\begin{array}{c}\text { Database } \\
\text { Source }\end{array}$ & $\begin{array}{c}\text { Special } \\
\text { Condition }\end{array}$ & Fluid & Type & Flow & $\begin{array}{c}\text { Flow } \\
\text { Units }\end{array}$ & $\begin{array}{r}\text { Temperature } \\
\text { Temperature } \\
\text { Units }\end{array}$ \\
\hline 1 & Assigned Flow & Yes & & None & Air & Inflow & 72000 & lbm/hr & 113.75 & deg. F \\
\hline
\end{tabular}

\begin{tabular}{|c|r|}
\hline Assigned Flow & $\begin{array}{c}\text { Loss } \\
\text { Model }\end{array}$ \\
\hline 1 & 0 \\
\hline
\end{tabular}

Tank Table

\begin{tabular}{|c|r|r|r|r|r|r|r|r|r|r|}
\hline Tank & Name & $\begin{array}{c}\text { Object } \\
\text { Defined }\end{array}$ & $\begin{array}{c}\text { Database } \\
\text { Source }\end{array}$ & $\begin{array}{c}\text { Special } \\
\text { Condition }\end{array}$ & Fluid & Pressure & $\begin{array}{c}\text { Pressure } \\
\text { Units }\end{array}$ & $\begin{array}{r}\text { Temperature } \\
\text { Temperature } \\
\text { Units }\end{array}$ & $\begin{array}{c}\text { Balance } \\
\text { Energy }\end{array}$ \\
\hline $\mathbf{2}$ & Tank & Yes & & N/A & Air & 14.7 & psia & 120 & deg. F & No \\
\hline
\end{tabular}

\begin{tabular}{|c|c|c|c|c|c|c|c|c|c|}
\hline Tank & $\begin{array}{c}\text { Balance } \\
\text { Concentration }\end{array}$ & $\begin{array}{c}\text { (Pipe \#1) } \\
\text { KIn, K Out }\end{array}$ & $\begin{array}{c}\text { (Pipe \#2) } \\
\text { KIn, K Out }\end{array}$ & $\begin{array}{c}\text { (Pipe \#3) } \\
\text { KIn, K Out }\end{array}$ & $\begin{array}{c}\text { (Pipe \#4) } \\
\text { K In, K Out }\end{array}$ & $\begin{array}{c}\text { (Pipe \#5) } \\
K \ln , K \text { Out }\end{array}$ & $\begin{array}{c}\text { (Pipe \#6) } \\
K \ln , K \text { Out }\end{array}$ & $\begin{array}{c}\text { (Pipe \#7) } \\
\text { K In, K Out }\end{array}$ & $\begin{array}{c}\text { (Pipe \#8) } \\
\text { KIn, K Out }\end{array}$ \\
\hline 2 & No & (1) 0,0 & & & & & & & \\
\hline
\end{tabular}

\begin{tabular}{|c|c|c|}
\hline Tank & $\begin{array}{c}\text { (Pipe \#9) } \\
\text { KIn, K Out }\end{array}$ & $\begin{array}{c}\text { (Pipe \#10) } \\
\text { KIn, K Out }\end{array}$ \\
\hline 2 & & \\
\hline
\end{tabular}


Mohinder L. Nayyar

Piping Handbook, Sixth Edition, MoGraw-Hill, Now York, 1992

Page B.343, Example B8.6

Air flow - Example assumes air is a perfect gas and adiabatic flow

See Verify20.doc MS Word file for comparison with published results

Title: Verify20.aro - Air flow in a pipe, Example B8.6 from Nayyar Page B.343

Analysis run on: 12/799 12:37:36 PM

Input File: C:LFT ProductsLAFT ArrowVerificationlverify20.aro

Execution Time $=2.74$ seconds

Total Number Of Pressure lterations $=0$

Total Number Of Flow Iterations= 2

Total Number Of Enthalpy Iterations= 2

Number Of Pipes $=1$

Number Of Junctions= 2

Length March Solution Method with Mach Number Limits

Segments Per Pipe $=10$

Mach Number Increment= 01

Pressure Tolerances .0001 relative change

Mass Flow Rate Tolerance .0001 relative change

Enthalpy Tolerance .0001 relative change

Fiow Relaxation= .5

Pressure Relaxation $=.5$

Fluid Database: AFT Standard

Fluid= Air

Max Fluid Temperature Data $=1000$ deg. $K$

Min Fluid Temperature Data $=200$ deg. K

Molecular Weight $=28.97$ amu

Gas Constant $=0.0685486 \mathrm{Btu} / \mathrm{bm}-\mathrm{R}$

Critical Pressure $=37.25 \mathrm{~atm}$

Critical Temperature $=132.41 \mathrm{deg} . \mathrm{K}$

Acentric Factor $=.021$

Equation of State= Ideal Gas

Enthalpy Model= Reference

Specific Heat Ratio Accuracy= High

Atmospheric Pressure $=1 \mathrm{~atm}$

Gravitational Acceleration $=1 \mathrm{~g}$ 's

Standard Pressure= 14.696 psia

Standard Temperature= $60 \mathrm{deg}$. F

Turbulent Flow Above Reynolds Number= $\mathbf{4 0 0 0}$

Laminar Flow Below Reynolds Number= 2300

Specific Heat Ratio Accuracy= High

Atmospheric Pressure= $1 \mathrm{~atm}$

Gravitational Acceleration= $1 \mathrm{~g}$

Standard Pressure= 14.696 psia

Standard Temperature $=60 \mathrm{deg} . \mathrm{F}$

Turbulent Flow Above Reynolds Number= 4000

Laminar Flow Below Reynolds Number= 2300

Overall Delta Pressure $=-673.699$ psia

Total Inflow= $20.0000 \mathrm{lbm} / \mathrm{sec}$

Total Outflow $=20.0000 \mathrm{lbm} / \mathrm{sec}$

Total Energy Inflow= 4149.70 Btu/s

Total Energy Outflow= $4149.70 \mathrm{Btu} / \mathrm{s}$

Total Heat Transferred Into System= $0.00000 \mathrm{Btu} / \mathrm{s}$

Maximum Pressure is 662.559 psia at Junction 1 Inlet Minimum Pressure is 14.7000 psia at Junction 2 Outlet

Maximum Static Temperature is 120.000 deg. F at Junction 2 Inlet

Minimum Static Temperature is 119.955 deg. F at Junction 1 Inlet

Sonic Choking Exists at Junction 2 (Tank) 
SNF-5331, Rev. 0

(2)

Verify20.aro - Air flow in a pipe, Example B8.6 from Nayyar Page B.343

\begin{tabular}{|c|c|c|c|c|c|c|c|c|c|c|c|}
\hline Pip & $\begin{array}{l}\text { Mass } \\
\text { Flow } \\
\text { (lbm/hr) }\end{array}$ & $\begin{array}{c}\text { Vel. In } \\
\text { (feet/sec) }\end{array}$ & $\begin{array}{l}\text { Vel. Out } \\
\text { (feet/sec) }\end{array}$ & $\begin{array}{c}\text { Mach } \\
\# \ln \end{array}$ & $\begin{array}{l}\text { Mach } \\
\# \text { Out }\end{array}$ & $\begin{array}{c}\text { P Static } \\
\text { In } \\
\text { (psia) }\end{array}$ & $\begin{array}{c}\text { P Static } \\
\text { Out } \\
\text { (psia) }\end{array}$ & $\begin{array}{c}\text { T Static } \\
\text { In } \\
\text { (deg. F) }\end{array}$ & $\begin{array}{c}\text { T Static } \\
\text { Out } \\
\text { (deg. F) }\end{array}$ & $\begin{array}{c}\text { T Stag. } \\
\text { In } \\
(\operatorname{deg}, F)\end{array}$ & $\begin{array}{c}\text { T Stag. } \\
\text { Out } \\
\text { (deg. F) }\end{array}$ \\
\hline A & $72,000.0$ & 275.155 & 1062.54 & 0.235138 & 0.984739 & 662.563 & 145.708 & 113.750 & 27.2966 & 119.955 & 120.105 \\
\hline
\end{tabular}

\section{All Junction Table}

\begin{tabular}{|c|c|c|c|c|}
\hline Jet & $\begin{array}{c}\text { P Static } \\
\text { in } \\
\text { (psia) }\end{array}$ & $\begin{array}{c}\text { P Static } \\
\text { Out } \\
\text { (psia) }\end{array}$ & $\begin{array}{c}\text { T Static } \\
\text { In } \\
\text { (deg. F) }\end{array}$ & $\begin{array}{c}\text { T Static } \\
\text { Out } \\
\text { (deg. F) }\end{array}$ \\
\hline 1 & 662.559 & 662.5590 & 113.750 & 113.750 \\
\hline 2 & 270.384 & 14.7000 & 120.000 & 120.000 \\
\hline
\end{tabular}


TITLE: VERIFY21.ARO

REFERENCE: Mohinder L. Nayyar, Piping Handbook, Sixth Edition, McGraw-Hill, New York, 1992, Page B.343-344, Example B8.7

GAS: Air

ASSUMPTIONS: 1) Adiabatic flow, 2) Perfect gas

RESULTS:

\begin{tabular}{|l|r|r|}
\hline \multicolumn{1}{|c|}{ Parameter } & \multicolumn{1}{|c|}{ Nayyar } & AFT Arrow 2.0 \\
\hline$M_{1}-$ Mach number at valve & 0.4096 & 0.4142 \\
\hline$P_{1}-$ Pressure at valve (psia) & 25.15 & 24.95 \\
\hline
\end{tabular}

\section{DISCUSSION:}

The problem assumes an unusual inlet boundary condition where the flow rate is known and the stagnation temperature. AFT Arrow uses the static temperature at the inlet because it is typically associated with a flow rate. To match the $120 \mathrm{~F}$ stagnation temperature, the inlet static temperature was iterated a few times.

The result is sub-sonic conditions at the discharge.

The predictions agree very closely.

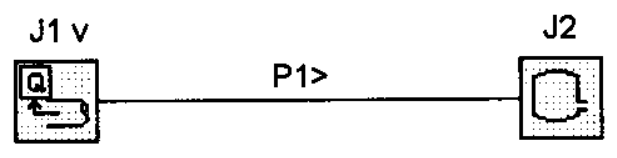

Copyright (C) 1999 Applied Flow Technology Corp. All rights reserved.

* Printed with permission. 
SNF-5331, Rev. 0

AFT Arrow 2.0 Input

Numatec Hanford Corporation

Verify21,aro - Air flow in a pipe, Example B8.7 from Nayyar Page B.343-344

Mohinder L. Nayyar

Piping Handbook, Sixth Edition, McGraw-Hill, New York, 1992

Page B.343-344, Example B8.7

Air flow - Example assumes air is a perfect gas and adiabatic flow

See Verify21.doc MS Word file for comparison with published results

Title: Verify21.aro - Air flow in a pipe, Example B8.7 from Nayyar Page B.343-344

Number Of Pipes $=1$

Number Of Junctions $=2$

Length March Solution Method with Mach Number Limits

Segments Per Pipe $=10$

Mach Number Increment $=.01$

Pressure Tolerance $=.0001$ relative change

Mass Flow Rate Tolerance $=.0001$ relative change

Enthalpy Tolerance $=.0001$ relative change

Flow Relaxation $=.5$

Pressure Relaxation $=.5$

Fluid Database: AFT Standard

Fluid= Air

Max Fluid Temperature Data $=1000$ deg. $\mathrm{K}$

Min Fluid Temperature Data $=200$ deg. $\mathrm{K}$

Molecular Weight $=28.97$ amu

Gas Constant $=0.0685486 \mathrm{Btu} / \mathrm{bm}-\mathrm{R}$

Critical Pressure $=37.25 \mathrm{~atm}$

Critical Temperature $=132.41 \mathrm{deg} . \mathrm{K}$

Acentric Factor $=.021$

Equation of State $=$ Ideal Gas

Enthalpy Model $=$ Reference

Specific Heat Ratio Accuracy $=$ High

Atmospheric Pressure $=1 \mathrm{~atm}$

Gravitational Acceleration= $1 \mathrm{~g}$ 's

Standard Pressure $=14.696 \mathrm{psia}$

Standard Temperature $=60$ deg. $F$

Turbulent Flow Above Reynolds Number $=4000$

Laminar Flow Below Reynolds Number $=2300$

Specific Heat Ratio Accuracy $=$ High

Atmospheric Pressure $=1 \mathrm{~atm}$

Gravitational Acceleration $=1 \mathrm{~g}$

Standard Pressure $=14.696 \mathrm{psia}$

Standard Temperature $=60$ deg. $F$

Turbulent Flow Above Reynolds Number $=4000$

Laminar Flow Below Reynolds Number $=2300$

Pipe Input Table

\begin{tabular}{|c|r|r|r|r|r|r|r|r|r|r|}
\hline Pipe & Name & $\begin{array}{c}\text { Pipe } \\
\text { Defined }\end{array}$ & Length & $\begin{array}{c}\text { Length } \\
\text { Units }\end{array}$ & $\begin{array}{c}\text { Hydraulic } \\
\text { Diameter }\end{array}$ & $\begin{array}{c}\text { Hydraulic } \\
\text { Diam. Units }\end{array}$ & Roughness & $\begin{array}{r}\text { Roughness } \\
\text { Units }\end{array}$ & Losses (K) & Initial Flow \\
\hline 1 & Pipe & Yes & 90 & feet & 7.981 & inches & 0.014 & Explicit $f$ & 0 & \\
\hline
\end{tabular}

\begin{tabular}{|c|c|r|c|c|c|c|c|}
\hline Pipe & $\begin{array}{c}\text { Initial Flow } \\
\text { Units }\end{array}$ & $\begin{array}{c}\text { Junctions } \\
\text { (Up,Down) }\end{array}$ & Geometry & Material & Size & Type & $\begin{array}{c}\text { Special } \\
\text { Condition }\end{array}$ \\
\hline 1 & & 1,2 & Cylindrical Pipe & Steel & 8 inch & schedule 40 & None \\
\hline
\end{tabular}

Assigned Flow Table 
SNF-5331, Rov. 0

AFT Arrow 2.0 input

Verify21.aro - Air flow in a pipe, Example B8.7 from Nayyar Page B.343-344

\begin{tabular}{|c|c|c|c|c|c|c|c|c|c|c|}
\hline Assigned Flow & Name & $\begin{array}{l}\text { Object } \\
\text { Defined }\end{array}$ & $\begin{array}{l}\text { Database } \\
\text { Source }\end{array}$ & $\begin{array}{l}\text { Special } \\
\text { Condition }\end{array}$ & Fluid & Type & Flow & $\begin{array}{l}\text { Flow } \\
\text { Units }\end{array}$ & Temperature & $\begin{array}{c}\text { Temperature } \\
\text { Units }\end{array}$ \\
\hline 1 & Assigned Flow & Yes & & None & Air & Inflow & 72000 & $\mathrm{lbm} / \mathrm{hr}$ & 101 & deg. $F$ \\
\hline
\end{tabular}

\begin{tabular}{|c|r|}
\hline Assigned Flow & $\begin{array}{c}\text { Loss } \\
\text { Model }\end{array}$ \\
\hline 1 & 0 \\
\hline
\end{tabular}

Tank Table

\begin{tabular}{|c|r|r|r|r|r|r|r|r|r|c|}
\hline Tank & Name & $\begin{array}{c}\text { Object } \\
\text { Defined }\end{array}$ & $\begin{array}{c}\text { Database } \\
\text { Source }\end{array}$ & $\begin{array}{c}\text { Special } \\
\text { Condition }\end{array}$ & Fluid & Pressure & $\begin{array}{c}\text { Pressure } \\
\text { Units }\end{array}$ & $\begin{array}{c}\text { Temperature } \\
\text { Temperature } \\
\text { Units }\end{array}$ & $\begin{array}{c}\text { Balance } \\
\text { Energy }\end{array}$ \\
\hline 2 & Tank & Yes & & N/A & Air & 14.7 & psia & 120 & deg. F & No \\
\hline
\end{tabular}

\begin{tabular}{|c|c|c|c|c|c|c|c|c|c|}
\hline Tank & $\begin{array}{c}\text { Balance } \\
\text { Concentration }\end{array}$ & $\begin{array}{c}\text { (Pipe \#1) } \\
K \ln , \text { K Out }\end{array}$ & $\begin{array}{c}\text { (Pipe \$2) } \\
\text { KIn, K Out }\end{array}$ & $\begin{array}{c}\text { (Pipe *3) } \\
\text { KIn, K Out }\end{array}$ & $\begin{array}{c}\text { (Pipe \#4) } \\
\text { K In, K Out }\end{array}$ & $\begin{array}{c}\text { (Pipe \#5) } \\
\text { K In, K Out }\end{array}$ & $\begin{array}{c}\text { (Pipe \#6) } \\
\text { KIn, K Out }\end{array}$ & $\begin{array}{c}\text { (Pipe \#7) } \\
\text { KIn, K Out }\end{array}$ & $\begin{array}{l}\text { (Pipe \#8) } \\
\text { KIn, K Out }\end{array}$ \\
\hline 2 & No & (1) 1,1 & & & & & & & \\
\hline
\end{tabular}

\begin{tabular}{|c|c|c|}
\hline Tank & $\begin{array}{c}\text { (Pipe \#9) } \\
K \ln , \text { KOut }\end{array}$ & $\begin{array}{c}\text { (Pipe } \# 10) \\
K \ln , K \text { Out }\end{array}$ \\
\hline 2 & & \\
\hline
\end{tabular}


Mohinder L. Nayyar

Piping Handbook, Sixth Edition, McGraw-Hill, New York, 1992

Page B.343-344, Example B8.7

Air flow - Example assumes air is a perfect gas and adiabatic flow

See Verify21.doc MS Word file for comparison with publiahed results

Title: Verify21 aro - Air flow in a pipe, Example B8.7 from Nayyar Page B.343-344

Analysis run on: 12/7/99 12:41:15 PM

Input File: C:LFT ProductsLAFT Arowiverificationlverify21.aro

Execution Time $=0.99$ seconds

Total Number Of Pressure literations $=0$

Total Number Of Flow Iterations= 2

Total Number Of Enthalpy Iterations= 2

Number Of Pipes $=1$

Number Of Junctions $=2$

Length March Solution Method with Mach Number Limits

Segments Per Pipe $=10$

Mach Number increment= 01

Pressure Tolerance .0001 relative change

Mass Flow Rate Tolerance= .0001 relative change

Enthalpy Tolerance= .0001 relative change

Flow Relaxation $=.5$

Pressure Relaxation $=.5$

Fluid Database: AFT Standard

Fluid= Air

Max Fluid Temperature Data $=1000$ deg. $K$

Min Fluid Temperature Data $=200$ deg. K

Molecular Weight $=28.97 \mathrm{amu}$

Gas Constant $=0.0685486$ Btu/bm-R

Critical Pressure $=37.25 \mathrm{~atm}$

Critical Temperature $=132.41 \mathrm{deg} . \mathrm{K}$

Acentric Factor $=.021$

Equation of State $=$ Ideal Gas

Enthalpy Model= Reference

Specific Heat Ratio Accuracy= High

Atmospheric Pressure= $1 \mathrm{~atm}$

Gravitational Acceleration= $1 \mathrm{~g} / \mathrm{s}$

Standard Pressure $=14.696$ psia

Standard Temperature $=60 \mathrm{deg} . \mathrm{F}$

Turbulent Flow Above Reynolds Number $=4000$

Laminar Flow Below Reynolds Number= 2300

Specific Heat Ratio Accuracy= High

Atmospheric Pressure= 1 atm

Gravitational Acceleration= $1 \mathrm{~g}$

Standard Pressure= 14.696 psia

Standard Temperature $=60 \mathrm{deg} . F$

Turbulent Flow Above Reynolds Number= 4000

Laminar Flow Below Reynolds Number= 2300

Overall Delta Pressure $=-13.3543$ psia

Total Inflow= $20.0000 \mathrm{lbm} / \mathrm{sec}$

Total Outflow= $20.0000 \mathrm{lbm} / \mathrm{sec}$

Total Energy Inflow= 4149.09 Btu/s

Total Energy Outflow= 4149.09 Btu/s

Total Heat Transferred into System $=0.00000 \mathrm{Btu} / \mathrm{s}$

Maximum Pressure is 24.9467 psia at Junction 1 Inlet

Minimum Pressure is 14.7000 psia at Junction 2 Outlet

Maximum Static Temperature is $120.000 \mathrm{deg}$. F at Junction 2 Inlet

Minimum Static Temperature is $119.834 \mathrm{deg}$. F at Junction 1 Inlet

\section{Pipe Output Table}


SNF-5331, Rev. 0

AFT Arrow 2.0 Output

(2)

$12 \pi / 99$

Verify21.aro - Air flow in a pipe, Example B8.7 from Nayyar Page B.343-344

\begin{tabular}{|c|c|c|c|c|c|c|c|c|c|c|c|}
\hline Pipe & $\begin{array}{l}\text { Mass } \\
\text { Flow } \\
\text { (lbm/hr) }\end{array}$ & $\begin{array}{c}\text { Vel. In } \\
\text { (feet/sec) }\end{array}$ & $\begin{array}{l}\text { Vel. Out } \\
\text { (feet/sec) }\end{array}$ & $\begin{array}{c}\text { Mach } \\
\text { in }\end{array}$ & $\begin{array}{l}\text { Mach } \\
\text { \# Out }\end{array}$ & $\begin{array}{c}\text { P Static } \\
\text { In } \\
\text { (psia) }\end{array}$ & $\begin{array}{c}\text { P Static } \\
\text { Out } \\
\text { (psia) }\end{array}$ & $\begin{array}{c}\text { T Static } \\
\text { In } \\
\text { (deg. F) }\end{array}$ & $\begin{array}{c}\text { T Static } \\
\text { Out } \\
\text { (deg. F) }\end{array}$ & $\begin{array}{c}\text { T Stag. } \\
\text { In } \\
\text { (deg. F) }\end{array}$ & $\begin{array}{c}\text { T Stag. } \\
\text { Out } \\
\text { (deg. F) }\end{array}$ \\
\hline 1 & $72,000,0$ & 479.282 & 800.696 & 0.414173 & 0.713575 & 24.9468 & 14.0338 & 101.000 & 67.2485 & 119.834 & 119.877 \\
\hline
\end{tabular}

All Junction Table

\begin{tabular}{|c|c|c|c|c|}
\hline Jct & $\begin{array}{c}\text { P Static } \\
\text { In } \\
\text { (poia) }\end{array}$ & $\begin{array}{c}\text { P Static } \\
\text { Out } \\
\text { (psia) }\end{array}$ & $\begin{array}{c}\text { T Static } \\
\text { In } \\
\text { (deg. F) }\end{array}$ & $\begin{array}{c}\text { T Static } \\
\text { Out } \\
\text { (deg. F) }\end{array}$ \\
\hline 1 & 24.9467 & 24.9467 & 101.000 & 101.000 \\
\hline 2 & 19.6747 & 14.7000 & 120.000 & 120.000 \\
\hline
\end{tabular}




\section{DISTRIBUTION SHEET}

To

Distribution

Project TitleMork Order

SNF-5331
From

FDH - Engineering Labs
Page 1 of 1

Date December 1999

EDT No. 627034

ECN No. N/A

\section{Name}

B. A. Crea

T. Choho

F. J. Heard (4)

J. J. Irwin

C. R. Miska

S. L. Mischke

M. J. Schliebe

J. R. Brehm

Central Files

DIMC

DOE/RL Reading Room

SNF Project File W-441

CVD Library

Startup Library

CVD Satellite Library

\begin{tabular}{|c|c|c|c|c|}
\hline MSIN & $\begin{array}{l}\text { Text } \\
\text { With All } \\
\text { Attach. }\end{array}$ & Text Only & $\begin{array}{l}\text { Attach / } \\
\text { Appendix } \\
\text { Only }\end{array}$ & $\begin{array}{c}\text { EDT/ECN } \\
\text { Only }\end{array}$ \\
\hline R3-86 & $\mathrm{x}$ & & & \\
\hline R3-86 & $\mathrm{x}$ & & & \\
\hline $66-3590-34$ & $x$ & & & \\
\hline R3-86 & $x$ & & & \\
\hline R3-86 & $\mathrm{x}$ & & & \\
\hline R3-86 & $x$ & & & \\
\hline L6-13 & $\mathbf{x}$ & & & \\
\hline R3-26 & $x$ & & & \\
\hline B1-07 & $x$ & & & \\
\hline H6-15 & $x$ & & & \\
\hline H2-53 & $x$ & & & \\
\hline R3-11 & $x$ & & & \\
\hline R3-86 & $x$ & & & \\
\hline B2-64 & $x$ & & & \\
\hline$x 3-25$ & $\mathrm{x}$ & & & \\
\hline
\end{tabular}

